\title{
Promedios Esféricos, Análisis Espectral y la Ecuación de Weyl
}

\author{
Tesis \\ que para obtener el grado de Doctor en Ciencias presenta
}

Jesús Chargoy Corona

bajo la dirección del Dr. Antoni Wawrzyńczyk

Departamento de Matemáticas

Universidad Autónoma Metropolitana-Iztapalapa 


\section{Introducción}

El método de separación de variables y sus variantes es uno de los métodos que tiene gran trascendencia en la resolución de ecuaciones diferenciales en derivadas parciales (e.d.d.p.). Como se sabe, las primeras ideas se deben a Leonhard Euler que las desarrolló al resolver la ecuación de onda en dos dimensiones

$$
\begin{gathered}
\frac{\partial^{2} u}{\partial t^{2}}(x, t)=c^{2} \frac{\partial^{2} u}{\partial x^{2}} ; \quad c>0, t \in \mathbb{R}, x \in \mathbb{R} \\
u(x, 0)=f(x), \quad \frac{\partial u}{\partial t}(x, 0)=g(x) .
\end{gathered}
$$

Hablando de manera informal, (para el caso de dimensión $n$ ) la idea esencial de este método consiste en hallar coordenadas adecuadas que nos permitan descomponer el dominio de la solución como un producto de dos variedades transversales $M$ y $N$ de manera tal que la ecuación se divida en dos ecuaciones "independientes"; una para $M$. y otra para $N$. Las soluciones de tales ecuaciones dependen en general de parámetros discretos o continuos y el problema de hallar la solución de la ecuación original se reduce a expresarla como límite de combinaciones lineales de productos de las primeras.

Ya que también diferentes tipos de integrales (Bochner, Riemann-Stieltjes, Lebesgue) pueden interpretarse como límite de sumas, dicha reducción nos conduce de manera natural a tres problemas:

i) Representación de una función como serie convergente de funciones elementales (p.e., las series de Fourier).

ii) Representación de una función como integral con respecto a una medida que satisfaga ciertas propiedades reproductoras (p.e., núcleos integrales).

iii) Preservación de la regularidad de estas representaciones (p.e., $C^{k}, C^{\infty}$, analiticidad, etc.).

En casos concretos como la ecuación de Laplace y la ecuación de onda tales ideas se desarrollan de manera elemental, detallada y rigurosa en [12] y [15].

Otros puntos de vista más técnicos y generales que incluyen la teoría de distribuciones pueden encontrarse en [7], [8], [11] y [16]. 
Si interpretamos la integral como un promedio de funciones con un peso adecuado, entonces las soluciones de las ecuaciones antes mencionadas poseen un elemento en común: un promedio esférico adecuado.

Para precisar lo anterior, escribamos $S^{n-1}=\left\{x \in \mathbb{R}^{n} \mid\|x\|=1\right\}$ y denotemos por $d s_{n-1}$ el elemento de superficie normalizado en la esfera $S^{n-1}$. En particular $S^{0}=\{1,-1\}$ y $d s_{0}( \pm 1)=1 / 2$.

Dada una función continua $f: \mathbb{R}^{n} \rightarrow \mathbb{R}$, definimos el promedio esférico de $f$ alrededor de $x \in \mathbb{R}^{n}$ en la esfera $S_{r}^{n-1}(x)=\left\{y \in \mathbb{R}^{n} \mid\|y-x\|=r\right\}$ por la fórmula

$$
M_{f}(x, r)=P\left(\tau_{-x} f\right)(r)=\int_{S^{n-1}} f(x+r \xi) d s_{n-1}(\xi),
$$

donde $\left(\tau_{-x} f\right)(y)=f(x+y), y \in \mathbb{R}^{n}$. Mediante un cálculo elemental, para $f \in C^{2}\left(\mathbb{R}^{n}\right)$ obtenemos

$$
\left(\frac{\partial^{2} M_{f}}{\partial r^{2}}+\frac{(n-1)}{r} \frac{\partial M_{f}}{\partial r}\right)(x, r)=\Delta_{x} M_{f}(x, r)=M_{\Delta f}(x, r)
$$

De aquí se concluye: $\Delta f=0 \Leftrightarrow M_{f}(x, r)=f(x), \quad r \in \mathbb{R}^{+}$.

Ahora supóngase que $\Omega \subset \mathbb{R}^{n}$ es abierto y $\Omega$ contiene la cerradura de la bola $B_{r}=\left\{y \in \mathbb{R}^{n} \mid\|y\|<r\right\}$. Si $f \in C^{2}(\Omega)$, entonces por el Teorema de Stokes

$$
\begin{aligned}
f(x) & =\frac{1}{(2-n)} \int_{0}^{r} t^{n-1} \int_{S^{n-1}} \frac{\Delta f(t \xi) d s_{n-1}(\xi)}{\|x-t \xi\|^{n-2}}- \\
& -\frac{r^{n-1}}{2-n} \int_{S^{n-1}}\|x-r \xi\|^{2-n} \frac{\partial f(r \xi)}{\partial r} d s_{n-1}(\xi) \\
& +\frac{r^{n}}{(2-n)} \int_{S^{n-1}}\|x-r \xi\|^{-n} f(r \xi) d s_{n-1}(\xi) .
\end{aligned}
$$

Las integrales sobre $S^{n-1}$ que aparecen aquí no son del tipo $M_{f}$, sin embargo, éstas pueden reinterpretarse como "promedios esféricos adecuados".

A). En la ecuación de onda

$$
\begin{gathered}
\frac{\partial^{2} u(x, t)}{\partial t^{2}}=C^{2} \Delta_{x} U(x, t) ; t \geq 0, x \in \mathbb{R}^{n} \\
u(x, 0)=g(x), \frac{\partial u}{\partial t}(x, 0)=f(x)
\end{gathered}
$$


donde $c$ es una constante positiva, se definen los promedios esféricos por la fórmula

$$
M_{u}(x, r, t)=\int_{S^{n-1}} u(x+r \xi, t) d s_{n-1}(\xi)
$$

y se cumple $u(x, t)=M_{u}(x, 0, t)$. Si $u$ es solución de esta ecuación entonces

$$
M_{u}(x, r, 0)=M_{g}(x, r), \frac{\partial M_{u}}{\partial t}(x, r, 0)=M_{f}(x, r)
$$

y

$$
\frac{\partial^{2} M_{u}}{\partial t^{2}}(x, r, t)=c^{2}\left(\frac{\partial^{2} M_{u}}{\partial r^{2}}+\frac{(n-1)}{r} \frac{\partial M_{u}}{\partial r}\right)(x, r, t) .
$$

Hasta aquí hemos bosquejado la manera en que los promedios esféricos reducen el problema original. También hemos visto que las representaciones integrales esféricas o "promedios esféricos adecuados" reproducen funciones regulares a partir de sus valores en esferas. Tales representaciones aparecen en otras ramas de la Matemática:

B). en Análisis Complejo:

Si $\Omega \subset \mathbb{C}^{n}$ es abierto y $f$ es analítica en $\Omega$ entonces:

(a) Para $n=1$ tenemos la Fórmula Integral de Cauchy

$$
f(z)=\frac{1}{2 \pi i} \int_{\left|\lambda-z_{0}\right|=} \frac{f(\lambda) d \lambda}{\lambda-z}=\frac{r}{2 \pi} \int_{S^{1}} \frac{f\left(z_{0}+r \xi\right) \xi d s(\xi)}{z_{0}-z+r \xi}
$$

válida cuando $\left\{\left|\omega-z_{0}\right| \leq r\right\} \subset \Omega$ y $\left|z-z_{0}\right|<r$.

(b) Para $n \geq 2$, si $f$ es analítica en la bola cerrada unitaria,

$$
f(z)=\frac{(n-1) !}{2 \pi^{n}} \int_{S^{2 n-1}} \frac{\left(1-\|z\|^{2}\right)^{n} f(\xi)}{\|1-\langle z, \xi)\|^{2 n}} d s_{n-1}(\xi), \quad\|z\|<1 .
$$

\section{C). En la Transformada de Radón:}

Dado $(\omega, t) \in S^{n-1} \times \mathbb{R}$, la ecuación $\langle y, \omega\rangle=t$ define un hiperplano en $\mathbb{R}^{n}$. Recíprocamente, si $\xi \subset \mathbb{R}^{n}$ es un hiperplano, entonces dados $x \in \xi$ y $\omega \in S^{n-1}$ ortogonal a $\xi$ obtenemos $\xi=\left\{y \in \mathbb{R}^{n}:\langle y, \omega\rangle=\langle x, \omega\rangle\right\} . \mathbb{Z}_{2}=\{-1,1\}$ actúa en $S^{n-1} \times \mathbb{R}$ por multiplicación y se tiene que $\left(S^{n-1} \times \mathbb{R}\right) / \mathbb{Z}_{2}=\mathbb{P}^{n}$ es la 
colección de todos los hiperplanos en $\mathbb{R}^{n}$. Mediante la proyección canónica, $\mathbb{P}^{n}$ adquiere una estructura de variedad suave:

$$
\left.\right|_{S^{n-1} \times \mathbb{R}^{\mathbb{Z}_{2}}} ^{\mid \pi(\omega, t):=[\omega, t]}
$$

En particular, la colección de planos que pasan por un punto $x \in \mathbb{R}^{n}$ queda definida como el conjunto compacto $\left.\left\{[\omega,\langle x, \omega\rangle] \in \mathbb{P}^{n}\right]: \omega \in S^{n-1}\right\}$.

Si $f \in C^{0}\left(\mathbb{R}^{n}\right)$ es integrable en cada hiperplano, entonces definimos la transformada de Radón de $f$ por la fórmula

$$
\Re(f)(\{\omega, t])=\int_{\{\langle\omega, x\rangle=t\}} f(x) d m(x) ;[\omega, t] \in \mathbb{P}^{n},
$$

donde $d m$ es la medida de Lebesgue en el hiperplano $\{\langle\omega, x\rangle=t\}$. Se sigue que $\Re(f) \in C^{0}\left(\mathbb{P}^{n}\right)$.

Se define la transformada dual de Radón de $\phi \in C^{0}\left(\mathbb{P}^{n}\right)$ por la relación

$$
\Re^{\vee}(\phi)(x)=\int_{S^{n-1}} \phi(|\omega,\langle x, \omega\rangle|) d s_{n-1}(\omega) ; x \in \mathbb{R}^{n} .
$$

si $f \in C^{2}\left(\mathbb{R}^{n}\right)$, entonces

$$
\left.\Re(\Delta f)([x, t])=\frac{\partial^{2}}{\partial t^{2}} \Re(f)(\mid x, t]\right) .
$$

La relación entre estas transformadas viene dada por el Teorema de Inversión:

Si $f$ es rápidamente decreciente en $\mathbb{R}^{n}$, entonces

$$
c f=\Delta^{\frac{n-1}{2}} \Re^{\vee}(\Re f),
$$

donde $c$ es una constante que no depende de $f$.

La justificación de $\Delta^{p}$ se encuentra en $[9,(a) \S 8]$ y [11, cap. III sección 2].

La razón por la cual el empleo de coordenadas y prómedios esféricos reduce estos problemas a funciones radiales es la invariancka bajo rotaciones 
del operador diferencial de Laplace, i.e., si $k$ es la matriz asociada a una rotación de ejes y si $y=k \cdot x, x \in \mathbb{R}^{n}$, entonces

$$
\sum_{j=1}^{n} \frac{\partial^{2}}{\partial y_{j}^{2}}=\sum_{j=1}^{n} \frac{\partial^{2}}{\partial x_{j}^{2}}
$$

Como se sabe, la colección de rotaciones forma un grupo compacto $K$; a saber:

$$
K=\left\{\text { matrices } k n \times n \mid k \cdot{ }^{t} k=I\right\},
$$

donde ${ }^{t} k$ denota la transpuesta de $k$.

De manera natural, cada $k \in K$ define un operador $L_{k}$ actuando en funciones:

$$
\left(L_{k} f\right)(x)=f\left(k^{-1} \cdot x\right) .
$$

- Si denotamos por $\delta$ la medida de Dirac concentrada en el origen, entonces las ecuaciones de Laplace y de Onda pueden reescribirse como

$$
(\Delta \delta) * f=0
$$

y

$$
\left(\frac{\partial^{2}}{\partial t^{2}}-c^{2} \Delta \delta *\right) u=0
$$

De este modo ambos problemas quedan comprendidos en uno más general:

iv) Describir el espacio solución de un sistema de ecuaciones

$$
T * f=0 ; \quad T \in \mathcal{A},
$$

donde $\mathcal{A}$ es una familia de distribuciones invariante bajo rotaciones.

Sea $V$ tal espacio entonces éste es un espacio lineal invariante bajo rotaciones y traslaciones.

Otra de las técnicas ampliamente utilizadas en la Teoría de e.d.d.p. es la representación de un operador autoadjunto como una integral con respecto a una familia espectral.

Como consecuencia de esta teoría espectral, se deduce que la familia de operadores

$$
e^{t \alpha \cdot \nabla}: L^{2}\left(\mathbb{R}^{n}\right)^{N} \rightarrow L^{2}\left(\mathbb{R}^{n}\right)^{N}, t \geq 0, \quad
$$


es un semigrupo de operadores unitarios que satisface la ecuación diferencial matricial de Weyl

$$
\begin{gathered}
\frac{\partial \psi}{\partial t}(t, x)=\sum_{j=1}^{n} \alpha_{j} \frac{\partial \psi}{\partial x_{j}}(t, x) ; t>0, x \in \mathbb{R}^{n} \\
\psi(0, x)=\phi(x) \in \text { Dominio }(\alpha \cdot \nabla),
\end{gathered}
$$

donde $\alpha_{1}, \ldots, \alpha_{n}$ son matrices complejas $N \times N$ con la propiedad

$$
\alpha_{j} \alpha_{k}+\alpha_{k} \alpha_{j}=2 \delta_{j k} .
$$

Esta e.d.d.p. matricial no es invariante bajo rotaciones; sin embargo, el operador diferencial de Weyl

$$
\frac{\partial}{\partial t}-\sum_{j=1}^{n} \alpha_{j} \frac{\partial}{\partial x_{j}}=\frac{\partial}{\partial t}-\alpha \cdot \nabla
$$

es una raíz de la ecuación de onda matricial, i.e.,

$$
\left(\frac{\partial}{\partial t}-\alpha \cdot \nabla\right)^{2}=\frac{\partial^{2}}{\partial t^{2}}-\Delta
$$

De este hecho se sigue que el núcleo o kernel del operador diferencial de Weyl es invariante bajo rotaciones y translaciones y, por lo tanto, su estudio también se reduce al problema iv). 


\section{Notación y Preliminares}

El objetivo de este capítulo es decribir de manera breve los resultados obtenidos en este trabajo así como la relación entre éstos y la que se deriva como analogía o extensión de otros ya conocidos.

I.1. El Espacio $\mathcal{E}\left(\mathbb{R}^{n}\right)$ : Sea $n \in \mathbb{N}$ fijo. Dada una sucesión de compactos $K_{\ell} \subset \mathbb{R}^{n}$ con la propiedad $K_{\ell} \subset \dot{K}_{\ell+1}, \ell=1,2, \ldots$, definimos la sucesión creciente de seminormas

$$
\|f\|_{\ell}=\sup _{\substack{x \in k_{\ell} \\ \alpha_{1}+\cdots+\alpha_{n} \leq \ell}}\left\|\frac{\partial^{\alpha_{1}+\ldots+\alpha_{n}} f(x)}{\partial x_{1}^{\alpha_{1}} \cdots \partial x_{n}^{\alpha_{n}}}\right\| ; f \in C^{\infty}\left(\mathbb{R}^{n}\right) .
$$

La métrica asociada viene dada por

$$
d(f, g)=\sum_{\ell=1}^{+\infty} 2^{-\ell} \frac{\|f-g\|_{\ell}}{1+\|f-g\|_{\ell}}, f, g \in C^{\infty}\left(\mathbb{R}^{n}\right) .
$$

Así $C^{\infty}\left(\dot{\mathbb{R}}^{n}\right)$ adquiere una estructura de espacio métrico y la topología inducida no depende de la sucesión de compactos $\left\{K_{\ell}\right\}$. De este modo podemos suponer que $K_{\ell}$ es la bola de radio $\ell \in \mathbb{N}$ centrada en el origen. El espacio métrico así construido será denotado por $\mathcal{E}\left(\mathbb{R}^{n}\right)$ y tiene las siguientes propiedades

(a) $\mathcal{E}\left(\mathbb{R}^{n}\right)$ es completo y localmente convexo.

(b) El conjunto de funciones suaves con soporte compacto $C_{0}^{\infty}\left(\mathbb{R}^{n}\right)$ es denso en $\mathcal{E}\left(\mathbb{R}^{n}\right)$.

(c) Una familia $\mathcal{A} \subset \mathcal{E}\left(\mathbb{R}^{n}\right)$ es un subconjunto compacto $\Leftrightarrow \mathcal{A}$ es cerrado y acotado.

Es decir $\mathcal{E}\left(\mathbb{R}^{n}\right)$ es un espacio de Fréchet con la propiedad de Heine-Borel, (ver [19, (b)). En ([5, Cap. V]) se demuestra que el dual topológico de $\mathcal{E}\left(\mathbb{R}^{n}\right)$, $\mathcal{E}^{\prime}\left(\mathbb{R}^{n}\right)$, consta de todas las distribuciones con soporte compacto contenido en $\mathbb{R}^{n}$. Dotando a $\mathcal{E}^{\prime}\left(\mathbb{R}^{n}\right)$ con la topología inducida por las seminormas

$$
\|T\|_{\mathcal{A}}:=\sup _{f \in \mathcal{A}}\|T f\|, T \in \mathcal{E}^{\prime}\left(\mathbb{R}^{n}\right) ; \mathcal{A} \subset \mathcal{E}\left(\mathbb{R}^{n}\right) \text { acotado }
$$


se verifica que $\mathcal{E}\left(\mathbb{R}^{n}\right)$ y $\mathcal{E}^{\prime}\left(\mathbb{R}^{n}\right)$ son espacios reflexivos. Si $x \in \mathbb{R}^{n}, f \in \mathcal{E}\left(\mathbb{R}^{n}\right)$ y $T \in \mathcal{E}^{\prime}\left(\mathbb{R}^{n}\right)$ definimos

$$
\begin{gathered}
\left(\tau_{x} f\right)(y):=f(y-x),\left(\tau_{x} T\right)(f)=T\left(\tau_{-x} f\right), \\
\check{f}(y):=f(-y), \quad \check{T}(f)=T(\check{f}) .
\end{gathered}
$$

La convolución se establece por las fórmulas

$$
(T * f)(x)=T\left(\tau_{x} \check{f}\right) \text { y }\left(T_{1} * T_{2}\right)(f)=T_{1}\left(\breve{T}_{2} * f\right), T_{1}, T_{2} \in \mathcal{E}^{\prime}\left(\mathbb{R}^{n}\right) .
$$

$\left(\mathcal{E}^{\prime}\left(\mathbb{R}^{n}\right), *\right)$ es una $\mathbb{C}$-álgebra conmutativa cuya unidad es la medida de Dirac concentrada en el origen. Así, $\mathcal{E}\left(\mathbb{R}^{n}\right)$ es un $\left(\mathcal{E}^{\prime}\left(\mathbb{R}^{n}\right), *\right)$-módulo.

Para $z=\left(z_{1}, \ldots, z_{n}\right) \in \mathbb{C}^{n}$ y $T \in \mathcal{E}^{\prime}\left(\mathbb{R}^{n}\right)$ defínanse $e_{z}\left(x_{1}, \ldots, x_{n}\right)=$ $\exp \left[i\left(z_{1} x_{1}+z_{2} x_{2}+\cdots+z_{n} x_{n}\right)\right]$ y $\hat{T}(\mathfrak{z})=T\left(e_{-z}\right)$. Sea $\mathcal{P W}\left(\mathbb{C}^{n}\right)$ el subespacio de funciones enteras en $\mathbb{C}^{n}$ para las cuales hay constantes $A, B, C \geq 0$ que satisfacen

$$
|f(\mathfrak{z})| \leq A(1+\|z\|)^{B} e^{c \|} \operatorname{Im} \xi \|, z \in \mathbb{C}^{n} .
$$

El célebre Teorema de Paley-Wiener-Schwartz asegura que la transformada de Fourier ${ }^{\wedge}: \mathcal{E}^{\prime}\left(\mathbb{R}^{n}\right) \rightarrow \mathcal{P W}\left(\mathbb{C}^{n}\right), T \mapsto \hat{T}$ es un isomorfismo de álgebras.

I.2. Una función $f \in \mathcal{E}\left(\mathbb{R}^{n}\right)$ se dice periódica en promedio si se cumple

$$
T * \check{f}=0 \text { para algún } 0 \neq T \in \mathcal{E}^{\prime}\left(\mathbb{R}^{n}\right) \text {. }
$$

Dados $V \subset \mathcal{E}\left(\mathbb{R}^{n}\right)$ y $\mathfrak{a} \subset \mathcal{E}^{\prime}\left(\mathbb{R}^{n}\right)$ se definen sus anuladores por las relaciones

$$
\begin{aligned}
& V^{\perp}=\left\{T \in \mathcal{E}^{\prime}\left(\mathbb{R}^{n}\right): T * \check{f}=0\right\} \subset \mathcal{E}^{\prime}\left(\mathbb{R}^{n}\right) \\
& \mathfrak{a}^{\perp}=\left\{\phi \in \mathcal{E}\left(\mathbb{R}^{n}\right): T * \dot{\phi}=0\right\} \subset \mathcal{E}\left(\mathbb{R}^{n}\right) .
\end{aligned}
$$

Es claro que $V^{\perp}$ es un ideal cerrado de $\mathcal{E}^{\prime}\left(\mathbb{R}^{n}\right)$ y a ${ }^{\perp}$ es un subespacio lineal cerrado invariante bajo translaciones en $\mathcal{E}\left(\mathbb{R}^{n}\right)$.

Si $V$ es un subespacio lineal cerrado e invariante bajo translaciones, entonces se define el espectro de $V^{\perp}$ como el conjunto

$$
\sigma\left(V^{\perp}\right):=\left\{z \in \mathbb{C}^{n}: \hat{T}(z)=0, T \in V^{\perp}\right\}
$$

En el caso $n=1$, L. Schwartz mostró $[20$-(a)] que

(a) $0 \neq V \neq \mathcal{E}(\mathbb{R}) \Rightarrow \sigma\left(V^{\perp}\right) \neq \phi$ 
(b) Si $\lambda \in \sigma\left(V^{\perp}\right)$ y

$$
m(\lambda)=\max \left\{m \in \mathbb{Z} \mid \hat{T}^{(j)}(\lambda)=0 ; 0 \leq j \leq m, T \in V^{\perp}\right\},
$$

entonces para cada $f \in V$ hay una partición $\Omega$ de la variedad de ceros $\left\{\sigma\left(V^{\perp}\right), m(\lambda)\right\}$ de manera tal que $f$ admite una representación $\mathcal{E}(\mathbb{R})$-convergente

$$
f(x)=\sum_{\Omega} a_{\lambda, j} x^{j} e^{-i \lambda} x
$$

En el caso general $n \geq 2$ Gurevich [Gu- ] exhibe un ideal a cuyo espectro es vacío.

Los incisos (a) y (b) son conocidos como análisis y síntesis espectral respectivamente.

Denotemos por $M(n)$ el grupo de movimientos rígidos en $\mathbb{R}^{n}$ y considérese el problema de obtener análisis y síntesis espectral para un subespacio cerrado $0 \neq V \subsetneq \mathcal{E}\left(\mathbb{R}^{n}\right) M(n)$-invariante. La idea esencial es la reducción de este problema al caso conocido $n=1$ resuelto por L. Schwartz.

En [BST ] y [BZ ] emplean la parte (a) del teorema de Schwartz para demostrar que $V$ contiene funciones exponenciales $e_{-z}$, i.e., $\sigma\left(V^{\perp}\right)$ no es vacío.

Aplicando el teorema 7.3.6 de [Hör ], la síntesis espectral se obtiene al demostrar que $V$ es la cerradura del espacio lineal generado por las funciones polinomio-exponenciales

$$
P(x) e^{-i(3, x)}, \quad P(x) \text { un polinomio, }
$$

contenidas en $V$.

En [Wawr ] A. Wawrzyńczyk emplea el teorema de Schwartz para obtener análisis y síntesis espectral en el espacio de horociclos.

Para obtener síntesis espectral él emplea la representación (I.1.4) y muestra que los coeficientes dependen continuamente de las translaciones izquierdas definidas por la representación. El método que emplea para obtener análisis espectral es diferente al utilizado en [BST ] y [BZ ].

A la fecha no hay un método para determinar los polinomios exponenciales contenidos en $V$. Por esta razón en el capítulo II presentamos un método nuevo para obtener síntesis espectral en el caso del grupo $M(n)$. Aquí aplicamos el clásico teorema de Ehnrenpreis-Malgrange y los métodos 
elementales de ecuaciones diferenciales ordinarias para obtener la síntesis espectral y otros resultados.

Efectivamente, las funciones radiales contenidas en $V$ generan un subespacio lineal cerrado $W \subsetneq \mathcal{E}\left(\mathbb{R}^{n}\right)$ invariante bajo translaciones. Por el teorema de Schwartz, hay una "variedad definiente" $\left\{\sigma\left(W^{\perp}\right), m(\lambda)\right\}$ tal que los subespacios

$$
\mathcal{E}_{\lambda}^{m(\lambda)}=\left\{f \in \mathbb{R}^{n} \mid\left(\Delta+\lambda^{2}\right)^{m(\lambda)+1} f=0\right\}, \lambda \in \sigma\left(W^{\perp}\right) \subset \mathbb{C}
$$

satisfacen

$$
V=\left(\mathcal{E}_{0}^{\left.\frac{m(0)}{2}\right)} \cap V\right) \oplus \overline{\sum_{\lambda \in \sigma\left(W^{\perp}\right)} \mathcal{E}_{\lambda}^{m(\lambda)}}
$$

donde $\bar{\Sigma}$ denota la $\mathcal{E}\left(\mathbb{R}^{n}\right)$-cerradura de la suma directa algebraica de espacios vectoriales (ver II.8 y II.14).

(I.b) Para cada $\lambda \in \sigma\left(W^{\perp}\right)$ hay una distribución $L_{\lambda} \in \mathcal{E}^{\prime}\left(\mathbb{R}^{n}\right)$ y funciones $\phi_{\lambda, j}, 0 \leq j \leq m(\lambda)$, tales que el operador $q_{\lambda}: V \rightarrow \mathcal{E}_{\lambda}^{m(\lambda)}$ definido por

$$
q_{\lambda}(f)(x)=\sum_{j=0}^{m(\lambda)}\left[\left(\Delta+\lambda^{2}\right)^{j} L_{\lambda} * f\right](x) \phi_{\lambda, j}, \quad f \in V
$$

es una proyección continua, (ver II.12 y II.15).

En particular, cada $f \in V$ está determinada por sus proyecciones.

(I.c) Para cada $z \in \mathbb{C}, z \notin \sigma\left(W^{\perp}\right)$ el operador diferencial $\left.\left(\Delta+z^{2}\right)\right|_{V}$ : $V \rightarrow V$ tiene un inverso continuo $R_{z}: V \rightarrow V$, (ver II.9).

Finalmente, la aplicación

$$
C_{z}(f)=\Re_{z}(f)(0), \quad f \in V, z \notin \sigma\left(W^{\perp}\right)
$$

define un operador lineal continuo e inyectivo de $V$ en el espacio de funciones meromorfas cuyos polos están contenidos en $\sigma\left(W^{\perp}\right)$, (ver II.17).

I.3. En el capítulo III veremos que en el espacio de Hilbert $L^{2}\left(\mathbb{R}^{n}\right)^{N}$ es posible aproximar la solución a la ecuación de Weyl mediante promedios esféricos adecuados de la condición inicial. La interpretación física de tales 
promedios permite establecer las soluciones de las ecuaciones de Weyl y Dirac como integrales sobre espacios de trayectorias para el caso $n$-dimensional.

Sean $\alpha_{1}, \ldots, \alpha_{n}$ matrices complejas $N \times N$, autoadjuntas con la propiedad anticonmutativa

$$
\alpha_{j} \alpha_{k}+\alpha_{k} \alpha_{j}=2 \delta_{j k} I_{N}
$$

donde $\delta_{j k}$ es la delta de Kronecker e $I_{N}$ es la $N \times N$-matriz identidad. $\mathrm{Si}$ $\phi_{1}, \ldots, \phi_{N}: \mathbb{R}^{n} \rightarrow \mathbb{C}$ son funciones medibles, la ecuación de Weyl sin potencial con condición inicial $\phi=\left(\phi_{1}, \ldots, \phi_{N}\right)$ es la ecuación diferencial en derivadas parciales (e.d.d.p.) dada por

$$
\begin{aligned}
& \frac{\partial \psi}{\partial t}=\alpha \cdot \nabla \psi=\sum_{j=1}^{n} \alpha_{j} \frac{\partial \psi}{\partial x_{j}}, \psi=\psi(t, x), t>0 \\
& \psi(0, x)=\phi(x) .
\end{aligned}
$$

Ahora supongamos que $\phi$ es localmente integrable y definamos los "promedios esféricos adecuados" por la fórmula

$$
[T(t) \phi](x)=\int_{S^{n-1}}\left(I_{N}+\alpha \cdot \xi\right) \phi(x+n t \xi) d s_{n-1}(\xi),
$$

donde $x \in \mathbb{R}^{n}, t \in \mathbb{R}, S^{n-1} \subset \mathbb{R}^{n}$ es la $(n-1)$-esfera unitaria, $d_{S_{n-1}}$ es la medida de Lebesgue normalizada en $S^{n-1}$ y $\alpha \cdot \xi:=\sum_{j=1}^{n} \xi_{j} \alpha_{j}$.

En este capítulo también mostraremos que si $\phi \in L^{2}\left(\mathbb{R}^{n}\right)^{N}$, entonces

$$
\lim _{m \rightarrow+\infty} T(t / m)^{m} \phi=\psi(t, \cdot)
$$

donde $\psi$ satisface (I.3.2) y el límite se entiende en la topología de $L^{2}\left(\mathbb{R}^{n}\right)^{N}$.

Promedics esféricos similares e iteraciones de éstos fueron considerados en [3] para $n=3$. En nuestro trabajo lo hacemos para dimensión general $n$ e introducimos la rapidez de propagación igual a $n$ para obtener obtenemos el generador infinitesimal de Weyl. Mediante una representación estocástica aproximamos también la solución de la ecuación de Dirac mediante promedios esféricos (ver III.7 y [18]).

En este punto surgen de manera natural las siguientes,preguntas: I.4 Si $\phi$ es una función suave, ¿Se preservarán las convergenciaś de las derivadas 
de $T(t) \phi$ a las derivadas de $\psi$ ?, ¿Hay convergencia uniforme en subconjuntos compactos de $\mathbb{R}^{n}$ ?

En el capítulo IV se rèsponde afirmativamente al problema de la convergencia en la topología de $\mathcal{E}\left(\mathbb{R}^{n}\right)$ para las aproximaciones esféricas a la solución de la ecuación de Weyl en el caso de potenciales y condiciones iniciales suaves (ver IV.8).

La respuesta afirmativa a estas cuestiones también tiene interés computacional en Análisis Numérico para aproximar funciones de onda. La naturaleza esférica de las aproximaciones (I.3.3) muestra que los cálculos de tales promedios se reducen al considerar condiciones iniciales con simetría esférica. De este modo el problema computacional se reduce a encontrar familias adecuadas de funciones radiales que, como en el caso de las series de Fourier, nos permitan descomponer funciones más generales en términos de series $\mathcal{E}\left(\mathbb{R}^{n}\right)$-convergentes.

Definamos $V$ como la $\mathcal{E}\left(\mathbb{R}^{n}\right)$-cerradura del espacio lineal generado por las funciones

$$
\left(L_{k} \tau_{x} \phi\right)(y):=\phi(-x+k \cdot y) ; k \in K, x \in \mathbb{R}^{n} .
$$

El espacio $V \subset \mathcal{E}\left(\mathbb{R}^{n}\right)$ es cerrado e invariante bajo rotaciones y translaciones. Ya que el operador

$$
e^{t a \cdot \nabla}: \mathcal{E}\left(\mathbb{R}^{n}\right) \rightarrow \mathcal{E}\left(\mathbb{R}^{n}\right), \quad t \in \mathbb{R}
$$

envía funciones suaves $\phi$ en soluciones de (I.4.1) y tomando en cuenta que este operador queda definido como la convolución con una matriz de coeficientes distribucionales con soporte compacto, entonces la descripción de $V$ en el capítulo II nos da la solución al problema computacional.

En el caso suave de potenciales $V_{j}(x), j=1, \ldots, n$ o perturbaciones $B(x)$, la existencia y unicidad de las soluciones a las ecuaciones de Weyl y de Dirac queda garantizada al pedir que estos operadores matriciales de multiplicación sean simétricos o bien, real valuados y acotados (ver [11], [12] o [22]).

En el resultado que aquí obtenemos no pedimos restricción alguna y como consecuencia del método que empleamos se obtiene también la existencia y unicidad de la solución a la ecuación de Weyl aún en el caso de potencial suave no acotado.

Para garantizar la propiedad de semigrupo de una familia de operadores $\{Q(t)\}_{t \geq 0}$ definidos en un espacio de Fréchet, se requiere'que el generador infinitesimal $A$ satisfaga que la familia $\left\{A^{n}\right\}_{n \in \mathbb{N}}$ sea equicontinua. En nuestro 
trabajo el generador es $(\alpha \cdot \nabla+B)$, donde $B$ es una matriz con coeficientes suaves y este generador infinitesimal no satisface esta propiedad. A pesar de ésto se demuestra que las soluciones de la ecuación de Weyl perturbada es un grupo a un parámtero (ver II.8 -b).

De aquí se obtienen los mismos resultados para la ecuación de Dirac, porque ésta se reduce a la ecuación de Weyl perturbada.

Al momento de escribir este trabajo, el teorema B.2 del apéndice $B$ no se encuentra en la literatura $y$, al parecer, es un resultado original. 


\section{Análisis y Síntesis Espectral en subespa- cios $M(n)$-Invariantes de $\mathcal{E}\left(\mathbf{R}^{n}\right)$.}

II.1 Sean $O(n, \mathbb{R})=\left\{A \in G L(n, \mathbb{R}) /{ }^{t} A A=I\right\}=K$ el grupo compacto de rotaciones en $\mathbb{R}^{n}$ y $M(n)$ el grupo de isometrias del espacio euclideano $\mathbb{R}^{n}$. Es muy fácil ver que $g \in G$ si y sólo si hay una rotación $k \in K$ y un vector $x \in \mathbb{R}^{n}$ tales que $g(y)=k \cdot y+x$. La composición de tales isometrías nos permite identificar el grupo de movimiento rígidos $M(n)$ con el producto semidirecto $\mathbb{R}^{n} \times K$, con la operación de grupo $(a, h) \cdot(x, k):=(a+h \cdot x, h k) \in \mathbb{R}^{n} \times K$. Para $(x, k) \in M(n)$ fijo, el operador de translación izquierda se define por

$$
L_{(x, k)}:=\mathcal{E}\left(\mathbb{R}^{n}\right) \rightarrow \mathcal{E}\left(\mathbb{R}^{n}\right),\left(L_{(x, k)} f\right)(y)=f\left(k^{-1}(y-x)\right) .
$$

Por la definición de las seminormas en $\mathcal{E}\left(\mathbb{R}^{n}\right)$ es inmediato que la representación $L_{(\cdot,)}$ de $M(n)$ en el grupo de operadores invertibles acotados en $\mathcal{E}\left(\mathbb{R}^{n}\right)$ es continua. Para $f \in M(n)$ fija, la función $L_{(x, k)} f$ es una función suave en $(x, k) \in M(n)$ y se concluye que la representacion $L_{(\cdot, \cdot)}$ es diferenciable.

II.2 En coordenadas esféricas $r>0, \theta \in S^{n-1}$, el operador de Laplace se puede expresar como

$$
\Delta=\mathcal{L}_{r}+\frac{1}{r^{2}} \mathcal{L}_{s}, \quad \mathcal{L}_{r}=\frac{\partial^{2}}{\partial r^{2}}+\frac{n-1}{r} \frac{\partial}{\partial r}
$$

y $\mathcal{L}_{\mathfrak{s}}$ admite una extensión autoadjunta definida en un subespacio denso de $\mathcal{L}_{2}\left(S^{n-1}\right)$.

Un vector $\mathbf{a} \in \mathbb{C}^{n}$ se dice isotrópico si $\mathbf{a} \cdot \mathbf{a}=0$. El espacio

$E_{m}=\operatorname{gen} \mathbb{C}\left\{\xi \mapsto\langle\mathbf{a}, \xi\rangle^{m}: \mathbf{a}\right.$ es isotrópico, $\left.\xi \in S^{n-1}\right\}, \quad m=0,1,2, \ldots$

satisface (ver [10], [23,a])

(a) $d(m)=\operatorname{dim}_{\mathbf{C}} E_{m}<+\infty$ y $\left.\mathcal{L}_{S}\right|_{E_{m}}=-m(m+n-2) I$,

(b) $L^{2}\left(S^{n-1}\right)=\varlimsup_{m=0}^{+\infty} E_{m}$.

(c) La $K$-representación regular izquierda es irreducible. 
Sea $\langle\mid\rangle_{L^{2}\left(S^{n-1}\right)}$ el producto interno en $L^{2}\left(S^{n-1}\right)$ y tómese una base ortonormal $\left\{S_{m-1}, \ldots, S_{m, d(m)}\right\}$ de $E_{m}$. Por la diferenciabilidad de la $K$-representación y el teorema de Harisch-Chandra sobre la densidad de series de Fourier [War- ], para cada $f \in \mathcal{E}\left(\mathbb{R}^{n}\right)$ la serie

$$
\sum_{m, \nu}^{+\infty} f_{m, \nu}(r) S_{m, \nu}(\Theta), f_{m, \nu}(r)=\left\langle f(r \cdot) \mid S_{m, \nu}\right\rangle_{L^{2}\left(S^{n-1}\right)^{*}}
$$

converge a $f$ en $\mathcal{E}\left(\mathbb{R}^{n}\right)$. Tal descomposición implica que la ecuación $\Delta u=f$ tiene solución en $\mathcal{E}\left(\mathbb{R}^{n}\right)$. Se sigue que $\Delta: \mathcal{E}\left(\mathbb{R}^{n}\right) \rightarrow \mathcal{E}\left(\mathbb{R}^{n}\right)$ es un operador suprayectivo y abierto. En [10] y [23, a] se demuestra que los espacios $\Delta$-característicos $\mathcal{E}_{z}=\left\{f \in \mathcal{E}\left(\mathbb{R}^{n}\right): \Delta f=z^{2} f\right\}$ correspondientes a cada $z \in \mathbb{C}$ son $M(n)$-irreducibles cuando $z \neq 0 \mathrm{y}$ los únicos subespacios cerrados de $\mathcal{E}_{0}$, dependiendo de la dimensión, son el espacio de polinomios armónicos en $n$-variables $x_{1}, \ldots, x_{n}$ de grado $\leq \ell$ o todo $\varepsilon_{0}$.

Denotemos por $\tau_{x} \equiv L_{(x, I)^{*}}$ y $L_{k} \equiv L_{(0, k)}$. Los operadores duales se definen de la manera acostumbrada y empleamos la misma notación para ambos. Consideremos los espacios $K$-simétricos $\mathcal{E}_{K}\left(\mathbb{R}^{n}\right)=\{f \in$ $\left.\mathcal{E}\left(\mathbb{R}^{n}\right): L_{k} f=f, k \in K\right\}$ y $\mathcal{E}_{K}^{\prime}\left(\mathbb{R}^{n}\right)=\left\{T \in \mathcal{E}^{\prime}\left(\mathbb{R}^{n}\right): L_{k} T=T, k \in\right.$ $K\}$.

Sea $d k$ la medida de Haar biinvariante definida sobre $K$ y considere las proyecciones continuas: $P: \mathcal{E}\left(\mathbb{R}^{n}\right) \rightarrow \mathcal{E}_{K}\left(\mathbb{R}^{n}\right),{ }^{t} P: \mathcal{E}^{\prime}\left(\mathbb{R}^{n}\right) \rightarrow \mathcal{E}_{K}^{\prime}\left(\mathbb{R}^{n}\right)$ definidas por

$$
\begin{aligned}
& P f(x)=\int_{K} f\left(k^{-1} x\right) d k ; f \in \mathcal{E}\left(\mathbb{R}^{n}\right), x \in \mathbb{R}^{n} \\
& { }^{t} P T=\int_{K} L_{k} T d k ; T \in \mathcal{E}^{\prime}\left(\mathbb{R}^{n}\right),
\end{aligned}
$$

donde ${ }^{t} P$ es el operador dual de $P$. Se sigue que

$$
\mathcal{E}\left(\mathbb{R}^{n}\right)=\operatorname{ker} P \oplus \mathcal{E}_{K}\left(\mathbb{R}^{n}\right), \quad \mathcal{E}^{\prime}\left(\mathbb{R}^{n}\right)=\operatorname{ker}^{t} P \oplus \mathcal{E}_{K}^{\prime}\left(\mathbb{R}^{n}\right)
$$

Todos los espacios relacionados en las últimas fórmulaș son $\left(\mathcal{E}_{K}\left(\mathbb{R}^{n}\right), *\right)$ módulos. Ya que la esfera $S^{n-1}$ es difeomorfa a $O(n, \mathbb{R}) / O(n-1, \mathbb{R})$ 
y la medida de Lebesgue en $S^{n-1}$ es $K$-invariante, entonces $P f$ es la media esférica de $f$ y puede calcularse como

$$
P f(x)=\int_{S^{n-1}} f(\|x\| \xi) d s_{n-1}(\xi) ; \quad x \in \mathbb{R}^{n},
$$

donde $d s_{n-1}$ es la medida de superficie normalizada en la esfera unitaria $S^{n-1} \subset \mathbb{R}^{n}$. El operador de Laplace satisface

$$
\Delta=\sum_{j=1}^{n} \frac{\partial^{2}}{\partial x_{j}^{2}}, \quad P \Delta=\Delta P \quad \text { y } \quad L_{(x, k)} \Delta=\Delta L_{(x, k)} .
$$

Por el teorema de derivación bajo el signo de integral se verifica rápidamente que para cada $f \in \mathcal{E}\left(\mathbb{R}^{n}\right)$ la función

$$
\left(\mathcal{I}^{-1} f\right)(r)=\int_{S^{n-1}} f(r \xi) d s_{n-1}(\xi), \quad r \in \mathbb{R}
$$

es una función par suave y se cumple

$$
\left\|\mathcal{I}^{-1} f\right\|_{\ell} \leq \int_{S^{n-1}}\|f(r \xi)\|_{\ell} d s_{n-1}(\xi)=\|f\|_{\ell}
$$

Recíprocamente, si $g$ es una función par suave, por un teorema bien conocido de Whitney, hay una función suave $h \in C^{\infty}(\mathbb{R})$ tal que $g(r)=$ $h\left(r^{2}\right)$. Se sigue que la aplicación $u(x)=g(\|x\|), x \in \mathbb{R}^{n}$; es suave $\mathbf{y}$ $K$-simétrica. Puesto que $\left(I^{-1} u\right)=g$, se sigue que el operador

$$
\mathcal{I}^{-1}: \mathcal{E}_{K}\left(\mathbb{R}^{n}\right) \rightarrow \mathcal{E}\left(\mathbb{R} / \mathbf{Z}_{2}\right)
$$

es continuo y sobre. Aplicando el Teorema del operador abierto se concluye que el operador inverso

$$
\mathcal{I}: \mathcal{E}\left(\mathbb{R} / \mathbb{Z}_{2}\right) \underset{\sim}{\rightarrow} \mathcal{E}_{K}(\mathbb{R}), \quad\left(I_{g}\right)(x)=g(\|x\|)
$$

es continuo.

II.3 En el resto de este trabajo $d t$ representará la medida de Lebesgue en el intervalo $[-1,1]$ normalizada de manera tal que

$$
\int_{-1}^{1}\left(1-t^{2}\right)^{\frac{n-3}{2}} d t=1, \quad n \geq 2
$$


tomando en cuenta lo anterior, definamos

$$
\varphi(\dot{r})=\int_{-1}^{1}\left(1-t^{2}\right)^{\frac{n-3}{2}} e^{-i r t} d t, r \in \mathbb{R}
$$

entonces $\varphi^{\prime \prime}(r)+\frac{(n-1)}{r} \varphi^{\prime}(r)+\varphi(r)=0, \varphi(0)=1$.

Esta ecuación tiene una y sólo una solución suave, a saber:

$$
\varphi(r)=\Gamma\left(\frac{n}{2}\right) \sum_{\nu=0}^{+\infty} \frac{(-1)^{\nu} r^{2 \nu}}{\nu ! 4^{\nu} \Gamma\left(\frac{n}{2}+\nu\right)}
$$

Ahora, dados $\lambda \in \mathbb{C}$ y $x \in \mathbb{R}^{n}$, sea

$$
\phi_{\lambda}(x)=\int_{S^{n-1}} e^{-i \lambda(x, \xi)} d s_{n-1}(\xi)
$$

entonces $\phi_{\lambda} \in \mathcal{E}_{K}\left(\mathbb{R}^{n}\right)$ y $\phi_{\lambda}(0)=1$. De esta manera, las relaciones (II.2.6) y (II.2.7) implican que para $z \in \mathbb{C}^{n}$ y $\lambda \in \mathbb{C}$ con $\lambda^{2}=\mathbf{z} \cdot z=$ $z_{1}^{2}+\cdots+z_{n}^{2}$ se cumple

$$
\Delta P\left(e_{ \pm z}\right)=-\lambda^{2} P\left(e_{ \pm z}\right) \text { y } \Delta \phi_{\lambda}=-\lambda^{2} \phi_{\lambda}
$$

Por lo tanto, la $K$-simetría y la existencia y unicidad de soluciones para e.d.o. nos dan

$$
P\left(e_{ \pm \xi}\right)(x)=\phi_{\lambda}(x)=\varphi(\lambda\|x\|)=\Gamma\left(\frac{n}{2}\right) \sum_{\nu=0}^{+\infty} \frac{(-1)^{\nu}(\lambda\|x\|)^{2 \nu}}{\nu ! 4^{\nu} \Gamma\left(\frac{n}{2}+\nu\right)}
$$

Si $\lambda \in \mathbb{C}$ es fijo y $j=0,1,2, \ldots$, definimos

$$
\phi_{\lambda, j}(x)= \begin{cases}\left.\frac{\partial^{j} \phi_{z}(x)}{\partial z^{j}}\right|_{z=\lambda}, & \lambda \neq 0 \\ \|x\|^{2 j}, & \lambda=0\end{cases}
$$

y

$$
\mathcal{E}_{\lambda}^{(j)}=\left\{f \in \mathcal{E}\left(\mathbb{R}^{n}\right) \mid\left(\Delta+\lambda^{2}\right)^{j+1} f=0\right\} .
$$

Para $\lambda \neq 0, \phi_{\lambda, j}$ satisface

$$
\Delta \phi_{\lambda, j}=-\lambda^{2} \phi_{\lambda, j}-2 \lambda j \phi_{\lambda, j-1}-j(j-1) \phi_{\lambda, j-2} ; j=0,1,2, \ldots,
$$


donde, por definición, $\phi_{\lambda_{1}-1}=\phi_{\lambda_{1}-2}=0$. En consecuencia

$$
\left(\Delta+\lambda^{2}\right) \phi_{\lambda, 0}=0 \text { y }\left(\Delta+\lambda^{2}\right)^{j} \phi_{\lambda, j}=(-2 \lambda)^{j} ! \phi_{\lambda, 0}, j \geq 1 .
$$

Se sigue que $\phi_{\lambda, 0}, \ldots, \phi_{\lambda, j} \in \mathcal{E}_{\lambda}^{(j)}$. Puesto que los operadores $\tau_{x}, L_{k}$ satisfacen $L_{k-1} \tau_{x} L_{k}=\tau_{k^{-1} x}$, entonces para cada $T \in \mathcal{E}^{\prime}\left(\mathbb{R}^{n}\right)$ y $\phi \in$ $\mathcal{E}_{K}\left(\mathbb{R}^{n}\right)$ tendremos $L_{k}(T * \phi)=\left(L_{k-1} T\right) * \phi$ y concluímos que el espacio $X_{j}=\overline{\mathcal{E}^{\prime}\left(\mathbb{R}^{n}\right) *\left\{\phi_{\lambda, 0}, \ldots, \phi_{\lambda, j}\right\}}$ es $M(n)$-invariante. Se tiene el siguiente

II.4 Teorema. Para todo $0 \neq \lambda \in \mathbb{C}$ y todo $m=0,1,2, \ldots$ se cumple

$$
X_{m}=\mathcal{E}_{\lambda}^{(m)} \text {. }
$$

Demostración. Aplicamos el principio de inducción.

En el caso $m=0, X_{0}$ es un subespacio $M(n)$-invariante del espacio irreducible $\mathcal{E}_{\lambda}$. Ya que $X_{0}$ es cerrado y no es nulo, entonces $X_{0}=\mathcal{E}_{\lambda}$.

Supongamos ahora que la afirmación es cierta para $0 \leq j<m$. Sabemos que $\left(\Delta+\lambda^{2}\right)$ es un operador abierto suprayectivo, entonces $(\Delta+$ $\left.\lambda^{2}\right)^{j+1}$ induce un isomorfismo topológico

$$
\mathcal{E}_{\lambda}^{(j+1)} / \mathcal{E}_{\lambda}^{(j)} \stackrel{\left(\Delta+\lambda^{2}\right)^{j+1}}{\approx} \mathcal{E}_{\lambda}^{(0)}
$$

Mas aún, en clases de equivalencia, $\left(\Delta+\lambda^{2}\right)^{j+1}$ es un operador de enlace (intertwining operator) entre las respectivas representaciones izquierdas de $M(n)$. Por hipótesis de inducción $\mathcal{E}_{\lambda}^{(j)}=X_{j} \subsetneq X_{j+1} \subset \mathcal{E}_{\lambda}^{(j+1)}$, por lo tanto $X_{j+1} / \mathcal{E}_{\lambda}^{(j)}$ es un subespacio cerrado $M(n)$-invariante de $\mathcal{E}_{\lambda}^{(j+1)} / \mathcal{E}_{\lambda}^{(j)}$ que no es nulo. Por (II.4.1) el último cociente es irreducible y de aquí $X_{j+1}=\mathcal{E}_{\lambda}^{(j+1)}$.

El teorema precedente y (II.3.8) nos dan

II.5 Corolario. Con la misma notación $\mathcal{E}_{\lambda}^{(m)}=\overline{\mathcal{E}^{\prime}\left(\mathbb{R}^{n}\right) *\left\{\phi_{\lambda, m}\right\}}$. 
II.6 Consideremos el espacio de funciones pares suaves $\mathcal{E}\left(\mathbb{R} / \mathbb{Z}_{2}\right) \subset \mathcal{E}(\mathbb{R})$ y los siguientes operadores continuos:

$$
\begin{gathered}
\mathcal{E}\left(\mathbb{R} / \mathbb{Z}_{2}\right) \stackrel{\mathcal{I}}{\sim} \mathcal{E}_{K}\left(\mathbb{R}^{n}\right), \quad(\mathcal{I} f)(x)=f(|x|) \\
B: \mathcal{E}\left(\mathbb{R} / \mathbb{Z}_{2}\right) \rightarrow \mathcal{E}\left(\mathbb{R} / \mathbb{Z}_{2}\right), \quad B f(r)=\int_{-1}^{1}\left(1-t^{2}\right)^{\frac{n-3}{2}} f(r t) d t
\end{gathered}
$$

Observemos que para $f \in \mathcal{E}(\mathbb{R})$ se tiene:

$$
\frac{d^{2} B f}{d r^{2}}+\frac{(n-1)}{r} \frac{d B f}{d r}=\mathcal{L}_{r} B f=B f^{\prime \prime} .
$$

$\mathrm{Si}$

$$
u(t, r)=\int_{-1}^{1}\left(1-s^{2}\right)^{\frac{n-3}{2}} f(t+r s) d s
$$

entonces $u(t, 0)=f(t) \mathrm{y}$

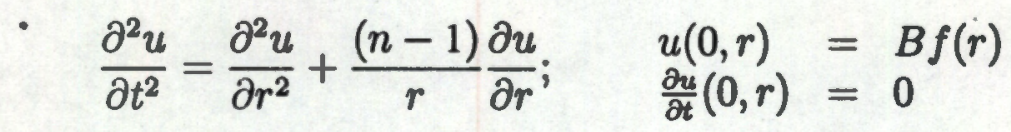

Por existencia y unicidad para ecuaciones hiperbólicas, el problema (II.6.4) tiene solución única y, por lo tanto, $B$ es un operador invertible con continuidad. Como se sabe la solución de (II.6.4) se obtiene por el método descendente en la dimensión y aquí se omite. En particular para $n=3$,

$$
B^{-1} g=\frac{\partial}{\partial r}(r g), g \in \mathcal{E}\left(\mathbb{R} / \mathbb{Z}_{2}\right) .
$$

En lugar de obtener una expresión para $B^{-1}$ tenemos

II.7 Lema. En el siguiente diagrama conmutativo

$$
\begin{array}{lcc}
\mathcal{E}\left(\mathbb{R} / \mathbb{Z}_{2}\right) & \stackrel{B}{\rightarrow} & \mathcal{E}\left(\mathbb{R} / \mathbb{Z}_{2}\right) \\
\mathcal{I} B \searrow & \downarrow \mathcal{I} \\
& & \mathcal{E}_{K}\left(\mathbb{R}^{n}\right)
\end{array}
$$


el operador dual de $\mathcal{I} B,(\mathcal{I} B)^{\prime}$ es un isomorfismo topológico algebraico entre $\left(\mathcal{E}_{K}^{\prime}\left(\mathbb{R}^{n}\right), *_{n}\right)$ y $\left(\mathcal{E}^{\prime}\left(\mathbb{R} / \mathbb{Z}_{2}\right), *_{1}\right)$, donde $*_{m}$ denota la convolución $m$-dimensional.

Demostración. De los comentarios previos se deduce que $I B$ es un isomorfismo topológico y de aquí $(I B)^{\prime}$ lo es también. Para demostrar que $(\mathcal{I} B)^{\prime}$ es un isomorfismo de anillos fijamos $T \in \mathcal{E}_{K}^{\prime}\left(\mathbb{R}^{n}\right)$ y calculamos la 1-transformada de Fourier de $(\mathcal{I} B)^{\prime} T \in \mathcal{E}^{\prime}\left(\mathbb{R} / \mathbf{Z}_{2}\right)$ : En virtud de (II.3.5) y (II.3.1),

$$
\left[(\mathcal{I} B)^{\prime} T\right]^{\wedge}(\lambda)=\left[(\mathcal{I} B)^{\prime} T\right]\left(e_{-\lambda}\right)=T\left(\mathcal{I} B e_{-\lambda}\right)=T\left(\phi_{\lambda}\right), \quad \lambda \in \mathbb{C}
$$

Así, dados $T_{1}, T_{2} \in \mathcal{E}_{K}^{\prime}\left(\mathbb{R}^{n}\right)$, la propiedad $P\left(T_{1} *_{n} T_{2}\right)=T_{1} *_{n} T_{2}$ nos dá

$$
\begin{array}{ll}
\begin{array}{ll}
{\left[(\mathcal{I} B)^{\prime}\left(T_{1} *_{n} T_{2}\right)\right]^{\wedge}(\lambda)} & =\left(T_{1} *_{n} T_{2}\right)\left(\phi_{\lambda}\right)=T_{1}\left(\phi_{\lambda}\right) T_{2}\left(\phi_{\lambda}\right) \\
& =\left[(I B)^{\prime} T_{1}\right]^{\wedge}(\lambda)\left[(I B)^{\prime} T_{2}\right]^{\wedge}(\lambda)
\end{array} \\
\text { por lo tanto }
\end{array}
$$

$$
(\mathcal{I} B)^{\prime}\left(T_{1} *_{n} T_{2}\right) \quad=\left[(\mathcal{I} B)^{\prime} T_{1}\right] *_{1}\left[(\mathcal{I} B)^{\prime} T_{2}\right] .
$$

Hasta aquí la prueba está completa, sin embargo, es conveniente hallar la relación entre las transformadas de Fourier de $(\mathcal{I} B)^{\prime} T \in \mathcal{E}^{\prime}\left(\mathbb{R} / \mathbb{Z}_{2}\right)$ y $T \in \mathcal{E}_{K}^{\prime}\left(\mathbb{R}^{n}\right)$ respectivamente:

Si $\lambda \in \mathbb{C}$ y $z \in \mathbb{C}^{n}$ son tales que $\lambda^{2}=z \cdot z=z_{1}^{2}+\cdots+z_{n}^{2}$, entonces $\phi_{\lambda}=P\left(e_{-z}\right)$ y

$$
\begin{aligned}
\hat{T}(\mathfrak{\jmath})=T\left(e_{-\jmath}\right) & =P T\left(e_{-\jmath}\right)=T\left(P e_{-\jmath}\right)=T\left(\phi_{\lambda}\right) \\
& =\left[(\mathcal{I} B)^{\prime} T\right]^{\wedge}(\lambda)
\end{aligned}
$$

Ahora, $\left[(\mathcal{I} B)^{\prime} T\right]^{\wedge}$ es una función par entera en $\mathbb{C}$ por lo tanto

$$
\left[(\mathcal{I} B)^{\prime} T\right]^{\wedge}(\lambda)=\sum_{\nu=0}^{+\infty} a_{\nu} \lambda^{2 \nu}
$$


De esta manera (II.7.2) y la unicidad de series de potencias nos permiten concluir

$$
\hat{T}(z)=\sum_{\nu=0}^{+\infty} a_{\nu}(z \cdot z)^{\nu}
$$

II.SAS. Sea $0 \neq W \subset \mathcal{E}(\mathbb{R})$ un subespacio lineal cerrado invariante bajo translaciones y escribamos

$$
\begin{aligned}
\mathfrak{a} & =\left\{T \in \mathcal{E}^{\prime}(\mathbb{R}) \mid T * \check{f}=0, f \in W\right\} \\
\sigma(\mathfrak{a}) & =\{\lambda \in \mathbb{C} \mid \hat{T}(\lambda)=0, \quad T \in \mathfrak{a}\} .
\end{aligned}
$$

A cada $\lambda \in \sigma(\mathfrak{a})$ asignamos

$$
m(\lambda)=\max \left\{m \in \mathbf{Z} \mid \hat{T}^{(j)}(\lambda)=0,0 \leq j \leq m, T \in \mathfrak{a}\right\} .
$$

El Teorema de Análisis y Síntesis espectral de L. Schwartz, [20, a], asegura:

(a) El espectro de a, $\sigma(\mathfrak{a})$, no es vacío.

(b) $\mathrm{Si}$ " $\Sigma$ " denota la suma directa algebraica de espacios lineales y $W \neq \mathcal{E}(\mathbb{R})$ entonces

$$
W=\overline{\sum_{\lambda \in \sigma(\mathfrak{a})} \mathbf{C}\left\langle M^{j} e_{-\lambda} \mid 0 \leq j \leq m(\lambda)\right\rangle}
$$

donde

$$
M^{j} e_{-\lambda}(x)=x^{j} e^{-i \lambda x}, x \in \mathbb{R} .
$$

Por brevedad, al referirnos a este teorema sólo escribiremos SAS y el conjunto $\{\sigma(\mathfrak{a}), m(\lambda)\}:=\{(\lambda, m(\lambda)) \mid \lambda \in \sigma(\mathfrak{a})\}$ será llamado la "variedad definiente de $a$ ".

Dado un subespacio lineal cerrado $M(n)$-invariante $0 \neq V \neq \mathcal{E}\left(\mathbb{R}^{n}\right)$ escribimos $V_{0}=V \cap \mathcal{E}_{K}\left(\mathbb{R}^{n}\right)$ entonces

$$
V_{0}=P V \text { y } V=(V \cap \operatorname{ker} P) \oplus V_{0}^{\prime}
$$


La invariancia translacional de $V$ nos da

$$
V=\mathcal{E}^{\prime}\left(\mathbb{R}^{n}\right) * V \text { y } V_{0}=\mathcal{E}_{K}^{\prime}\left(\mathbb{R}^{n}\right) * V_{0}
$$

Definamos

$$
\begin{aligned}
& V^{\perp}=\left\{T \in \mathcal{E}^{\prime}\left(\mathbb{R}^{n}\right) \mid T * \check{f}=0, f \in V\right\} \\
& V^{\perp}=\left\{T \in \mathcal{E}_{K}^{\prime}\left(\mathbb{R}^{n}\right) \mid T * \check{f}=0, f \in V_{0}\right\} \\
& V^{\perp \perp}=\left\{f \in \mathcal{E}\left(\mathbb{R}^{n}\right) \mid T * \check{f}=0, f \in V^{\perp}\right\} \\
& V^{\perp \perp}=\left\{f \in \mathcal{E}_{K}\left(\mathbb{R}^{n}\right) \mid T * \check{f}=0, f \in V_{0}^{\perp}\right\}
\end{aligned}
$$

Los espacios $\mathcal{E}\left(\mathbb{R}^{n}\right), \mathcal{E}^{\prime}\left(\mathbb{R}^{n}\right)$ son reflexivos luego, el teorema de HahnBanach implica $V^{\perp \perp}=V$ y $V_{0}^{\perp \perp}=V_{0}$

Ahora consideramos

$$
\begin{aligned}
& W_{0}=(\mathcal{I} B)^{-1} V_{0} \subset \mathcal{E}\left(\mathbb{R} / \mathbf{Z}_{2}\right) ; \quad W=\overline{\mathcal{E}^{\prime}(\mathbb{R}) * W_{0}}, \\
& \mathfrak{a}_{0}=\left\{T \in \mathcal{E}^{\prime}\left(\mathbb{R} / \mathbf{Z}_{2}\right) \mid T * \tilde{f}=0, f \in W_{0}\right\}
\end{aligned}
$$

Las siguientes proyecciones continuas

$$
\begin{array}{ll}
\mathcal{E}(\mathbb{R}) \stackrel{P_{0}}{\longrightarrow} \mathcal{E}\left(\mathbb{R} / \mathbb{Z}_{2}\right) ; & P_{0}(f)=\frac{f+\check{f}}{2} \\
\mathcal{E}^{\prime}(\mathbb{R}) \stackrel{P_{0}^{\prime}}{\longrightarrow} \mathcal{E}\left(\mathbb{R} / \mathbb{Z}_{2}\right) ; & P_{0}(T)=\frac{T+\check{T}}{2}
\end{array}
$$

satisfacen: $W$ es $\mathbb{Z}_{2}$-invariante $\mathbf{y}$

$$
\begin{aligned}
& W_{0}=P_{0}(W) ; \quad W=\left(W \cap \operatorname{ker} P_{0}\right) \oplus W_{0} \\
& \mathfrak{a}_{0}=P_{0}^{\prime}(\mathfrak{a}) ; \quad \mathfrak{a}=\left(\mathfrak{a} \cap \operatorname{ker} P_{0}^{\prime}\right) \oplus \mathfrak{a}_{0} .
\end{aligned}
$$

Hagamos el análisis espectral de $V$.

II.8 Teorema. Con la notación previa tenemos que $\sigma(\mathfrak{a})$ no es vacío y

(1) Para cada $0 \neq \lambda \in \sigma(a), \mathcal{E}_{\lambda}^{m(\lambda)} \subset V$.

(2) Si $\lambda=0$ pertenece a $\sigma(a)$, entonces $m(0)$ es un número par y

$$
V \cap \mathcal{E}_{0}^{\left(\frac{m(0)}{2}\right)} \neq \phi
$$


Demostración. Sea $f \in V, f \neq 0$. Hay un punto $x_{0} \in \mathbb{R}^{n}$ tal que $f\left(x_{0}\right) \neq 0$ y de aqui $P\left(\tau_{-x_{0}} f\right)(0)=f\left(x_{0}\right) \neq 0$. Se sigue que $V_{0}=P(V) \neq 0$. Yá que (II.7.3) nos dá $W \neq 0$, la parte (a) de SAS implica $\sigma(\mathfrak{a}) \neq \phi$. Para cualquier $\lambda \in \sigma(\mathfrak{a})$ la fórmula (S.2) asegura que $M^{j} e_{-\lambda} \in W$ para $0 \leq j \leq m(\lambda)$, por lo tanto $P_{0}\left(M^{j} e_{-\lambda}\right) \in W_{0}$ para $0 \leq j \leq m(\lambda)$. Observando que $B P_{0}=B$ obtenemos

$$
\left.\frac{\partial^{j} \phi_{z}}{\partial z^{j}}\right|_{z=\lambda}=(-i)^{j} \mathcal{I} B\left(P_{0}\left(M^{j} e_{-\lambda}\right)\right) \in V_{0}, \quad 0 \leq j \leq m(\lambda)
$$

(1) Cuando $\lambda \neq 0$, la definición de $\phi_{\lambda, j}$ en (II.3.6), el teorema II.4 y la invariancia bajo traslaciones de $V$ nos llevan a

$$
\mathcal{E}_{\lambda}^{(m(\lambda))}=\overline{\mathcal{E}^{\prime}\left(\mathbb{R}^{n}\right) *\left\{\phi_{\lambda, 0}, \ldots, \phi_{\lambda, m(\lambda)}\right\}} \subseteq \overline{\mathcal{E}^{\prime}\left(\mathbb{R}^{n}\right) * V_{0}} \subset V
$$

Para mostrar (2) obsérvese primero que $B$ se anula en funciones impares. En segundo lugar, la relación (S.2) muestra que $W$ es el único subespacio que contiene todas las funciones $M^{j} e_{-\lambda}$. Por definición de $W$, éste es el mínimo subespacio cerrado de $\mathcal{E}(\mathbb{R})$ que es invariante bajo translaciones y contiene a $W_{0}$; por consiguiente $m(0)=2 \ell$ para algún entero $\ell \geq 0$ y $M^{2 j} \in V_{0}, 0 \leq j \leq \ell$. Se concluye que

$$
\|\|^{2 j}=\mathcal{I} B\left(M^{2 j}\right) \in V_{0} \subset V, \quad j=0,1, \ldots, \ell,
$$

además $\Delta^{\ell+1}\|\|^{2 j}=0, j=0,1, \ldots, \ell$.

Para obtener la síntesis espectral de $V$ necesitamos algunos resultados preliminares:

II.9 Teorema. Para cada $z \notin \sigma(a)$ el operador diferencial $\left(\Delta+z^{2}\right): V \rightarrow$ $V$ tiene un inverso continuo. Si $R_{z}=\left.\left(\Delta+z^{2}\right)\right|_{V} ^{-1}$, entonces existen $T, H \in \mathcal{E}_{K}^{\prime}\left(\mathbb{R}^{n}\right)$ tales que $T \in V^{\perp}, \hat{T}\left(\phi_{z}\right) \neq 0$ y

$$
R_{z} f=\frac{H * f}{T\left(\phi_{z}\right)}, \quad f \in V
$$


Demostración. Por la definición de $\sigma(a)$ se tiene la existencia de algún $T_{0} \in a_{0}$ tal que $\hat{T}_{0}(z) \neq 0$. $\hat{T}_{0}$ es una función par entera y por lo tanto la aplicación $\xi \rightarrow\left(\left(\hat{T}_{0}(\xi)-\hat{T}_{0}(z)\right) /\left(\xi^{2}-z^{2}\right)\right)$ es entera y par así que, en virtud del Teorema de Ehrenpreis-Malgrange, hay un $H_{0} \in \mathcal{E}^{\prime}\left(\mathbb{R} / \mathbb{Z}_{2}\right)$ tal que

$$
\hat{T}_{0}(\xi)-\hat{T}_{0}(z)=\left(\xi^{2}-z^{2}\right) \hat{H}_{0}(\xi), \quad \xi \in \mathbb{C}
$$

Escribiendo $T=(\mathcal{I} B)^{\prime-1} T_{0}$ y $H=(\mathcal{I} B)^{\prime-1} H_{0}$, entonces el lema II.7 muestra que $T, H \in \mathcal{E}_{K}^{\prime}\left(\mathbb{R}^{n}\right)$ y $T \in V_{0}^{ \pm} \subset V^{\perp}$. Además $T$ y $H$ satisfacen

$$
\hat{T}(\mathfrak{z})=T\left(\phi_{\xi}\right)=\hat{T}_{0}(\xi) ; \hat{H}(z)=H\left(\phi_{\xi}\right)=\hat{H}(\xi) ; \operatorname{para} \xi^{2}=z \cdot z
$$

De esta manera la relación (II.9.2) se reduce a

$$
\hat{T}(z)-T\left(\phi_{z}\right)=\left(z \cdot z-z^{2}\right) \hat{H}(z), \quad z \in \mathbb{C}^{n}
$$

Por lo tanto en el sentido distribucional tendremos

$$
T-T_{0}\left(\phi_{z}\right) \delta=-\left(\Delta+z^{2}\right) H .
$$

Si aplicamos ambos lados de esta igualdad a cualquier $f \in V$ y recordando que $T * \breve{f}=0$ obtendremos

$$
T_{0}\left(\phi_{z}\right) \check{f}=T_{0}\left(\phi_{z}\right) \delta * \check{f}=\left(\Delta+z^{2}\right) H * \check{f}
$$

i.e.,

$$
f=\left(\Delta+z^{2}\right) \frac{\check{H} * f}{T\left(\phi_{z}\right)}=\left(\Delta+z^{2}\right) \frac{H * f}{T\left(\phi_{z}\right)} ; f \in V
$$

Ya que $H * f \in V$, nuestra afirmación se sigue de la última ecuación.

II.10 Observación. La $K$-simetría de $H$ y la propiedad $P(H * g)=H * P(g)$ para $g \in \mathcal{E}\left(\mathbb{R}^{n}\right)$ establece la propiedad conmutativa $R_{z} P=P R_{z}$. Por lo tanto la unicidad de soluciones implica que $\left.\left(\Delta+z^{2}\right)\right|_{P(V)}$ tiene un inverso continuo en $P(V)$.

De aquí en adelante $\lambda$ y $m$ denotarán $\lambda \in \sigma(a)$ y $m=m(\lambda)$ respectivamente. 
II.11 Teorema. Sean $\lambda \in \sigma(\mathfrak{a})$ y $X_{\lambda}=\left(\Delta+\lambda^{2}\right)^{m+1} V$. Si $X_{\lambda} \neq 0$, entonces el operador diferencial $\left(\Delta+\lambda^{2}\right): X_{\lambda} \rightarrow X_{\lambda}$ tiene un inverso continuo.

Demostración. Distinguimos dos casos:

(a) $\lambda \neq 0$. Por la definición de $m=m(\lambda)$ podemos encontrar $T \in$ a tal que $\hat{T}^{(j)}(\lambda)=0, \quad j=0,1, \ldots, m$ y $\hat{T}^{(m+1)}(\lambda) \neq 0$, i.e., $M^{m+1} e_{-\lambda} \notin W$. Ya que $\left(M^{m+1} e_{\lambda}\right)^{\vee}=2\left(P_{0}\left(M^{m+1} e_{-\lambda}\right)-\right.$ $\left.M^{m+1} e_{-\lambda}\right)$, entonces $-\lambda \in \sigma(\mathfrak{a})$ y $m(-\lambda)=m(\lambda)$. Por lo tanto $T_{0}=P_{0}(T)$ satisface $\hat{T}_{0}^{(j)}(\lambda)=0,0 \leq j \leq m$ y $\hat{T}_{0}^{(m+1)}(\lambda) \neq 0$. Por consiguiente hay un $S_{0} \in \mathcal{E}^{\prime}\left(\mathbb{R} / \mathbf{Z}_{2}\right)$ tal que

$$
\hat{T}_{0}(\xi)=\left(\xi^{2}-\lambda^{2}\right)^{m+1} \hat{S}_{0}(\xi), \hat{S}_{0}(\lambda) \neq 0
$$

y $\hat{S}_{0}(\xi)=\hat{S}_{0}(\lambda)+\left(\xi^{2}-\lambda^{2}\right) \hat{H}_{0}(\xi)$ por lo tanto

$$
\hat{T}_{0}(\xi)=\left(\xi^{2}-\lambda^{2}\right)^{m+1}\left[\hat{S}_{0}(\lambda)+\left(\xi^{2}-\lambda^{2}\right) \hat{H}_{0}(\xi)\right] .
$$

Si hacemos $T_{0}=(\mathcal{I} B)^{\prime} T_{1}$ y $H_{0}=(\mathcal{I} B)^{\prime} H$ entonces (II.11.2) puede reformularse como

$$
T_{1}=-\left(\Delta+\lambda^{2}\right)^{m+1}\left[\hat{S}_{0}(\lambda) \delta-\left(\Delta+\lambda^{2}\right) H\right] \in V^{\perp} .
$$

De aquí, para cada $f \in V$ se tendrá $T_{1} * \check{f}=0$ y el segundo miembro nos dará

$$
\left(\Delta+\lambda^{2}\right)^{m+1} f=\left(\Delta+\lambda^{2}\right) \frac{H *\left[\left(\Delta+\lambda^{2}\right)^{m+1} f\right]}{\hat{S}_{0}(\lambda)}, \quad f \in V
$$

por lo tanto

$$
g=\left(\Delta+\lambda^{2}\right) \frac{H * g}{\hat{S}_{0}(\lambda)}, \quad g \in X_{\lambda}
$$

(b) Cuando $\lambda=0$ sabemos que $m=m(0)=2 \ell$ para algún entero $\ell \geq 0$ y (II.11.1) adquiere la forma

$$
\hat{T}_{0}(\xi)=\xi^{2 \ell} \hat{S}_{1}(\xi)
$$

donde $S_{1} \in \mathcal{E}\left(\mathbb{R} / \mathbb{Z}_{2}\right), \hat{S}_{1}(0) \neq 0$. El razonamiento empleado en (a) nos conduce a la existencia de algún $G \in \mathcal{E}_{K}^{\prime}\left(\mathbb{R}^{n}\right)$ tal que

$$
g=\Delta \frac{G * g}{\hat{S}_{1}(0)}, \quad g \in X_{0}
$$


Como consecuencia inmediata de los resultados precedentes obtenemos

II.12 Corolario. Con las.mismas hipótesis para $X_{\lambda}$, sean $L_{\lambda}:=\left.\left(\Delta+\lambda^{2}\right)\right|_{X_{\lambda}} ^{m+1}$ y $\Gamma_{\lambda}:=L_{\lambda}^{-1}$ entonces:

(1) $\left.L_{\lambda} \Gamma_{\lambda}\left(\Delta+\lambda^{2}\right)\right|_{V} ^{m+1}=L_{\lambda}$

(2) El operador lineal $q_{\lambda}: V \rightarrow V$ definido por

$$
q_{\lambda}=I-\left.\Gamma_{\lambda}\left(\Delta+\lambda^{2}\right)\right|_{V} ^{m+1}
$$

es una proyección continua cuyo núcleo es $X_{\lambda}$.

(3) El rango de $q_{\lambda}, \Re\left(q_{\lambda}\right)$, satisface

$$
\Re\left(q_{\lambda}\right)=V \cap \mathcal{E}_{\lambda}^{m}= \begin{cases}\mathcal{E}_{\lambda}^{(m)}, & \lambda \neq 0 \\ V \cap \mathcal{E}_{0}^{\left(\frac{m(0)}{2}\right)}, & \lambda=0\end{cases}
$$

En particular $V \cdot=\Re\left(q_{\lambda}\right) \oplus X_{\lambda}$.

(4) Para cualesquiera $k \in K$ y $S \in \mathcal{E}^{\prime}\left(\mathbb{R}^{n}\right), S * q_{\lambda}=q_{\lambda} * S$ y $L_{k} q_{\lambda}=$ $q_{\lambda} L_{k}$. En particular $P q_{\lambda}=q_{\lambda} P$.

(5) $P\left(\Re\left(q_{\lambda}\right)\right)=\mathbb{C}\left\langle\phi_{\lambda, 0}, \ldots, \phi_{\lambda, m}\right\rangle$.

II.13 Lema. Si $\mathcal{N}=\underset{\sigma(\mathfrak{a})}{\bigcap_{1}} \operatorname{ker} q_{\lambda}$, entonces $\mathcal{N}=0$.

Demostración. Por (4) del corolario precedente $\mathcal{N}$ es un subespacio lineal cerrado $M(n)$-invariante de $\mathcal{E}\left(\mathbb{R}^{n}\right)$. Si $\mathcal{N} \neq 0$, el teorema II.8 aplicardo a $\mathcal{N}$ nos da la existencia de algún $\alpha \in \mathbb{C}$ tal que $\phi_{\alpha} \in \mathcal{N} \subset V_{0}$. Para $m=m(\alpha)$ se cumple

$$
0=q_{\alpha}\left(\phi_{\alpha}\right)=\phi_{\alpha}-\left.\Gamma_{\alpha}\left(\Delta+\alpha^{2}\right)\right|_{V} ^{m+1} \phi_{\alpha}=\phi_{\alpha},
$$

que es una contradicción.

Como se vió en II.11, $\lambda \in \sigma(\mathfrak{a}) \Leftrightarrow-\lambda \in \sigma(\mathfrak{a})$ y $m(\lambda)=m(-\lambda)$ por consiguiente $q_{\lambda}=q_{-\lambda}$. Si escribimos

$$
\sigma(a)=\{\lambda \in \sigma(a) \mid \lambda=0 \quad 0 \leq \text { argumento }(\dot{\lambda})<\pi\}
$$

entonces la sintesis espectral de $V$ se obtiene por el siguiente 
II.14 Teorema. $V$ está unívocamente determinado por la variedad definiente $\{\underline{\sigma(\mathfrak{a})}, m(\lambda)\}$ y sus proyecciones $q_{\lambda}, \lambda \in \sigma(\mathfrak{a})$.

Además
(a) $W_{0}=\mathbb{C}\left\langle M^{2 j} \mid j=0, \ldots, \frac{m(0)}{2}\right\rangle \oplus \overline{\sum_{0 \neq \lambda \in \sigma(\mathfrak{a})} \mathbb{C}\left(P_{0}\left(M^{j} e_{-\lambda}\right)|0 \leq j \leq m(\lambda)\rangle\right.}$
(b) $V_{0}=\mathbb{C}\left\langle\|\|^{2 j} \mid j=0, \ldots, \frac{m(0)}{2}\right\rangle \oplus \overline{\sum_{0 \neq \lambda \in \sigma(\mathfrak{a})} \mathbb{C}\left\langle\phi_{-\lambda, j} \mid 0 \leq j \leq m(\lambda)\right\rangle}$

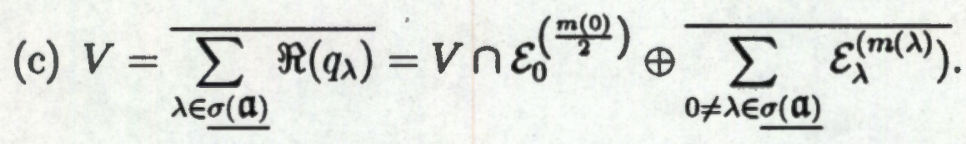

Demostración. Ante todo observemos primero que las fórmulas dadas en (II.2.3) muestran que el único espacio cerrado $M(n)$-invariante que contiene a. $\mathcal{E}_{K}\left(\mathbb{R}^{n}\right)$ es $\mathcal{E}\left(\mathbb{R}^{n}\right)$ y de aquí se sigue que $V_{\theta} \neq \mathcal{E}_{K}\left(\mathbb{R}^{n}\right)$.

(a) Ya que $W_{0}=(\mathcal{I B})^{-1} V_{0}$ y $0 \neq V \neq \mathcal{E}\left(\mathbb{R}^{n}\right)$, entonces $0 \neq V_{0} \neq$ $\mathcal{E}_{K}\left(\mathbb{R}^{n}\right), 0 \neq W_{0} \neq \mathcal{E}\left(\mathbb{R} / \mathbb{Z}_{2}\right)$ y $0 \neq W \neq \mathcal{E}(\mathbb{R})$. La igualdad en (a) es consecuencia inmediata de SAS y la identidad $W_{0}=P_{0}(W)$.

La parte (b) se sigue de (a) y las igualdades

$$
\phi_{\lambda, j}=\mathcal{I B}\left(P_{0}\left(M^{j} \epsilon_{-\lambda}\right)\right) ; \quad \phi_{0, j}=\mathcal{I B}\left(M^{2 j}\right), \quad \text { y } V_{0}=(\mathcal{I B}) W_{0} .
$$

Para obtener (c), sea $U=\overline{\sum_{\boldsymbol{c}(\mathfrak{a})} \Re\left(q_{\lambda}\right)}$ y supongamos $U \neq V$.

Esta condición es equivalente a la existencia de un par $S \in U^{\perp}$ y $f \in V$ tales que $S * \check{f} \neq 0$. Para cada $\lambda \in \sigma\left(\mathfrak{a}^{\perp}\right)$ se tiene que $q_{\lambda}(f) \in U$ y de aquí

$$
\left[q_{\lambda}(\check{S} * f)\right]^{\vee}=\left[\check{S} * q_{\lambda}(f)\right]^{\vee}=S *\left[q_{\lambda} f\right]^{\vee}=0
$$

i.e., $\check{S} * f \in \operatorname{ker} q_{\lambda}$, todo $\lambda \in \sigma(\mathfrak{a})$. Por el lema, $h=\check{S} * f=0$ y obtenemos $S * \check{f}=\check{h}=0$. Esta contradicción asegura que $U=V$. Ahora (3) del corolario II.12 nos da la segunda igualdad y la demostración está completa. 
II.15 Calculemos la proyección $q_{\lambda}$. En el caso $\lambda \neq 0$, escoja $T_{0} \in a_{0}$. Por definición de $m(\lambda), \hat{T}_{0}$ tiene un cero en $\lambda$ de orden $\ell \geq m(\lambda)+1$. Aplicando el Teorema de Ehrenpreis-Malgrange vemos que

$$
\hat{H}_{0}(z)=\frac{\hat{T}_{0}(z)}{\left(z^{2}-\lambda^{2}\right)^{\ell}}, \quad \hat{H}_{j}(z)=\left(z^{2}-\lambda^{2}\right)^{j} \hat{H}_{0}(z), j=1, \ldots, m
$$

satisface $H_{j} \in \mathcal{E}^{\prime}\left(\mathbb{R} / \mathbb{Z}_{2}\right)$ y

$$
\hat{H}_{0}(\lambda) \neq 0 ; \quad \hat{H}_{j}^{(j)}(\lambda)=j !(2 \lambda)^{j} \hat{H}_{0}(\lambda) \neq 0 ; \quad j=1, \ldots, m .
$$

Definiendo $L_{j}=\left[(\mathcal{I B})^{-1}\right]^{\prime} H_{j} \in \mathcal{E}_{K}^{\prime}\left(\mathbb{R}^{n}\right)$, entonces $L_{j}=(-1)^{j}(\Delta+$ $\left.\lambda^{2}\right)^{j} L_{0}$ y para cada $z \in \mathbb{C}, z \neq 0, L_{j}\left(\phi_{z, \nu}\right)=\hat{H}^{\nu}(z)$. En particular para cada $\gamma \in \sigma(\mathfrak{a}), \quad \gamma \neq \lambda$,

$$
L_{j}\left(\phi_{\gamma, \nu}\right)=\hat{H}^{(\nu)}(\gamma)=0, \quad \nu=0, \ldots, m(\gamma)
$$

Por lo tanto $L_{j} \in X_{\lambda}^{\frac{1}{1}}$ y

$$
L_{j}\left(q_{\lambda} g\right)=L_{j}(g)=L_{j}(P g), \quad g \in V .
$$

Para $f \in V$ y $x \in \mathbb{R}^{n}$ fijas hay coeficientes $a_{\lambda, j}(f, x), 0 \leq j \leq m$ tales que

$$
P\left(q_{\lambda}\left(\tau_{-x} f\right)\right)=q_{\lambda} P\left(\tau_{-x} f\right)=\sum_{\nu=0}^{m} a_{\lambda, \nu}(f, x) \phi_{\lambda, \nu}
$$

De esta manera las fórmulas (II.15.3) y (II.15.4) nos dan

$$
\begin{aligned}
(-1)^{j} L_{0} *\left(\Delta+\lambda^{2}\right)^{j} f & =L_{j}\left(P\left(\tau_{-x} f\right)\right)=L_{j}\left(q_{\lambda} P\left(\tau_{-x} f\right)\right) \\
& =\sum_{\nu=0}^{m} a_{\lambda, \nu}(f, x) L_{j}\left(\phi_{\lambda, \nu}\right)=\sum_{\nu=j}^{m} a_{\lambda, \nu}(f, x) H_{j}^{(\nu)}(\lambda) .
\end{aligned}
$$

Además, la matriz triangular

$$
\Lambda=\left(\hat{H}_{j}^{(\nu)}(\lambda) \mid \begin{array}{l}
j=0, \ldots, m \\
\nu=0, \ldots, m
\end{array}\right)
$$


es invertible porque $\operatorname{det} \Lambda=\prod_{j=0}^{m} j !(2 \lambda)^{j} \hat{H}_{0}(\lambda) \neq 0$. Por la fórmula (II.15.5) obtenemos,

$$
\begin{aligned}
{\left[\begin{array}{c}
a_{\lambda, 0}(f, x) \\
a_{\lambda, 1}(f, x) \\
\vdots \\
a_{\lambda, m}(f, x)
\end{array}\right] } & =\left[\begin{array}{c}
L_{0}\left(\tau_{-x} f\right) \\
-L_{0}\left(\tau_{-x}\left(\Delta+\lambda^{2}\right) f\right) \\
\vdots \\
(-1)^{m} L_{0}\left(\tau_{-x}\left(\Delta+\lambda^{2}\right)^{m} f\right)
\end{array}\right] \\
& =\Lambda^{-1}\left[\begin{array}{c}
L_{0} * f \\
-\left(\Delta+\lambda^{2}\right) L_{0} * f \\
\vdots \\
(-1)^{m}\left(\Delta+\lambda^{2}\right)^{m} L_{0} * f
\end{array}\right](x)
\end{aligned}
$$

Si escribimos

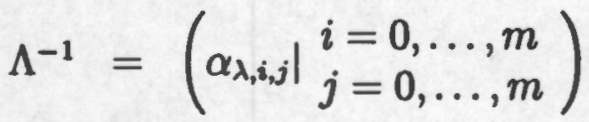

$$
\begin{aligned}
& F_{\lambda, i}=\sum_{j=0}^{m}(-1)^{j} \alpha_{\lambda, i, j}\left(\Delta+\lambda^{2}\right) L_{0}
\end{aligned}
$$

entonces $F_{\lambda, i} \in \mathcal{E}_{K}^{\prime}\left(\mathbb{R}^{n}\right)$ y (II.15.7) implica

$$
a_{\lambda, \nu}(f, \cdot)=F_{\lambda, \nu} * f \in \mathcal{E}_{\lambda}^{(m)}
$$

Por otro lado, (11.15.4) nos dá

$$
q_{\lambda} f(x)=P\left(q_{\lambda}\left(\tau_{-x} f\right)\right)(0)=a_{\lambda, 0}(f, x)
$$

Por lo tanto

$$
q_{\lambda} f=\sum_{j=0}^{m(\lambda)}(-1)^{j} \alpha_{\lambda, 0, j}\left[\left(\Delta+\lambda^{2}\right)^{j} L_{0} * f\right]
$$

El caso $\lambda=0$ es similar. 
Para terminar este capítulo, veamos como la invertibilidad de los operadores $\left.\left(\Delta+z^{2}\right)\right|_{V}, z \notin \sigma(\mathfrak{a})$ nos permite inyectar el espacio $V$ en un subespacio de funciones meromorfas cuyos polos caen en $\sigma(\mathfrak{a})$.

En el teorema II.9 vimos que dado $z \notin \sigma(\mathfrak{a})$, existen $H \in \mathcal{E}_{K}^{\prime}\left(\mathbb{R}^{n}\right)$ y $T \in V_{0}^{\perp}$ tales que

$$
R_{z} f:=\left.\left(\Delta+z^{2}\right)\right|_{v} ^{-1} f=\frac{H * f}{T\left(\phi_{z}\right)} .
$$

De aquí, para cualesquiera $S \in \mathcal{E}^{\prime}\left(\mathbb{R}^{n}\right)$ y $k \in K$ tenemos

$$
S * L_{k} R_{z}(f)=R_{z}\left(S * L_{k} f\right) \text {. }
$$

Se verifica fácilmente que para $\lambda \in \sigma(\mathfrak{a})$ arbitrario,

$$
R_{z}\left(\phi_{\lambda}\right)(x)=\frac{\phi_{\lambda}(x)}{z^{2}-\lambda^{2}}
$$

Definiendo $C_{z}(f)=R_{z} f(0)$, el argumento empleado en (II.14), muestra que $R_{z} f=0$ si y sólo si $f \doteq 0$. En particular $C_{z}(f)=0$ ssi $f=0$. Se cumple:

$$
C_{z}(f):=R_{z} f(0)=P\left(R_{z} f\right)(0)=R_{z}(P f)(0)=C_{z}(P f) .
$$

Por otro lado, los operadores continuos $I_{z}: \mathcal{E}(\mathbb{R}) \rightarrow \mathcal{E}(\mathbb{R}), z \in \mathbb{C}$, definidos por

$$
I_{z} g(x)= \begin{cases}-\frac{1}{z} \int_{0}^{x} g(t) \operatorname{sen} z(t-x) d t, & z \neq 0 \\ -\int_{0}^{x}(t-x) g(t) d t, & z=0\end{cases}
$$

satisfacen $\left(D^{2}-z^{2}\right) I_{z} g=-g$, donde $D=-i \frac{d}{d x}$. Combinando este hecho con la propiedad $\left(\Delta+z^{2}\right) B=-B\left(D^{2}-z^{2}\right)$ obtenemos

$$
\left(\frac{d^{2}}{d r^{2}}+\frac{(n-1)}{r} \frac{d}{d r}+z^{2}\right) B I_{z} g=B g, \quad r \geq 0
$$

La unicidad de soluciones para ecuaciones diferenciales ordinarias nos conduce a

$$
R_{z}(P f)=I B\left(I_{z}(I B)^{-1} P f\right)+C_{z}(f) \phi_{z} ; f \in V, z \notin \sigma(\mathfrak{a})
$$


II.16 Lema. Hay una función suave $\varphi \in C_{0}^{\infty}(\mathbb{R}) \cap W_{0}^{\perp}$ tal que

$$
C_{z}(f)=-\frac{\varphi\left(I_{z}(\mathcal{I B})^{-1} P f\right)}{\hat{\varphi}(z)}, z \notin \sigma(\mathfrak{a}), f \in V .
$$

Demostración. Sean $z_{0} \notin \sigma(\mathfrak{a})$ y $T \in a_{0}$ tales que $\hat{T}\left(z_{0}\right) \neq 0$. Sea $g_{\nu} \in C_{0}^{\infty}\left(\mathbb{R} / \mathbb{Z}_{2}\right)$ con soporte en $[-1 / \nu, 1 / \nu], g_{\nu} \geq 0$ y $\int g_{\nu}=1$ para $\nu=1,2, \ldots$. Puesto que $T * g_{\nu} \in C_{0}^{\infty}\left(\mathbb{R} / \mathbb{Z}_{2}\right)$ y $\left(T * g_{\nu}\right)^{\wedge}$ converge uniformemente en subconjuntos compactos de $\mathbf{C}$, podemos encontrar un $\nu_{0} \in \mathbb{N}$ tal que $\left(T * g_{\nu_{0}}\right)^{\wedge}\left(z_{0}\right) \neq 0$. Hagamos $\varphi=T * g_{\nu_{0}} \in C_{0}^{\infty}\left(\mathbb{R} / \mathbb{Z}_{2}\right) \cap a_{0}$, $L=\left((\mathcal{I B})^{-1}\right)^{\prime} \in V_{0}^{\perp}$ y apliquemos $L$ en ambos miembros de (II.16.5) para obtener

$$
\begin{aligned}
0=L\left(R_{z}(P f)\right) & =L\left(\mathcal{I B I} I_{z}(\mathcal{I B})^{-1} P f\right)+c_{z}(f) L\left(\phi_{z}\right) \\
& =\varphi\left(I_{z}(\mathcal{I B})^{-1} P f\right)+c_{z}(f) \hat{\varphi}(z),
\end{aligned}
$$

por lo tanto

$$
C_{z}(f)=-\frac{\varphi\left(I_{z}(\mathcal{I B})^{-1} P f\right)}{\hat{\varphi}(z)} ; \hat{\varphi}(z) \neq 0, \quad f \in V
$$

Ya que $\varphi \in \mathfrak{a}_{0}$ entonces $\hat{\varphi}(\lambda)=0$ para $\lambda \in \sigma(a)$. Así, solo necesitamos verificar el caso $\hat{\varphi}(a)=0$ cuando $a \notin \sigma(a)$. Sea $\alpha$ el orden de $\hat{\varphi}$ en $a$ y definamos

$$
\varphi_{0}=\varphi, \varphi_{j}=-I_{a} \varphi_{j-1} \in C_{0}^{\infty}\left(\mathbb{R} / \mathbb{Z}_{2}\right), \quad j=1,2, \ldots, \alpha .
$$

Vemos que $\left(D^{2}-a^{2}\right) \varphi_{j}=\varphi_{j-1}, \hat{\varphi}_{j-1}(\xi)=\left(\xi^{2}-a^{2}\right) \hat{\varphi}_{j}(\xi)$ y soporte $\left(\varphi_{j}\right)$ soporte $(\varphi)$ para $j=1,2, \ldots, \alpha$. Además $\varphi_{\alpha} \in a_{0} \cap C_{0}^{\infty}\left(\mathbb{R} / \mathbb{Z}_{2}\right)$. Combinando estas relaciones con (II.16.7) tenemos

$$
\begin{aligned}
C_{z}(f) & =-\frac{\varphi\left(I_{z}(\mathcal{I B})^{-1} P f\right)}{\hat{\varphi}(z)}=-\frac{\left[\left(D^{2}-a^{2}\right) \varphi_{1}\right]\left(I_{z}(\mathcal{I B})^{-1} P f\right)}{\left(z^{2}-a^{2}\right) \hat{\varphi}_{1}(z)} \\
& =-\frac{\varphi_{1}\left(\left(z^{2}-a^{2}\right) I_{z}(\mathcal{I B})^{-1} P f-(\mathcal{I B})^{-1} P f\right)}{\left(z^{2}-a^{2}\right) \hat{\varphi}_{1} \hat{\varphi}_{1}(z)}=-\frac{\varphi_{1}\left(I_{z}(\mathcal{I B})^{-1} P f\right)}{\hat{\varphi}_{1}(z)} \\
& =\cdots=-\frac{\varphi_{\alpha}\left(I_{a}(\mathcal{I B})^{-1} P f\right)}{\hat{\varphi}_{\alpha}(a)}
\end{aligned}
$$


Por lo tanto,

$$
C_{a}(f)=-\frac{\varphi_{\alpha}\left(I_{a}(I B)^{-1} P f\right)}{\hat{\varphi}_{\alpha}(a)}=\lim _{z \rightarrow a} \frac{\varphi\left(I_{z}(I \mathcal{B})^{-1} P f\right)}{\hat{\varphi}(z)} .
$$

Ahora denotemos por $\mathcal{M}(\{\sigma(a), m(\lambda)\})$ al espacio de funciones meromorfas en $\mathbb{C}$ cuyas singularidades caen en $\sigma(a)$ y el orden de éstas en cada $\lambda \in \sigma(\mathfrak{a})$ es $\leq m(\lambda)$. Dotando a éstas de la topología de convergencia uniforme en subconjuntos compactos de $\mathbf{C} \backslash \sigma(\mathfrak{a})$ tenemos:

II.17 Teorema. La aplicación: $V \rightarrow \mathcal{M}(\{\sigma(a), m(\lambda)\}), f \mapsto C_{z}(f)$ es un operador lineal continuo e inyectivo.

Demostración. De los comentarios previos al lema anterior se sigue que la aplicación $C_{z}$ es un operador lineal inyectivo y por ende es suficiente demostrar la continuidad.

Sea $Y \subset \mathbb{C}$ compacto tal que $Y \cap \sigma(\mathfrak{a})$ sea vacío. Escojamos $\varphi \in$ $C_{0}^{\infty}\left(\mathbb{R} / \mathbb{Z}_{2}\right) \cap a_{0}$ como en el lema precedente y asumamos que está soportada en el intervalo $\left[-r_{0}, r_{0}\right]$. A través de la prueba se vió que si para algún $a \notin \sigma(a)$ se tuviera $\hat{\varphi}(a)=0$ entonces podemos encontrar otra función $\varphi_{\alpha}$ con las mismas propiedades pero $\hat{\varphi}_{\alpha}(a) \neq 0$. Ahora, la compacidad de $Y$ nos permite repetir el argumento un número finito de veces así que, sin perder generalidad, podemos suponer que $\hat{\varphi}(z) \neq 0$ para todo $z \in Y$. De aquí

$$
C_{z}(f)=-\frac{\varphi\left(I_{z}(I B)^{-1} P f\right)}{\hat{\varphi}(z)}, \quad z \in Y
$$

La definición de $I_{z}$ en (II.16.3) y un argumento usual nos permiten ver que

$$
\left|C_{z}(f)\right| \leq \frac{2 r_{0}}{\inf |\hat{\varphi}(z)|} \sup _{\substack{|=Y| \leq r_{0} \\ z \in Y}}\left|\frac{\operatorname{sen} z x}{z}\right| \sup _{|x| \leq r_{0}}\left|(\mathcal{I B})^{-1} P f(x)\right| .
$$

Por otro lado, la continuidad de $(I \mathcal{I B})^{-1}$ y $P$ muestran que hay un $N \in \mathbb{N}$ tal que

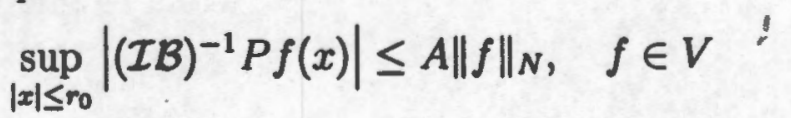


para algún $A \geq 0$. Por lo tanto

$$
\sup _{z \in Y}\left|C_{z} f\right| \leq A(Y)\|f\|_{N} ; \quad z \in Y, \quad f \in V,
$$

donde

$$
A(Y)=\frac{2 r_{0} A}{\inf _{z \in Y}|\hat{\varphi}(z)|} \sup _{\substack{z=\leq r_{0} \\ z \in Y}}\left|\frac{\operatorname{sen} z x}{z}\right|<+\infty
$$

De las últimas relaciones se concluye el teorema.

33 


\section{Aproximación a las soluciones de las ecua- ciones de Weyl y Dirac por promedios esféricos}

III.1 De acuerdo a una fórmula de representación dada en [6] y [18], la solución a la ecuación de Dirac puede escribirse como la esperanza matemática de una variable aleatoria definida en términos del grupo unitario asociado con la ecuación de Weyl. El objetivo principal de este capítulo es obtener el grupo unitario asociado con la ecuación de Weyl como el límite de promedios esféricos adecuados de la condición inicial: Para una función inicial suficientemente regular $\mathbb{C}^{N}$-valuada $\phi(x), x \in$ $\mathbb{R}^{n}$, definimos su promedio esférico

$$
[T(t) \phi](x)=\int_{S^{n-1}}\left(I_{N}+\alpha \cdot \xi\right) \phi(x+n t \xi) d s_{n-1}(\xi),
$$

donde $I_{N}$ es la $N \times N$ matriz identidad,

$$
\alpha \cdot \xi=\sum_{j=1}^{n} \xi_{j} \alpha_{j}, \quad \xi=\left(\xi_{1}, \ldots, \xi_{n}\right) \in S^{n-1}
$$

y $\alpha_{1}, \alpha_{2}, \ldots, \alpha_{n}$ son matrices complejas autoadjuntas de tamaño $N \times N$ con la propiedad anticonmutativa

$$
\alpha_{j} \alpha_{k}+\alpha_{k} \alpha_{j}=2 \delta_{j k}
$$

La ecuación de Weyl sin potencial es la e.d.d.p. (ecuación diferencial en derivadas parciales) dada por

$$
\begin{aligned}
& \frac{\partial \psi}{\partial t}=\alpha \cdot \nabla \psi=\sum_{j=1}^{n} \alpha_{j} \frac{\partial \psi}{\partial x_{j}} \\
& \psi=\psi(t, x), t>0 \text { y } \psi(0, x)=\phi(x)
\end{aligned}
$$

Por la teoría espectral clásica en espacios de Hilbert, la solución de esta e.d.d.p. se representa como el semigrupo unitario

$$
\psi(t, x)=\left(e^{t a \cdot \nabla} \phi\right)(x), \phi \in \operatorname{Dom}(\alpha \cdot \nabla), \quad:
$$


donde

$$
\alpha \cdot \nabla=\sum_{j=1}^{n} \alpha_{j} \frac{\partial}{\partial x_{j}}
$$

y $\operatorname{Dom}(\alpha \cdot \nabla)=\left[W_{2,1}\left(\mathbb{R}^{n}\right)\right]^{N}=N$ copias del espacio de Sobolev de orden 1 en $L^{2}\left(\mathbb{R}^{n}\right)$. De esta manera, debemos mostrar

$$
\lim _{m \rightarrow \infty} T(t / m)^{m} \phi=e^{t \alpha \cdot \nabla} \phi \quad \text { en, } L^{2}\left(\mathbb{R}^{n}\right)^{N} .
$$

La combinación de estos promedios con la fórmula probabilística (III.7.2) (ver [20]) nos da una representación de la solución a la ecuación de Dirac como la esperanza del límite de tales promedios esféricos. La representaciones obtenidas de esta manera para las soluciones de tales ecuaciones se pueden considerar como integrales sobre un espacio de trayectorias en $S^{n-1}$ de dimensión infinita, definidas como límite de integrales sobre espacios finito-dimensionales que pueden interpretarse bien como espacios de trayectorias poligonales. La velocidad de las trayectorias involucradas, igual a la dimensión espacial $n$, no influye para estimar la norma del operador $T(t)$ pero sí para conseguir que $\left.\frac{d T}{d t}\right|_{t=0}$ sea igual al Hamiltoniano de Weyl.

III.2 La ecuación de Dirac es una e.d.d.p de la forma

$$
-i \frac{\partial \psi(t, x)}{\partial t}=\left[\sum_{j=1}^{n} \alpha_{j}\left(-i \frac{\partial}{\partial x_{j}}+e A_{j}(x)\right)+e V+m \beta\right] \psi(t, x),
$$

donde $\psi$ es una función de onda en un espacio de Hilbert adecuado $\mathbb{H}$, $A_{j}(j=1,2, \ldots, n)$ y $V$ son funciones real valuadas (los potenciales); $\alpha_{j}(j=1,2, \ldots, n)$ y $\alpha_{n+1}=\beta$ son variables anticonmutativas que satisfacen

$$
\alpha_{j} \alpha_{k}+\alpha_{k} \alpha_{j}=2 \delta_{j, k} \text { para } j, k=1,2, \ldots, n .
$$

La ecuación está escrita en unidades para las cuales $c=\hbar=1, m$ es la masa en reposo y $e$ es la carga.

Las variables anticonmutativas $\alpha_{j}$ pueden representarse por medio de las matrices complejas de un cierto tamaño $N \times N$ y el espacio de 
Hilbert puede escogerse como el espacio

$$
\mathbb{H}=L^{2}\left(\mathbb{R}^{n}\right)^{N}=\left\{\phi=\left(\phi_{1}, \ldots, \phi_{N}\right): \phi_{j} \in L^{2}\left(\mathbb{R}^{n}\right), 1 \leq j \leq n\right\},
$$

dotado con el producto interno de la suma directa ortogonal

$$
\langle\phi \mid \psi\rangle:=\sum_{\ell=1}^{N} \int \phi_{\ell}(x) \bar{\psi}_{\ell}(x) d x
$$

Por simplicidad consideraremos aquí sólo la ecuación de Dirac Libre, i.e., $V(x)=A_{j}(x)=0$ para $x \in \mathbb{R}^{n}$ y $1 \leq j \leq n$. Esta restricción no es esencial porque los potenciales no nulos se pueden tratar mediante la adición de una perturbación. Tal método se postpone para el próximo capítulo.

Denotemos por $W_{2,1}\left(\mathbb{R}^{n}\right)$ el espacio de Sobolev de orden 1

$W_{2,1}\left(\mathbb{R}^{n}\right)=\left\{f \in L^{2}\left(\mathbb{R}^{n}\right) \mid f\right.$ es dérivable y $\left.\frac{\partial f}{\partial x_{j}} \in L^{2}\left(\mathbb{R}^{n}\right), 1 \leq j \leq n\right\}$,

con el producto interno $\langle f \mid g\rangle_{2,1}=\langle f \mid g\rangle_{L^{2}\left(\mathbb{R}^{n}\right)}+\langle\nabla f \mid \nabla g\rangle_{L^{2}\left(\mathbb{R}^{n}\right)^{N}}$.

Como es usual, $\mathcal{B}(\mathbb{H})$ denota el espacio de operadores acotados en $\mathbb{H}$ con la norma de operadores

$$
\|s\|_{\mathcal{B}(\mathbf{H})}:=\sup _{\|f\| \leq 1}\|s f\|_{\mathbb{H}}
$$

En el capítulo I se definió la transformada de Fourier de una distribución arbitraria. Se puede verificar que el operador $T(t)$ se expresa mediante una matriz con elementos en $\mathcal{E}^{\prime}\left(\mathbb{R}^{n}\right)$ sin embargo tal representación dificulta nuestras estimaciones. En el resto de nuestro trabajo adoptamos la convención

$$
\hat{T}(t)(z):=\int_{S^{n-1}}(I+\alpha \cdot \xi) e^{i n t<z, \xi>} d s_{n-1}(\xi)
$$

Tal abuso de notación se hará únicamernte en este casa y su justificación reside en la reducción notacional de las estimaciones sin alterarlas. El siguiente resultado será de crucial importancia en nuestro trabajo. 
III.3 Teorema. Si \| $\|$ denota la norma euclideana usual en $\mathbb{C}^{n}$, entonces

(a) Para cualesquier $u \in \mathbb{C}^{n}$ se tiene

$$
\sup _{\substack{t \in \mathbb{R}^{n} \\ z \in R^{n}}}\|\hat{T}(t)(\mathfrak{z}) u\| \leq\|u\|
$$

(b) $\|\hat{T}(t)(3)\|_{\mathcal{B}(\mathrm{H})} \leq 1$

Demostración. Como se vió en el capítulo II, la función

$$
\varphi(r)=\int_{-1}^{1}\left(1-s^{2}\right)^{\frac{n-3}{2}} e^{-i r s} d s=\int_{-1}^{1}\left(1-s^{2}\right)^{\frac{n-3}{2}} \cos (r s) d s, r>0
$$

satisface

$$
\varphi^{\prime \prime}(r)+\frac{(n-1)}{r} \varphi^{\prime}(r)+\varphi(r)=0, \quad \varphi(0)=0, \quad \lim _{r \rightarrow 0} \frac{\varphi^{\prime}(r)}{r}=1,
$$

$\varphi$ es real valuada y

$$
\varphi\left(n t\left\|_{\mathfrak{z}}\right\|\right)=\int_{\mathbf{a}^{n-1}} e^{i n t<\}, \xi>} d s_{n-1}(\xi), \quad z \in \mathbb{R}^{n}
$$

Por lo tanto (III.3.2) y (III.2.6) nos dan

$$
\begin{aligned}
\hat{T}(t)(\mathfrak{z}) & =\varphi\left(n t\left\|_{3}\right\|\right) I_{N}+\frac{1}{i n t} \alpha \cdot \nabla_{3} \varphi\left(n t\left\|_{3}\right\|\right) \\
& =\varphi\left(n t\left\|_{3}\right\|\right) I_{N}-i \frac{\alpha \cdot \mathfrak{z}}{\|z\|} \varphi^{\prime}\left(n t\left\|_{3}\right\|_{3}\right)_{3} \in \mathbb{R}^{n}
\end{aligned}
$$

Aplicando esta relación a $u \in \mathbb{C}^{n}$ obtenemos

$$
\begin{aligned}
& \|\hat{T}(t)(z) u\|^{2}=\varphi\left(n t\left\|_{3}\right\|\right)^{2}\|u\|^{2}+\frac{i \varphi\left(n t\left\|_{3}\right\|\right)}{\|z\|} \sum_{\ell=1}^{n} z_{\ell}\left\langle u, \alpha_{\ell} u\right\rangle \\
& \frac{-i \varphi\left(n t\left\|_{3}\right\|\right)}{\left\|_{3}\right\|} \sum_{\ell=1}^{n} z_{\ell}\left\langle\alpha_{\ell} u, u\right\rangle+\frac{\varphi^{\prime}\left(n t\left\|_{3}\right\|\right)}{\left\|_{3}\right\|^{2}} \sum_{\ell, k=1}^{n} z_{\ell} z_{k}\left\{\alpha_{\ell} u, \alpha_{k} u\right\rangle
\end{aligned}
$$


ya que cada $\alpha_{\ell}$ es autoadjunta y $\alpha_{k} \alpha_{\ell}=-\alpha_{\ell} \alpha_{k}$ para $k \neq \ell$ entonces la igualdad precedente se reduce a:

$$
\begin{aligned}
\|\hat{T}(t)(z) u\|^{2} & =\varphi\left(n t\left\|_{z}\right\|\right)^{2}\|u\|^{2}+\frac{\varphi^{\prime}\left(n t\left\|_{3}\right\|\right)}{\|z\|^{2}} \sum_{\ell=1}^{n} z_{\ell}^{2}\|u\|^{2} \\
& =\left[\varphi\left(n t\left\|_{z}\right\|\right)^{2}+\varphi^{\prime}\left(n t\left\|_{3}\right\|\right)^{2}\right]\|u\|^{2}
\end{aligned}
$$

Definiendo $g(s)=\varphi(s)^{2}+\left[\varphi^{\prime}(s)\right]^{2}, s \geq 0$, la relación (III.3.1) nos lleva a

$$
g^{\prime}(s)=2 \varphi^{\prime}(s)\left[\varphi(s)+\varphi^{\prime \prime}(s)\right]=-\frac{2(n-1)}{s}\left[\varphi^{\prime}(s)\right]^{2} \leq 0, \quad s \geq 0, \quad n \geq 1
$$

i.e., $g$ es monótona decreciente. De aqú́ $g(s) \leq g(0)=1, s \geq 0$. Consecuentemente (III.3.4) implica que

$$
\sup _{\substack{i \in \mathbb{R} \\ z \in \mathbb{R}^{n}}}\|\hat{T}(t)(\xi) u\|^{2} \leq g(0)\|u\|^{2}=\|u\|^{2} .
$$

Esto demuestra (a). Para obtener (b) observemos que (a) afirma que

$$
\sup _{z \in \mathbb{R}^{n}, t \in \mathbb{R}}\|\hat{T}(t)(z)\|_{\mathcal{B}\left(\mathbb{C}^{N}\right)}:=\sup _{\substack{\|u\|=1 \\ z \in \mathbb{R}^{n}, t \in \mathbb{R}}}\|\hat{T}(t)(z) u\| \leq 1,
$$

Si $\phi \in L^{2}\left(\mathbb{R}^{n}\right)^{N}$, el Teorema de Plancherel asegura que la transformada de Fourier actuando en este espacio es una isometría. Puesto que $[T(t) \phi]^{\wedge}(z)=\hat{T}(t)(\xi) \hat{\phi}(z)$, entonces III.3.5 nos conduce a

$$
\left\|[T(t) \phi]^{\wedge}(z)\right\|^{2}=\|\hat{T}(t)(z) \hat{\phi}(z)\|^{2} \leq\|\hat{\phi}(z)\|^{2}, z \in \mathbb{R}^{n} \text { y } \phi \in \mathbb{H} .
$$

Integrando sobre todo $\mathbb{R}^{n}$ concluímos que para todo $t>0$,

$$
\|[T(t) \phi]\|_{\mathbf{H}}=\left\|[T(t) \phi]^{\wedge}\right\|_{\mathbf{H}} \leq\|\hat{\phi}\|_{\mathbf{H}}=\|\phi\|_{\mathbf{H}}, \phi \in \mathbb{H} .
$$

y la demostración está completa. 
III.4 Lema. $\left.\frac{d T}{d t}\right|_{t=0}=\alpha \cdot \nabla$ en $\operatorname{Dom}(\alpha \cdot \nabla)=W_{2,1}\left(\mathbb{R}^{n}\right)^{N}$.

Demostración. Si $\phi \in \mathcal{D}(\alpha \cdot \nabla)$, el teorema de derivación bajo el signo de integral se aplica para obtener

$$
\begin{aligned}
\lim _{h \rightarrow 0} \frac{[T(t+h) \phi](x)-[T(t) \phi](x)}{h}=\frac{d}{d t}[T(t) \phi](x)= \\
=\int_{S^{n-1}}(I+\alpha \cdot \xi)<\nabla \phi(x+n t \xi), \xi>d_{s_{n-1}}(\xi) .
\end{aligned}
$$

Ya que la permutación de coordenadas y el cambio de signo en cualquiera de ellas son transformaciones ortogonales en $\mathbb{R}^{n}$, la $K$-invariancia de $d s_{n-1}$ nos da

$$
\int_{S^{n-1}} \xi_{j} d s_{n-1}(\xi)=0 \text { y } \int_{S^{n-1}} \xi_{j} \xi_{k} d s_{n-1}(\xi)=\frac{\delta_{j k}}{n}, \text { para } j, k=1,2, \ldots, n \text {. }
$$

Así, en $t=0$ obtenemos $T(0)=I$ y

$$
\begin{aligned}
\frac{d}{d t}[T(t) \phi](x)= & n \sum_{j=1}^{n}\left(\int_{S^{n-1}} \xi_{j} d s_{n-1}(\xi)\right) \frac{\partial \phi}{\partial x_{j}}(x) \\
& +n \sum_{j \neq k}\left(\int_{S^{n-1}} \xi_{j} \xi_{k} d s_{n-1}(\xi)\right) \alpha_{j} \frac{\partial \phi}{\partial x_{k}}(x) \\
& +n \sum_{j=1}^{n}\left(\int_{S^{n-1}} \xi_{j}^{2} d s_{n-1}(\xi)\right) \alpha_{j} \frac{\partial \phi}{\partial x_{j}}(x) \\
= & \alpha \cdot \nabla \phi(x) .
\end{aligned}
$$

III.5 Observación. Como ya se mencionó $e^{t \alpha \cdot \nabla}, t \geq 0$ es un semigrupo continuo de operadores unitarios cuyo generador infinitesimal es $\alpha \cdot \nabla$ y para $\phi \in \operatorname{Dom}(\alpha \cdot \nabla), e^{t \alpha \cdot \nabla} \phi$ es la solución de la ecuación de Weyl (III.1.4). Lo anterior es consecuencia inmediata de la teoría espectral clásica en espacios de Hilbert, sin embargo, ésto se puede argumentar de manera sencilla: En $L^{2}\left(\mathbb{R}^{n}\right)^{N}$ defínase

$$
[\tilde{G}(t) \hat{\phi}](\mathfrak{z}):=\left\{\begin{array}{ll}
{\left[\cos \left(t\left\|_{\mathfrak{z}}\right\|\right)-i \frac{\operatorname{sen}\left(t\left\|_{\mathfrak{z}}\right\|\right)}{\|\mathfrak{}\|} \alpha \cdot \mathfrak{z} \mid \hat{\phi}(\mathfrak{z}),\right.} & 0 \neq \mathfrak{z} \in \mathbb{R}^{n}, \\
\hat{\phi}(\mathfrak{z}) & \mathfrak{z}=0 .
\end{array}, t \in \mathbb{R} .\right.
$$


Procediendo como en el teorema II.3 se verifica fácilmente que para cualesquiera $t \in \mathbb{R}$ y $\boldsymbol{z}, u \in \mathbb{R}^{n}$

$$
\left\|\left[\cos (t|z|)-i \frac{\operatorname{sen}\left(t\left\|_{3}\right\|\right)}{\|z\|} \alpha \cdot z\right] u\right\|=\|u\|
$$

después, integrando sobre $\mathbf{z} \in \mathbb{R}^{n}$ obtenemos $\|\tilde{G}(t) \hat{\phi}\|_{\mathbf{H}}=\|\hat{\phi}\|_{\mathbb{H}}$. Observando que $\tilde{G}\left(t_{1}+t_{2}\right)=\tilde{G}\left(t_{1}\right) \tilde{G}\left(t_{2}\right)$ se sigue sin más que $\tilde{G}(t), t \in \mathbb{R}$, es un semigrupo continuo de operadores unitarios. Para obtener el generador infinitesimal de $\tilde{G}$, tomemos en cuenta la identidad

$$
(\alpha \cdot z)^{2}=\left(\sum z_{j} \alpha_{j}\right)^{2}=z_{1}^{2}+z_{2}^{2}+\cdots+z_{n}^{2} .
$$

Para $z \in \mathbb{R}^{n}$, la diferenciabilidad de $\tilde{G}$ nos dará

$$
\frac{d}{d t} \tilde{G}(t) \hat{\phi}(\mathfrak{\jmath})=-i \alpha \cdot z \tilde{G}(t) \hat{\phi}(z)=\tilde{G}(t)(-i \alpha \cdot z \hat{\phi}(\mathfrak{z})), \quad z \in \mathbb{R}^{n}
$$

Es inmediato de (III.5.3) que la condición necesaria y suficiente para que $\frac{d}{d t} \tilde{G}(t) \phi \in L^{2}\left(\mathbb{R}^{n}\right)^{N}$ es que $z_{\ell} \hat{\phi}(\mathfrak{z}) \in L^{2}\left(\mathbb{R}^{n}\right)^{N}$ para $\ell=1,2, \ldots, n$. De esta manera, el teorema de Plancherel asegura que para $t \in \mathbb{R}$ hay un operador unitario único $G(t)$ definido en $L^{2}\left(\mathbb{R}^{n}\right)^{N}$ tal que $[G(t) \phi]^{\wedge}=$ $\tilde{G}(t) \hat{\phi}, \phi \in L^{2}\left(\mathbb{R}^{n}\right)^{N}$.

La propiedad de semigrupo de $G$ se hereda de $\tilde{G}$. En particular $G$ depende continuamente de $t$. De (III.5.3) se deduce que

$$
\frac{d}{d t} G(t) \phi=\alpha \cdot \nabla G(t) \phi=G(t) \alpha \cdot \nabla \phi, \phi \in W_{1,2}\left(\mathbb{R}^{n}\right)^{N} \text { y } G(0) \phi=\phi .
$$

La definición de $\tilde{G}(t)$ nos permite extraer mayor información que no puede obtenerse con la teoría espectral clásica. En efecto, notemos que las distribuciones $u_{1}, u_{2}, \in \mathcal{E}^{\prime}\left(\mathbb{R} / \mathbb{Z}_{2}\right)$ dadas por

$$
u_{1}(f)=\frac{f(t)+f(-t)}{2}, \quad u_{2}(f)=\frac{1}{2} \int_{-t}^{t} f(\tau) d \tau
$$

satisfacen

$$
\hat{u}_{1}(\lambda)=\cos (t \lambda) \quad \text { y } \quad \hat{u}_{2}(\lambda)=\frac{\operatorname{sen}(t \lambda)}{\lambda}, \quad \dot{\lambda} \in \mathbb{C}
$$


por lo tanto, (II.7.3) y (II.7.4) nos dan

$$
[\tilde{G}(t) \hat{\phi}](\mathfrak{z})=\left\{\left[(\mathcal{I B})^{\prime-1} u_{1}+\left((\mathcal{L B})^{\prime-1} u_{2}\right) * \alpha \cdot \nabla\right] * \phi\right\}^{\wedge}(\mathfrak{z})
$$

i.e. $e^{t a \cdot \nabla}$ es un operador definido por la convolución de una matriz $N \times N$ con coeficientes en $\mathcal{E}^{\prime}\left(\mathbb{R}^{n}\right)$.

Tenemos el resultado principal de este capítulo:

III.6 Teorema. Para cada $\phi \in W_{2,1}\left(\mathbb{R}^{n}\right)^{N}=\operatorname{Dom}(\alpha \cdot \nabla)$ se cumple

$$
\lim _{m \rightarrow \infty}\left[T(t / m)^{m} \phi\right](x)=e^{t a \cdot \nabla} \phi(x) .
$$

Demostración. Seguiremos el método de Nelson para la fórmula del producto de Trotter.

Dada $\phi \in \mathcal{D}(\alpha \cdot \nabla)$ tenemos

$$
\begin{aligned}
\lim _{t \rightarrow 0} \frac{1}{t}\left[T(t)-e^{t \alpha \cdot \nabla}\right] & =\lim _{t \rightarrow 0}\left[\frac{1}{t}(T(t) \phi-\phi)-\frac{1}{t}\left(e^{t \alpha \cdot \nabla} \phi-\phi\right]\right. \\
& =\alpha \cdot \nabla \phi-\alpha \cdot \nabla \phi=0 .
\end{aligned}
$$

Se sigue que $\left\{\frac{1}{t}\left[T(t)-e^{t a \cdot \nabla} \mid \phi: t>0\right\}\right.$ es un subconjunto acotado de IH. El teorema de Banach-Steinhaus aplicado a los espacios de Banach $\mathbb{H}$ y $\mathcal{D}(\alpha \cdot \nabla)$ asegura la existencia de una constante $c$ tal que

$$
\sup _{t>0}\left\|\frac{1}{t}\left(T(t)-e^{t \alpha \cdot \nabla}\right) \phi\right\|_{\mathrm{B}} \leq c\|\phi\|_{2,1}, \phi \in \mathcal{D}(\alpha \cdot \nabla),
$$

donde \|\|$_{2,1}$ denota la norma en $W_{2,1}\left(\mathbb{R}^{n}\right)^{N}$.

Esto es, la familia de operadores

$$
\gamma(t)=\frac{1}{t}\left(T(t)-e^{t \alpha \cdot \nabla}\right): \mathcal{D}(\alpha \cdot \nabla) \rightarrow \mathbb{H}, t>0,
$$

es un conjunto uniformemente acotado de operadores que tiende fuertemente a cero cuando $t \rightarrow 0$. 
Sean $\epsilon>0$ y $t>0$. Es suficiente demostrar que hay un $m_{0} \in \mathbb{N}$ tal que

$$
\sup _{0 \leq \tau \leq t}\left\|\left(T(\tau / m)^{m}-e^{\tau \alpha \cdot \nabla}\right) \phi\right\|_{\mathrm{H}}<t \epsilon, \quad m \geq m_{0} .
$$

Para obtenerlo, primero veamos que

$$
\lim _{\tau \rightarrow 0} \sup _{\varphi \in \mathcal{A}}\|\gamma(\tau) \varphi\|_{\mathrm{H}}=0 \text { para } \mathcal{A} \subset \operatorname{Dom}(\alpha \cdot \nabla), \mathcal{A} \text { compacto. }
$$

Efectivamente, escojamos $\varphi_{1}, \ldots, \varphi_{r} \in \mathcal{A}$ con la siguiente propiedad:

Para $\varphi \in \mathcal{A}$ hay un $\varphi_{j}$ tal que $\left\|\varphi-\varphi_{j}\right\|_{2,1}<\epsilon / 2(1+c)$. Sea $\theta>0$ tal que

$$
\sup _{0<\tau \leq \theta}\left\|\gamma(\tau) \varphi_{j}\right\|_{\mathrm{H}}<\frac{\epsilon}{2} \text { para } j=1,2, \ldots, r .
$$

Aplicando (III.6.1) obtenemos

$$
\begin{aligned}
\|\gamma(\tau) \varphi\|_{\mathrm{H}} & \leq\left\|\gamma(\tau)\left(\varphi-\varphi_{j}\right)\right\|_{\mathrm{H}}+\left\|\gamma(\tau) \varphi_{j}\right\|_{\mathrm{H}} \leq C\left\|\varphi-\varphi_{j}\right\|_{2,1}+\epsilon / 2 \\
& \leq C \frac{\epsilon}{2(1+C)}+\epsilon / 2<\epsilon \text { para } 0 \leq \tau \leq \theta \text { y } \varphi \in \lambda .
\end{aligned}
$$

De aquí se sigue (III.6.3).

Ahora, tomando en cuenta que

$$
\|f\|_{2,1} \leq \text { cte } \int_{\mathbb{R}^{n}}(1+\|z\|)^{2}\|\hat{f}(z)\|^{2} d z
$$

entonces la definición de $\tilde{G}$, (III.5.1) y el Teorema de Convergencia Dominada de Lebesgue se emplean para mostrar la continuidad de la función: $[0, t] \rightarrow \mathcal{D}(\alpha \cdot \nabla), s \mapsto e^{\alpha a \cdot \nabla} \phi$. Por lo tanto, aplicando (III.6.3) al subconjunto compacto $\mathcal{A}=\left\{e^{s \alpha \cdot \nabla} \phi: 0 \leq s \leq t\right\} \subset \operatorname{Dom}(\alpha \cdot \nabla)$, se verifica: Existe $m_{0} \in \mathbb{N}$ tal que

$$
\sup _{\substack{0 \leq 0 \leq t \\ m \geq m_{0}}}\left\|\frac{t}{m}\left[T(t / m)-e^{\frac{t}{m} \alpha \cdot \nabla}\right] e^{s \alpha \cdot \nabla} \phi\right\|_{H}<\epsilon
$$

Por otro lado se tiene

$$
T(t / m)^{m}-e^{t \alpha \cdot \nabla}=\sum_{\ell=0}^{m-1} T(t / m)^{\ell}\left[T(t / m)-e^{\frac{\ell}{m} \alpha \cdot \nabla}\right] e^{\frac{m+\ell-1}{m} t \alpha \cdot \nabla}
$$


Por el teorema III.3 y las relaciones (III.6.4), (III.6.5), obtenemos la estimación

$$
\begin{aligned}
\left\|\left[T(\tau / m)^{m}-e^{\tau \alpha \cdot \nabla}\right] \phi\right\|_{\mathrm{B}} \leq \sum_{\ell=0}^{m-1}\left\|\left[T(\tau / m)-e^{\frac{\tau}{m} \alpha \cdot \nabla}\right] e^{\frac{m-\ell-1}{m} \tau \alpha \cdot \nabla} \phi\right\|_{\mathbf{H}} \\
\leq \sum_{\ell=0}^{m-1} \frac{\tau}{m}\left\|\frac{1}{(\tau / m)}\left[T(\tau / m)-e^{\frac{\tau}{m} \alpha \cdot \nabla}\right] e^{\frac{m-\ell-1}{m} \tau \alpha \cdot \nabla} \phi\right\|_{\mathbf{H}} \\
\leq \sum_{\ell=0}^{m-1} \frac{\tau}{m} \epsilon=\tau \epsilon \leq t \epsilon, m \geq m_{0} .
\end{aligned}
$$

De este modo (III.6.2) está demostrado y el teorema concluye.

Como se dijo antes la ecuación de Dirac libre viene dada por

$$
-i \frac{\partial \psi}{\partial t}(t, x)=(-i \alpha \cdot \nabla+m \beta) \psi(t, x), \quad \psi(0, x)=\phi(x) .
$$

En [Que- ] se demostró que la ecuación de Dirac libre se puede representar en la forma

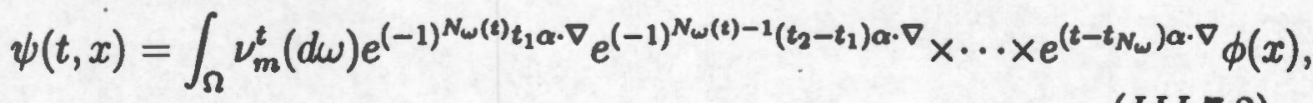

donde $(\Omega, \mathbb{F}, P)$ es el espacio de probabilidad asociado con un proceso de conteo de Poisson con intensidad $m$ (la masa en reposo), $0<t_{1}<$ $\ldots<t_{N_{\omega}}<t$ son los tiempos de salto de este proceso y $\nu_{m}^{t}$ es la medida valuada en las matrices complejas $N \times N$ tal que

$$
d \nu_{m}^{t}=e^{m t}(i \beta)^{N_{\omega}(t)} d P .
$$

Ahora damos las aproximaciones para la solución de la ecuación de Dirac:

III.7 Teorema. Sea $\psi$ la solución de la ecuación de Dirac libre (III.7.1) con condición inicial $\phi \in W_{2,1}\left(\mathbb{R}^{n}\right)^{N}$, entonces

$$
\psi(t, x)=\lim _{k \rightarrow \infty} \int_{\omega \in \Omega} \nu_{m}^{t}(d \omega)\left(\left[T\left(\frac{t_{1}}{k}\right)^{k} T\left(\frac{t_{2}-t_{1}}{k}\right)^{k} \therefore T\left(\frac{t-t_{N_{\omega}}}{k}\right)^{k}\right] \phi(x)\right)
$$


Demostración. Para $\omega \in \Omega$ y $k \in \mathbb{N}$ sean

$$
\begin{aligned}
& \phi_{k}(t, x, \omega)=\left[\dot{T}\left(t_{1} / k\right)^{k} T\left(\frac{t_{2}-t_{1}}{k}\right)^{k} \cdots T\left(\frac{t-t_{N_{\omega}}}{k}\right)^{k}\right] \phi(x) \mathrm{y} \\
& \psi_{k}(t, x)=\int_{\Omega} \nu_{m}^{t}(d \omega) \phi_{k}(t, x, \omega) .
\end{aligned}
$$

Por el teorema precedente, para cada $\omega \in \Omega$

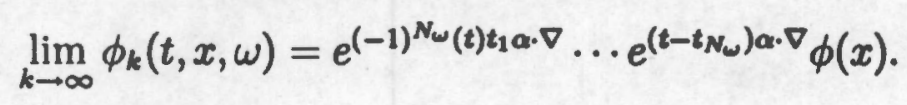

Por el teorema III.3

$$
\left\|T\left(t_{1} / k\right)^{k} \ldots T\left(\frac{t-t_{N_{w}}}{k}\right)^{k}\right\|_{B(\mathrm{H})} \leq 1
$$

Como se vió en III.5 el semigrupo es unitario, por lo tanto, el segundo miembro de (III.7.5) es una función acotada de $\omega$. De (III.7.6) se deduce que cada $\phi_{k}(t, x, \cdot)$ lo es también. Por (III.7.3) la variación total de $\nu_{m}^{t}$ es

$$
\int_{\Omega}\left|\nu_{m}^{t}(d \omega)\right| \leq \int_{\Omega} e^{m t}\left\|(i \beta)^{N_{\omega} t}\right\| P(d \omega)=e^{m t}<+\infty .
$$

De aquí se tiene que toda función acotada en $\omega$ es $\nu_{m}^{t}$-integrable. Luego, aplicando el Teorema de Convergencia Dominada y la representación (III.7.2) se sigue que la sucesión $\psi_{k}(t, x)$ converge puntualmente a $\psi(t, x)$. Empleando el teorema de Fubini, la relación (III.7.2) y ya que la convergencia en (III.7.5) es en $\mathbb{H}$ con respecto a $(t, x)$, entonces la deseatla convergencia en $\mathbb{H}$ se tiene. 


\section{Convergencia suave de las aproximaciones esféricas}

IV.1 Denotemos por $\mathcal{M}\left(m_{1}, m_{2} ; \mathbf{C}\right)$ el espacio de matrices $m_{1} \times m_{2}$ sobre $\mathbb{C}$ provisto con la norma de operadores \|\|$_{\mathcal{M}}$. Cuando $m_{1}=m_{2}$ sólo escribiremos $\mathcal{M}\left(m_{1} ; \mathbb{C}\right)$. Si, $p \geq 1$ y $q \geq 0$, consideramos el espacio $W^{p, q}\left(\mathbb{R}^{n}, \mathbb{C}^{N}\right)=W^{p, q}\left(\mathbb{R}^{n}\right)^{N}$ consistente de todas las funciones medibles $f: \mathbb{R}^{n} \rightarrow \mathbb{C}^{N}$ que satisfacen

$$
\|f\|_{q, p}^{q}:=\int_{\mathbb{R}^{n}}(1+\|y\|)^{q}\|f(y)\|^{p} d y<+\infty
$$

La definición de norma y seminormas se extienden de manera natural a los espacios $W^{p, q}\left(\mathbb{R}^{n}, \mathcal{M}\left(m_{1}, m_{2} ; \mathbb{C}\right)\right)$ y $C^{\infty}\left(\mathbb{R}^{n}, \mathcal{M}\left(m_{1}, m_{2} ; \mathbb{C}\right)\right)$ respectivamente. Para $\phi \in \mathcal{E}\left(\mathbb{R}^{n}\right)^{N}$, la familia de operadores $\{T(t)\}_{t \in \mathbb{R}}$,

$$
[T(t) \phi](x)=\int_{S^{n-1}}(I+\alpha \cdot \xi) \phi(x+n t \xi) d s_{n-1}(\xi) ; \quad t \in \mathbb{R},
$$

define una curva suave cuya $k$-ésima derivada $D^{k} T \in C^{\infty}\left(\mathbb{R}, \mathcal{B}\left(\mathcal{E}\left(\mathbb{R}^{n}\right)^{N}\right)\right)$ es

$$
\left\{\left[D^{k} T(t)\right] \phi\right\}(x)=n^{k} \int_{S^{n-1}}(I+\alpha \cdot \xi)\left(\sum_{j=1}^{n} \xi_{j} \frac{\partial}{\partial x_{j}}\right)^{k} \phi(x+n t \xi) d s_{n-1}(\xi),
$$

donde $\mathcal{B}\left(\mathcal{E}\left(\mathbb{R}^{n}\right)^{N}\right)$ denota el espacio de operadores continuos en $\mathcal{E}\left(\mathbb{R}^{n}\right)^{N}$ provisto de la $\beta$-topología definida por las seminormas

$$
\sup _{\phi \in \mathcal{A}}\|L \phi\|_{\ell} ; \mathcal{A} \subset \mathcal{E}\left(\mathbb{R}^{n}\right)^{N} \text { acotado y } \ell \in \mathbb{N} \text {. }
$$

Dada $B \in C^{\infty}\left(\mathbb{R}^{n}, \mathcal{M}(N, \mathbb{C})\right)$ definimos la curva $Q \in C^{\infty}\left(\mathbb{R}, \mathcal{B}\left(\mathcal{E}\left(\mathbb{R}^{n}\right)^{N}\right)\right)$, $[Q(t) \phi](x)=[T(t) \phi](x)+t B(x) \phi(x)=\{[T(t)+t B] \phi\}(x) ; t \in \mathbb{R}, x \in \mathbb{R}^{n}$.

El objetivo de este capítulo es:

(a) Obtener la exiśtencia y unicidad del problema de Cauchy

$$
\frac{\partial \psi}{\partial t}=(\alpha \cdot \nabla+B) \psi ; \psi(0)=I, \quad \psi \in C^{\infty}\left(\mathbb{R}^{\prime}, \mathcal{B}\left(\mathcal{E}\left(\mathbb{R}^{n}\right)^{N}\right)\right) \text {. }
$$


(b) Para cada $j \in \mathbb{N}$ y $\ell \in \mathbb{N}$

$$
\begin{gathered}
\lim _{m \rightarrow \infty}\left[\sup _{\substack{|t| \leq j \\
|=| \leq \ell^{\prime}|\mathbf{a}| \leq \ell}}\left\|\partial_{x}^{a}\left\{\left[Q(t / m)^{m} \phi\right](x)-[\psi(t) \phi](x)\right\}\right\|\right]=0 \\
\left.\lim _{m \rightarrow \infty}\left[\sup _{\substack{|t| \leq j \\
|=| \leq \ell^{\prime}|\mathbf{a}| \leq \ell}} \| \frac{d}{d t} \partial_{x}^{a}\left\{\left[Q(t / m)^{m} \phi\right](x)-\mid \psi(t) \phi\right](x)\right\} \|\right]=0, \\
\text { donde } \mathbf{a}=\left(a_{1}, \ldots, a_{n}\right) \text { es un multíndice y }|\mathbf{a}|=a_{1}+\ldots+a_{n}
\end{gathered}
$$

El método presentado aquí es esencialmente el método de Nelson combinado con la fórmula de Taylor. Sean $M \in W^{q, 1}\left(\mathbb{R}^{n}, \mathbb{C}^{N}\right)$ y $\mathbf{b}=$ $\left(b_{1}, \ldots, b_{n}\right)$ un multiíndice de altura $|\mathrm{b}|=q$. Si escribimos

$$
\left\|z^{b} M\right\|_{L^{1}}:=\int_{\mathbb{R}^{n}}\left\|z^{b} M(z)\right\|_{\mathcal{M}} d z
$$

donde $z^{\mathrm{b}}=z_{1}^{b_{1}} z_{2}^{b_{2}} \ldots z_{n}^{b_{n}}$ entonces

IV.2 Lema. Dados $A_{1} \in W^{q, 1}\left(\mathbb{R}^{n}, \mathcal{M}\left(N_{1}, N_{2} ; \mathbb{C}\right)\right), A_{2} \in W^{q, p}\left(\mathbb{R}^{n}, \mathcal{M}\left(N_{2}, N_{3} ; \mathbb{C}\right)\right)$ y $A_{3} \in W^{q, p-2}\left(\mathbb{R}^{n}, \mathcal{M}\left(N_{3}, N_{4} ; \mathrm{C}\right)\right)$, donde $1 / p_{1}+1 / p_{2}=1$ y $p_{1} \geq 1$, entonces tenemos:

(1) $A_{2} A_{3} \in W^{q, 1}\left(\mathbb{R}^{n}, \mathcal{M}\left(N_{2}, N_{4} ; \mathbb{C}\right)\right)$

(2) $\sup _{y \in \mathbb{R}^{n}}\left\|A_{1}(y)\right\|_{\mathcal{M}}=C_{1}<+\infty \Rightarrow\left\|z^{b} A_{1} A_{2}\right\|_{L^{1}} \leq C_{1}\left\|_{z^{b}} A_{2}\right\|_{L^{1}}$

(3) $\left\|3^{\mathrm{b}} A_{1} *\left[A_{2} A_{3}\right]\right\|_{L^{1}} \leq\left\|3^{\mathrm{b}}\right\| A_{1}\left\|_{\mathcal{M}} *\right\| A_{2} A_{3}\left\|_{\mathcal{M}}\right\|_{L^{1}}$. En particular, si $\sup _{y \in \mathbb{R}^{n}}\left\|A_{2}(y)\right\|_{\mathcal{M}}=C_{2}<+\infty$, entonces

(3') $\left\|z^{\mathrm{b}} A_{1} *\left[A_{2} A_{3}\right]\right\|_{L^{1}} \leq C_{2}\left\|3^{\mathrm{b}}\right\| A_{1}\left\|_{\mathcal{M}} *\right\| A_{3}\left\|_{\mathcal{M}}\right\|_{L^{1}}$.

(4) Si $p_{1}=1$, entonces

$$
\left\|3^{\mathrm{b}}\right\| A_{1}\left\|_{\mathcal{M}} *\right\| A_{2}\left\|_{\mathcal{M}}\right\|_{L^{1}} \leq \sum_{\mathrm{a} \leq \mathrm{b}}\left(\begin{array}{l}
\mathrm{b} \\
\mathrm{a}
\end{array}\right)\left\|3^{\mathrm{a}} A_{1}\right\|_{L^{1}}\left\|z^{\mathrm{b}-\mathrm{a}} A_{2}\right\|_{L^{1}},
$$

donde

$$
\left(\begin{array}{l}
\mathbf{b} \\
\mathbf{a}
\end{array}\right)=\left(\begin{array}{l}
b_{1} \\
a_{1}
\end{array}\right)\left(\begin{array}{l}
b_{2} \\
a_{2}
\end{array}\right) \cdots\left(\begin{array}{l}
b_{n} \\
a_{n}
\end{array}\right) \text { ! }
$$


Demostración. (1) Es consecuencia inmediata de la desigualdad de Hölder y (2), (3) se verifican fácilmente. (3') se sigue directamente de (2) y (3).

(4) Ya que $(y+z)^{\mathbf{b}}=\sum_{\mathbf{a} \leq \mathbf{b}}\left(\begin{array}{l}\mathbf{a} \\ \mathbf{b}\end{array}\right) y^{\mathbf{a} \mathbf{z}^{\mathbf{b}-\mathbf{a}}}$ para $y, z \in \mathbb{R}^{n}$, entonces $\int_{\mathbb{R}^{n}}\left|\mathfrak{z}^{\mathbf{b}}\right| \int_{\mathbb{R}^{n}}\left\|A_{1}(z-y)\right\|_{\mathcal{M}}\left\|A_{2}(y)\right\|_{\mathcal{M}} d y d z=$ $\int_{\mathbb{R}^{n}}\left\|A_{2}(y)\right\|_{\mathcal{M}} \int_{\mathbb{R}^{n}}\left\|z^{\mathbf{b}} A_{1}(z-y)\right\|_{\mathcal{M}} d_{\mathfrak{z}} d y=$ $\int_{\mathbb{R}^{n}}\left\|A_{2}(y)\right\|_{\mathcal{M}} d y \int_{\mathbb{R}^{n}}\left\|(\mathfrak{z}-y)^{\mathbf{b}} A_{1}(\mathfrak{z})\right\|_{\mathcal{M}} d_{\mathfrak{z}} \leq$

$$
\sum_{\mathbf{a} \leq \mathbf{b}}\left(\begin{array}{l}
\mathrm{a} \\
\mathrm{b}
\end{array}\right) \int_{\mathbb{R}^{n}}\left\|y^{\mathrm{a}} A_{2}(y)\right\|_{\mathcal{M}} d y \int_{\mathbb{R}^{n}} \|\left(\mathfrak{z}^{\mathrm{b}-\mathrm{a}} A_{1}(\mathfrak{z}) \|_{\mathcal{M}} d_{\mathfrak{z}}\right.
$$

Como se vió en el capítulo anterior, para cada $f \in C_{0}^{\infty}\left(\mathbb{R}^{n}\right)^{N}$ se tiene $[T(t) f]^{\wedge}(z)=\hat{T}(t)(z) \hat{f}(z)$, donde

$$
\hat{T}(t)(\mathfrak{z}):=\int_{S^{n-1}} e^{n t\langle z, \xi\rangle}(I+\alpha \cdot \xi) d_{s_{n-1}}(\xi) .
$$

Además en el capítulo III también se demostró que

$$
\|\hat{T}(t)\|_{\mathcal{M}} \leq 1 \text {, para } t \in \mathbb{R} .
$$

Ahora, para $s=0,1, \ldots, k, \quad k \in \mathbb{N}$, escribamos $\mathbf{d}=\left(d_{1}, \ldots, d_{k}\right)$ y

$$
o(s, k)=\left\{\mathrm{d} \mid d_{1}+\cdots+d_{k}=s, d_{j} \in\{0,1\} \text { para } j=1, \ldots, k\right\},
$$

entonces para cualesquiera elementos $U, V$ de un anillo $A$ se sabe que

$$
(U+V)^{k}=\sum_{s=0}^{k} \sum_{d \in O(s, k)} \prod_{j=1}^{k}\left[U^{1-d_{j}} V^{d_{j}}\right] .
$$

Tomando ésto en cuenta tenemos 
IV.3 Teorema. Sean $j, \ell \in \mathbb{N}$ y $B \in C_{0}^{\infty}\left(\mathbb{R}^{n}, \mathcal{M}(N, \mathbb{C})\right)$. Para $m \in \mathbb{N}$ y $1 \leq k \leq m$ se cumple:

(a) Hay una constante $M_{1}(j, \ell, B)$ tal que para todo $\phi \in C_{0}^{\infty}\left(\mathbb{R}^{n}\right)^{N}$

$$
\begin{aligned}
\sup _{|t| \leq j}\left\|\left[T(t / m)+\frac{t}{m} B\right]^{k} \phi\right\|_{\ell} & \leq \sup _{|t| \leq j}\left\|\left[\hat{T}(t / m)+\frac{t}{m} \hat{B} *\right]^{k} \hat{\phi}\right\|_{\ell, 1} \\
& \leq M_{1}(j, \ell, B)\|\hat{\phi}\|_{\ell, 1}
\end{aligned}
$$

(b) Si $\phi \in C_{0}^{\infty}\left(\mathbb{R}^{n}\right)^{N}$ y $\phi(x)=0$ para $\|x\| \geq r$, entonces

$$
\sup _{|t| \leq j}\left\|Q(t / m)^{k} \phi\right\|_{\ell} \leq M_{1}^{\prime}(n, j, \ell, B) r^{n} \sup _{\substack{|y| \leq r \\|a| \leq n+\ell+1}}\left\|\partial^{\mathrm{a}} \phi(y)\right\|,
$$

donde $M_{1}^{\prime}(n, j, \ell, B)=M_{1}(j, \ell, B) c(\ell, n) \int_{R^{n}} \frac{d z}{(1+\|z\|)^{n+1}}$

y $c(\ell, n)$ es una constante que no depende de $r$, (ver apéndice A).

Demostración. (a) Para $\mathbf{b}=\left(b_{1}, \ldots, b_{n}\right),|\mathrm{b}|=\ell$, se tiene

$$
\sup _{x \in \mathbb{R}^{n}}\left\|\partial_{x}^{b}\left\{\left[T(t / m)+\frac{t}{m} B\right]^{k} \phi\right\}(x)\right\| \leq\left\|z^{b}\left[\hat{T}(t / m)+\frac{t}{m} \hat{B} *\right]^{k} \hat{\phi}\right\|_{L^{1}} .
$$

De esta desigualdad y la definición de \|\|$_{\ell, 1}$ en (IV.1.1) se obtiene la primer desigualdad. Ahora la fórmula (IV.3.2) da

$$
\mathfrak{z}^{\mathrm{b}}\left[\hat{T}(t / m)+\frac{t}{m} \hat{B} *\right]^{k} \hat{\phi}=\mathfrak{z}^{\mathrm{b}} \sum_{s=0}^{k} \sum_{d \in o(s, k)}\left\{\prod_{j=1}^{k} \hat{T}(t / m)^{1-d_{j}}\left[\frac{t}{m} \hat{B} *\right]^{d_{j}}\right\} \hat{\phi}
$$

Aplicando el lema precedente en cada término de la última suma y tomando en cuenta (IV.3.1),

$$
\begin{aligned}
& \left(\frac{|t|}{m}\right)^{s}\left\|z^{b} \prod_{j=1}^{k} \hat{T}(t / m)^{1-d_{j}}[\hat{B} *]^{d_{j}}\right\|_{L^{1}} \leq \\
& \left(\frac{|t|}{m}\right)^{s}\left\|\mathfrak{z}^{b}\left(\|\hat{B}\|_{\mathcal{M}^{*}}\right)^{d_{1}} \cdots\left(\|\hat{B}\|_{\mathcal{M}^{*}}\right)^{d_{k}} \hat{\phi}\right\|_{L^{1}} \\
& =\left(\frac{|t|}{m}\right)^{s}\left\|z^{b}\left(\|\hat{B}\|_{\mathcal{M}^{*}}\right)^{s} \hat{\phi}\right\|_{L^{1}} \cdot
\end{aligned}
$$


Asi, (4) del lema IV.2 y la relación (IV.3.4) nos llevan a

$$
\begin{aligned}
& \left\|z^{b}\left[\hat{T}(t / m)+\frac{t}{m} \hat{B} *\right]^{k} \hat{\phi}\right\|_{L^{1}} \leq \sum_{s=0}^{k} \sum_{d \in O(s, k)}\left(\frac{|t|}{m}\right)^{s}\left\|z^{b}\left(\|\hat{B}\|_{\mathcal{M}} *\right)^{e}\right\| \hat{\phi}\|\|_{L^{1}}
\end{aligned}
$$

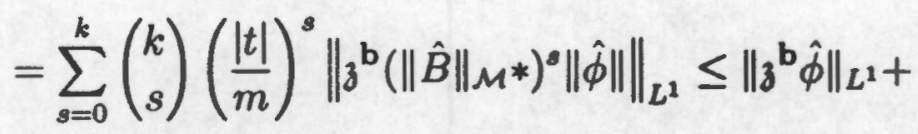

$$
\begin{aligned}
& +\sum_{s=1}^{k}\left(\begin{array}{l}
k \\
s
\end{array}\right)\left(\frac{|t|}{m}\right)^{s} \sum_{b_{s} \leq \cdots \leq b_{0}=b}\left[\prod_{j=0}^{a-1}\left(\begin{array}{c}
b_{j} \\
b_{j+1}
\end{array}\right)\left\|z^{b_{j}-b_{j+1}} \hat{B}\right\|_{L^{1}}\right]\left\|z^{b_{0}} \hat{\phi}\right\|_{L^{1}}
\end{aligned}
$$

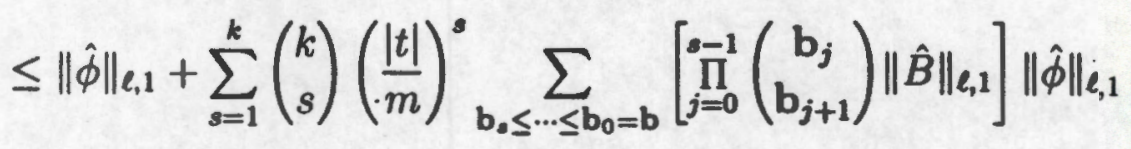

$$
\begin{aligned}
& =\|\hat{\phi}\|_{\ell, 1} \sum_{s=0}^{k}\left(\begin{array}{l}
k \\
s
\end{array}\right)\left(\frac{|t|}{m}\right)^{s}(s+1)^{\ell}\|\hat{B}\|_{\ell, 1}^{s} \text {. }
\end{aligned}
$$

Por otro lado, observemos que para todo $r \geq 0$,

$$
\begin{aligned}
& \sum_{s=0}^{k}\left(\begin{array}{l}
k \\
s
\end{array}\right)\left(\frac{r}{m}\right)^{s}(s+1)^{\ell} \leq 1+\sum_{s=1}^{k}\left(\begin{array}{l}
k \\
s
\end{array}\right)\left(\frac{r}{m}\right)^{s}(2 s)^{\ell} \\
& =1+\left.\left(2 \frac{d}{d \tau}\right)^{\ell}\left(1+\frac{r}{m} e^{\tau}\right)^{s}\right|_{\tau=0} \leq 1+\left.\left(2 \frac{d}{d \tau}\right)^{\ell} e^{r e^{\tau}}\right|_{\tau=0} .
\end{aligned}
$$

De esta manera, (IV.3.5) y (IV.3.6) nos dan

$$
\left\|\mathfrak{z}^{\mathrm{b}}\left[\grave{T}\left(\frac{t}{m}\right)+\frac{t}{m} \hat{B} *\right]^{k} \hat{\phi}\right\|_{L^{1}} \leq\left[1+\left(2 \frac{d}{d \tau}\right)^{\ell} e^{|\ell|\|B\|_{\ell, 1} e^{\tau}}\right]_{\tau=0}\|\hat{\phi}\|_{\ell, 1}
$$

Por lo tanto, para obtener la segunda desigualdad, es suficiente definir

$$
M_{1}(j, \ell, B)=1+\left.\left(2 \frac{d}{d \tau}\right)^{\ell} e^{j\|B\|_{\ell, 1} e^{\tau}}\right|_{\tau=0}
$$

(b) Del apéndice A, (A.3), hay una constante $c(\ell, n)$ independiente de 
$r$ que satisface

$$
\|\hat{\phi}\|_{\ell, 1} \leq c(\ell, n) \int_{\mathbb{R}^{n}} \frac{d z}{(1+\|z\|)^{n+1}} r^{n} \sup _{\substack{|y|<r \\|\mathbf{a}| \leq \ell+n+1}}\left\|\partial^{\mathbf{a}} \phi(y)\right\| .
$$

Escribiendo

$$
M_{1}^{\prime}(n, j, \ell, B)=c(\ell, n) \int_{\mathbb{R}^{n}} \frac{d z}{(1+\|z\|)^{n+1}} r^{n} M_{1}(j, \ell, B)
$$

se obtiene la conclusión deseada.

Ahora prescindimos de la suposición sobre la compacidad de los soportes:

IV.4. Corolario. Sean $\phi \in C^{\infty}\left(\mathbb{R}^{n}\right)$ y $B \in C^{\infty}\left(\mathbb{R}^{n}, \mathcal{M}(N, \mathbb{C})\right)$ arbitrarios. Para cualesquiera $j, \ell \in \mathbb{N}$ podemos encontrar una constante $M_{1}^{n}(n, j, \ell, B)$ tal que para todo $m \geq 2 n j, m \in \mathbb{N}$, se cumple

$$
\sup _{\substack{1 \leq t \leq j \\ 1 \leq k \leq m}}\left\|\left[T(t / m)+\frac{t}{m} B\right]^{k} \phi\right\|_{\ell} \leq M_{1}^{\prime \prime}(n, j, \ell, B)\|\phi\|_{\ell+n+1} .
$$

Demostración. Primero seleccionamos $f_{\ell} \in C_{0}^{\infty}\left(\mathbb{R}^{n}\right)$ con la propiedad

$$
f_{\ell}=1 \text { en }\|y\| \leq \ell+\frac{1}{2} \text { y } f_{\ell}=0 \text { para }\|y\| \geq \ell+1 .
$$

Es inmediato que para toda $\|x\| \leq \ell$,

$$
\begin{aligned}
\{[T(t / m) & \left.\left.+\frac{t}{m} f_{\ell} B\right] f_{\ell} \phi\right\}(x)=f_{\ell}(x)\left\{\left[T(t / m)+\frac{t}{m} B\right] \phi\right\}(x)= \\
& =\left\{\left[T(t / m)+\frac{t}{m} B\right] \phi\right\}(x)=[Q(t / m) \phi](x) .
\end{aligned}
$$

Procediendo de manera semejante obtendremos también para $\|x\| \leq \ell$

$$
\left\{\left[T(t / m)+\frac{t}{m} f_{\ell} B\right]^{k} f_{\ell} \phi\right\}(x)=\left[Q(t / m)^{k} \phi\right](x), \quad k=1,2, \ldots, m .
$$


Ya que $f_{\ell} B$ y $f_{\ell} \phi$ tienen soporte compacto, podemos aplicar (IV.3.3) y (IV.3.7) en (IV.4.1) para conseguir

$$
\sup _{\substack{\| \leq 1 \leq \ell \\|b| \leq \varepsilon^{\ell}, \mid \varepsilon \leq j}}\left\|\left\{\partial^{b}\left[Q(t / m)^{k} \phi\right]\right\}(x)\right\| \leq\left[1+\left(2 \frac{d}{d t}\right)^{\ell} e^{j\left\|\left(s_{\ell} B\right)^{\wedge}\right\| \|_{\ell, 1} e^{\tau}}\right]_{\tau=0}\left\|\left(f_{\ell} \phi\right)^{\wedge}\right\|_{\ell, 1}
$$

Tomando en cuenta (A.6) del apéndice A,

$$
\left\|\left(f_{\ell} \phi\right)^{\wedge}\right\|_{\ell, 1} \leq C(n, \ell) \ell^{n}\left\|f_{\ell}\right\|_{n+\ell+1}\|\phi\|_{n+\ell+1},
$$

entonces deberá cumplirse

$$
\sup _{\substack{\|x\| \leq \ell \\|b| \leq \ell,|t| \leq j}} \|\left\{\partial^{b}\left[Q(t / m)^{k} \phi \mid\right\}(x)\left\|\leq M_{1}^{n}(n, j, \ell, B)\right\| \phi \|_{n+\ell+1},\right.
$$

donde

$$
M_{1}^{\prime \prime}(n, j, \ell, B):=\left[1+\left(2 \frac{d}{d t}\right)^{\ell} e^{j\left\|\left(f_{\ell} B\right)^{\wedge}\right\|_{\ell, 1} e^{\tau}}\right]_{\tau=0}\left\|f_{\ell}\right\|_{n+\ell+1} .
$$

Por definición de \|\|$_{\ell}$, concluímos

$$
\sup _{|t| \leq j}\left\|Q(t / m)^{k} \phi\right\|_{\ell} \leq M_{1}^{n}(n, j, \ell, B)\|\phi\|_{n+\ell+1}, \text { para } k=1,2, \ldots, m .
$$

OBSERVACión. Para cada $\ell \in \mathbb{N}$ el espacio $C^{\infty}(\{|y| \leq \ell\})$ es cerrado en $\mathcal{E}\left(\mathbb{R}^{n}\right)$ y denso en ninguna parte, se sigue que $C_{0}^{\infty}\left(\mathbb{R}^{n}\right)$ es un subespacio de la primera categoría en $\mathcal{E}\left(\mathbb{R}^{n}\right)$. Por esta razón el Teorema de Banach-Steinhaus no puede aplicarse directamente en la demostración del corolario IV.4.

IV.5 Teorema Para $\phi \in \mathcal{E}\left(\mathbb{R}^{n}\right)^{N}$ y $B \in C^{\infty}\left(\mathbb{R}^{n}, \mathcal{M}(N, \mathbb{C})\right)$ arbitrarios hay constantes $M_{i}^{\prime \prime}(n, \ell, B), i=2,3$, tales que para toda $\nu \in \mathbb{N}$
(a) $\sup _{|s| \leq 1 / n}\left\|\frac{d}{d s} Q(s) \phi\right\|_{\ell} \leq 2\left(n^{2}+2^{\ell-1}\|B\|_{l}\right)\|\phi\|_{l+1} ; l^{\prime}=1,2, \ldots$ 
(b) $\sup _{|s| \leq \nu / n}\left\|\frac{d}{d s} Q(s / \nu)^{r} \phi\right\|_{\ell} \leq \frac{r}{\nu} M_{2}^{n}(n, \ell, B)\|\phi\|_{\ell+2 n+3} ; 1 \leq r \leq \nu$

(c) $\sup _{|s| \leq \nu / n}\left\|\left(\frac{d}{d s}\right)^{2} Q(s / \nu)^{\nu} \phi\right\|_{\ell} \leq M_{3}^{\prime \prime}(n, \ell, B)\|\phi\|_{\ell+3 n+5}$

(d) $\sup _{|s| \leq \nu / n} \|\left[Q(s)-Q(s / \nu)^{\nu} \mid \phi\left\|_{\ell} \leq s^{2} M_{3}^{\prime \prime}(n, \ell, B)\right\| \phi \|_{\ell+3 n+5}\right.$, para $1 \leq r \leq \nu$

Demostración. Escribamos $D Q(\tau)=\left(\frac{d}{d \tau} Q\right)(\tau)$ y consideremos $\mathbf{a}=\left(a_{1}, \ldots, a_{n}\right),|\mathbf{a}| \leq \ell$. Para $x \in \mathbb{R}^{n}$ y $\tau \in \mathbb{R}$ con $\|x\| \leq \ell$ y $|\tau| \leq 1 / n$ se tiene:

$$
\begin{aligned}
\left\|\partial^{\mathrm{a}}[D T(\tau) \phi](x)\right\| & =n\left\|\sum_{j=1}^{n} \int_{S^{n-1}}(I+\alpha \cdot \xi) \xi_{j} \frac{\partial}{\partial x_{j}} \partial^{\mathbf{a}} \phi(x+n \tau \xi) d s_{n-1}(\xi)\right\| \\
& \leq n \sum_{j=1}^{n} \int_{S^{n-1}}\|I+\alpha \cdot \xi\|_{\mathcal{M}}\left\|\frac{\partial}{\partial x_{j}} \partial^{\mathbf{a}} \phi(x+n \tau \xi)\right\| d s_{n-1}(\xi) \\
& \leq n \sum_{j=1}^{n} \int_{S^{n-1}} 2\|\phi\|_{\ell+1} d s_{n-1}(\xi)=2 n^{2}\|\phi\|_{\ell+1} .
\end{aligned}
$$

Por otro lado,

$$
\begin{gathered}
\left\|\partial^{\mathbf{a} D} D[\tau B \phi](x)\right\|=\left\|\sum_{\mathbf{b} \leq \mathbf{a}}\left(\begin{array}{l}
a \\
b
\end{array}\right)\left[\partial^{\mathbf{a}-\mathbf{b}} B(x)\right]\left[\partial^{\mathbf{b}} \phi(x)\right]\right\| \\
\leq \sum_{\mathbf{b} \leq \mathbf{a}}\left(\begin{array}{l}
\mathbf{a} \\
\mathbf{b}
\end{array}\right)\|B\|_{\ell}\|\phi\|_{\ell}=2^{|\mathbf{a}|}\|B\|_{\ell}\|\phi\|_{\ell} \leq 2^{\ell}\|B\|_{\ell}\|\phi\|_{\ell+1}
\end{gathered}
$$

Por lo tanto

$$
\|D[T(\tau)+\tau B] \phi\|_{\ell} \leq 2 n^{2}\|\phi\|_{\ell+1}+2^{\ell}\|B\|_{\ell}\|\phi\|_{\ell+1} .
$$

De aquí se sigue (a). Para obtener (b) consideremos la fórmula

$$
\frac{d}{d s} Q(s / \nu)^{r} \phi=\frac{1}{\nu} \sum_{i=1}^{r} Q(s / \nu)^{i-1}[D Q(s / \nu)] Q^{r-i}(s / \nu) \phi .
$$


Aplicando el corolario IV.4 y el inciso anterior llegamos a

$$
\begin{aligned}
\| & \frac{d}{d s} Q(s / \nu)^{r} \phi\left\|_{\ell} \leq \frac{1}{\nu} M_{1}^{\prime \prime}(n, 1, \ell, B) \sum_{i=1}^{r}\right\|[D Q(s / \nu)] Q^{r-i}(s / \nu) \phi \|_{\ell+n+1} \\
& \leq \frac{1}{\nu} M_{1}^{\prime \prime}(n, 1, \ell, B) 2\left(n^{2}+2^{n+\ell}\right) \sum_{i=1}^{r}\left\|Q^{r-i}(s / \nu) \phi\right\|_{\ell+n+2} \\
& \leq \frac{1}{\nu} M_{1}^{\prime \prime}(n, 1, \ell, B) 2\left(n^{2}+2^{n+\ell}\right) M_{1}^{\prime \prime}(n, 1, \ell+n+2, B)\|\phi\|_{\ell+2 n+3} \\
& =\frac{r}{\nu} M_{2}^{\prime \prime}(n, \ell, B)\|\phi\|_{\ell+2 n+3},
\end{aligned}
$$

donde $M_{2}^{\prime \prime}(n, \ell, B)=2\left(n^{2}+2^{n+\ell}\right) M_{1}^{\prime \prime}(n, 1, \ell, B) M_{1}^{\prime \prime}(n, 1, \ell+n+2, B)$.

El mismo argumento combinado con la fórmula

$$
\begin{aligned}
\left(\frac{d}{d s}\right)^{2} Q & (s / \nu)^{\nu} \phi= \\
& =\frac{1}{\nu} \sum_{j=2}^{\nu}\left[\frac{d}{d s} Q(s / \nu)^{j-1}\right][D Q(s / \nu)] Q(s / \nu)^{\nu-j} \phi \\
& +\frac{1}{\nu^{2}} \sum_{j=1}^{\nu} Q(s / \nu)^{j-1}\left[D^{2} Q(s / \nu)\right] Q(s / \nu)^{\nu-j} \phi \\
& +\frac{1}{\nu} \sum_{j=1}^{\nu-1} Q(s / \nu)^{j-1}[D Q(s / \nu)]\left[\frac{d}{d s} Q(s / \nu)^{\nu-j}\right] \phi
\end{aligned}
$$

nos da (c). Resta probar (d). Por la fórmula de Taylor (B.1.1), las relaciones (IV.5.1) y (IV.5.2) valuadas en $s=0$ nos dan

$$
\begin{aligned}
Q(s / \nu)^{\nu} \phi(x) & =\phi(x)+s[(\alpha \cdot \nabla+B) \phi](x)+ \\
& +s^{2} \int_{0}^{1}(1-\theta)\left[D^{2} Q(\theta s / \nu)^{\nu} \phi\right](x) d \theta
\end{aligned}
$$

además

$$
[Q(s) \phi(x)]=\phi(x)+s[(\alpha \cdot \nabla+B) \phi](x)+s^{2} \int_{0}^{1}(1-\theta)\left[D^{2} Q(\theta s) \phi\right](x) d \theta
$$




\section{Entonces}

$$
\left\|Q(s / \nu)^{\nu} \phi-Q(s) \phi\right\|_{l} \leq s^{2} \int_{0}^{1}(1-\theta)\left\|D^{2} Q(\theta s / \nu)^{\nu} \phi-D^{2} Q(\theta s) \phi\right\|_{\ell} d \theta .
$$

Así, las estimaciones anteriores y la desigualdad del triángulo nos conducen a

$$
\begin{aligned}
\left\|Q(s / \nu)^{\nu} \phi-Q(s) \phi\right\|_{\ell} & \leq s^{2} \int_{0}^{1}(1-\theta) 2 M_{3}^{\prime \prime}(n, \ell, B)\|\phi\|_{\ell+3 n+5} d \theta \\
& =s^{2} M_{3}^{n}(n, \ell, B)\|\phi\|_{\ell+3 n+5} .
\end{aligned}
$$

IV.6 Lema. Sea $X$ un espacio de Fréchet cuya topología está definida por una sucesión creciente de seminormas \|\|$_{\ell} \leq\|\|_{\ell+1}, \ell=1,2, \ldots$. Supongamos que $\left\{U_{m}\right\},\left\{V_{m}\right\}$ son sucesiones de operadores en $X$ y $\left\{C_{1}(\ell)\right\},\left\{C_{2}(\ell)\right\}$ son súcesiones en $\mathbb{R}^{+}$tales que para $a, b, d \in \mathbb{N}$ fijos se tiene

$$
\begin{gathered}
\sup _{\substack{m \in \mathbb{N} \\
1 \leq i \leq m}}\left\|U_{m}^{i} u\right\|_{\ell} \leq C_{1}(\ell)\|u\|_{\ell+a ;} \quad \sup _{\substack{m \in \mathbb{N} \\
1 \leq i \leq m}}\left\|V_{m}^{i} u\right\|_{\ell} \leq C_{2}(\ell)\|u\|_{\ell+b} \\
\text { y } \sup _{m \in \mathbb{N}}\left\|U_{m} u-V_{m} u\right\|_{\ell}=\mathcal{O}(\epsilon)\|u\|_{\ell+d}, \text { para } u \in X, \ell \in \mathbb{N} .
\end{gathered}
$$

Entonces para cualesquiera $u \in X$ y $\ell \in \mathbb{N}$ se cumple

$$
\left\|U_{m}^{k} u-V_{m}^{k} u\right\|_{\ell}=k C_{1}(\ell) C_{2}(\ell) \mathcal{O}(\epsilon)\|u\|_{\ell+a+b+d} \text { para } 1 \leq k \leq m .
$$

Demostración. Sean $u \in X$ y $1 \leq k \leq m$, entonces

$$
\left(U_{m}^{k}-V_{m}^{k}\right) u=\sum_{i=0}^{k-1} U_{m}^{i}\left(U_{m}-V_{m}\right) V_{m}^{k-1-i} u
$$

Por lo tinto

$$
\begin{aligned}
\left\|\left(U_{m}^{k}-V_{m}^{k}\right) u\right\|_{\ell} & \leq \sum_{i=0}^{k-1} C_{1}(\ell)\left\|\left(U_{m}-V_{m}\right) V_{m}^{k-1-i} u\right\|_{\ell+\bullet} \\
& \leq \sum_{i=0}^{k-1} C_{1}(\ell) \mathcal{O}(\epsilon)\left\|V_{m}^{k-1-i} u\right\|_{\ell+a+d} \\
& \leq \sum_{i=0}^{k-1} C_{1}(\ell) \mathcal{O}(\epsilon) C_{2}(\ell)\|u\|_{\ell+a+d+b} \\
& =k C_{1}(\ell) C_{2}(\ell) \mathcal{O}(\epsilon)\|u\|_{\ell+a+d+b}
\end{aligned}
$$


IV.7 Proposición. Dados $j, \ell \in \mathbb{N}$ fijos y $1 \leq k \leq m, m \in \mathbb{N}$, tenemos (a) $\sup _{|t| \leq j}\left\|\left[Q(t / k !)^{k !}-Q(t / m !)^{m !}\right] \phi\right\|_{\ell}=\mathcal{O}(j / k !)\|\phi\|_{\ell+5 n+6}, \quad \phi \in \mathcal{E}\left(\mathbb{R}^{n}\right)^{N}$ En particular $Q(t / m !)^{m !}$ es una sucesión de Cauchy en $\mathcal{B}\left(\mathcal{E}\left(\mathbb{R}^{n}\right)^{N}\right)$. (b) Si escribimos $e(t) \phi=\lim _{m} Q(t / m !)^{m !} \phi, \phi \in \mathcal{E}\left(\mathbb{R}^{n}\right)^{N}$, entonces $e \in C^{1}\left(\mathbb{R}, \mathcal{B}\left(\mathcal{E}\left(\mathbb{R}^{n}\right)^{N}\right), \sup _{|t| \leq j}\|e(t) \phi\|_{\ell} \leq M^{n}(j, n, \ell, B)\|\phi\|_{\ell+n+1}\right.$ y $e(s+t)=e(s) e(t)$ para $s, t \geq 0$.

Demostración. Para $1 \leq k \leq m$ definamos $U_{k l}=Q(t / k !)$ y $V_{k l}=$ $Q(t / m !)^{m ! / k !}$. Por el corolario IV.4,

$\left\|U_{k !} \phi\right\|_{\ell} \leq M_{1}^{\prime \prime}(j, n, \ell, B)\|\phi\|_{\ell+n+1} ;\left\|V_{k !} \phi\right\|_{\ell} \leq M_{1}^{\prime \prime}(j, \dot{n}, \ell, B)\|\phi\|_{\ell+n+1}$.

Ahora hacemos $s=t / k !, \nu=m ! / k ! \in \mathbb{N}$ y aplicamos (d) del Teorema IV.5 para obtener

$$
\|\left[U_{k !}-V_{k !} \mid \phi\left\|_{\ell}=\mathcal{O}\left(s^{2}\right)\right\| \phi\left\|_{\ell+3 n+5}=\mathcal{O}\left((t / k !)^{2}\right)\right\| \phi \|_{\ell+3 n+5} .\right.
$$

De aquí, el lema anterior nos dá

$$
\begin{aligned}
\sup _{|t| \leq j} \|\left[Q(t / k !)^{k !}-Q(t / m !)^{m !} \mid \phi \|_{\ell}\right. & =k ! \mathcal{O}\left((j / k !)^{2}\right)\|\phi\|_{\ell+3 n+5+2(n+1)} \\
& =\mathcal{O}\left(j^{2} / k !\right)\|\phi\|_{\ell+5 n+6} .
\end{aligned}
$$

De esta relación vemos que para todo $t \in \mathbb{R}$, el operador

$$
e(t):=\lim _{m} Q(t / m !)^{m !} \in \mathcal{B}\left(\mathcal{E}\left(\mathbb{R}^{n}\right)^{N}\right)
$$

está bien definido y (a) queda mostrado.

En virtud del teorema IV.5-(c), para cada $m \geq n j$ obtenemos

$$
\sup _{|t| \leq j}\left\|\left(\frac{d}{d t}\right)^{2} Q(t / m !)^{m !} \phi\right\|_{\ell} \leq M_{3}^{*}(n, \ell, B)\|\phi\|_{\ell+3 n+5}
$$


Así, la parte (a) anterior y el teorema (B.2) implican la diferenciabilidad continua de $e$. La estimación para $e(t)$ es consecuencia inmediata de su definición. Finalmente, el razonamiento empleado en (a) con $s=$ $k t / m !, \nu=k$ y IV.5-(d) nos sirven para verificar que dado $k \in \mathbb{N}$ fijo tendremos

$$
\left\|Q(k t / m !)^{m !} \phi-Q(t / m !)^{m ! k} \phi\right\|_{\ell}=\mathcal{O}\left(t^{2} / m !\right)\|\phi\|_{\ell+5 n+6},
$$

en consecuencia para $k \in \mathbb{N}$ arbitrario

$$
e(k t)-e(t)^{k} \bar{\beta}_{\bar{\beta}} \lim _{m \rightarrow \infty}\left[Q(k t / m !)^{m !}-Q(t / m !)^{m ! k}\right]=0 .
$$

Se sigue que para cualesquiera racionales positivos, $\frac{p_{1}}{q_{1}}, \frac{p_{2}}{q_{2}}$;

$$
e\left(\frac{p_{1}}{q_{1}}+\frac{p_{2}}{q_{2}}\right)=e\left(\frac{1}{q_{1} q_{2}}\right)^{p_{1} q_{2}} e\left(\frac{1}{q_{1} q_{2}}\right)^{p_{2} q_{1}}=e\left(\frac{p_{1}}{q_{1}}\right) e\left(\frac{p_{2}}{q_{2}}\right) .
$$

Por la continuidad de $e$ la conclusión es inmediata.

Hemos llegado al resultado principal de este capítulo.

IV.8 Teorema. Para $B \in C^{\infty}\left(\mathbb{R}^{n}, \mathcal{M}(N, \mathbb{C})\right)$ escribamos

$$
\begin{gathered}
Q(t / m)^{m}=\left[T(t / m)+\left.(t / m) B\right|^{m}, \quad m=1,2,3, \ldots\right. \\
\text { y } \quad e(t)=\lim _{\boldsymbol{m} \rightarrow \infty} Q(t / m !)^{m !}
\end{gathered}
$$

entonces

(a) En el espacio $C^{\infty}\left(\mathbb{R}, \mathcal{B}\left(\mathcal{E}\left(\mathbb{R}^{n}\right)^{N}\right)\right)$, e es la única solución de la ecuación diferencial

$$
\frac{d \psi}{d t}=(\alpha \cdot \nabla+B) \psi, \quad t \in \mathbb{R}, \quad \psi(0)=I
$$

(b) Para cualesquiera $t_{1}, t_{2} \in \mathbb{R}, e\left(t_{1}+t_{2}\right)=e\left(t_{1}\right) e\left(t_{2}\right)=e\left(t_{2}\right) e\left(t_{1}\right)$

(c) Los siguientes límites

$$
e(t)=\lim _{m} Q(t / m)^{m},
$$




$$
\frac{d e(t)}{d t}=\lim _{m} \frac{d}{d t} Q(t / m)^{m}
$$

existen uniformemente en $t$ para cualquier intervalo compacto de $\mathbb{R}$.

Demostración. Por la proposición IV.7 vemos que $e(0)=I, e^{\prime}(0)=$ $(\alpha \cdot \nabla+B)$ y $e(s+t)=e(s) e(t)$ para $s, t \geq 0$. Así,

$$
\begin{aligned}
\frac{d e(t)}{d t} & =\left.\frac{d}{d s}\right|_{s=0^{+}} e(s+t)=\left.\frac{d}{d s}\right|_{s=0^{+}} e(s) e(t)=e^{\prime}(0) e(t) \\
& =(\alpha \cdot \nabla+B) e(t)=\left.\frac{d}{d s}\right|_{\omega=0^{+}} e(t) e(s)=e(t)(\alpha \cdot \nabla+B), \quad t \geq 0 .
\end{aligned}
$$

Ya que $-\alpha=\left(-\alpha_{1}, \ldots,-\alpha_{n}\right)$ y $-B$ tienen las mismas propiedades de $\alpha$ y $B$, podemos definir los operadores correspondientes:

$$
\begin{aligned}
& {\left[T_{-}(t) \phi\right](x)=\int_{s_{n-1}}(I-\alpha \cdot \xi) \phi(x+n t \xi) d s_{n-1}(\xi), t \in \mathbb{R}} \\
& Q_{-}(t)=T_{-}(t)-t B, t \in \mathbb{R} \\
& e_{-}(t)_{\bar{\beta}}-\lim _{m} Q_{-}(t / m !)^{m !}, t \in \mathbb{R} .
\end{aligned}
$$

El razonamiento previo implica

$$
\frac{d e_{-}(t)}{d t}=-(\alpha \cdot \nabla+B) e_{-}(t)=-e_{-}(t)(\alpha \cdot \nabla+B), t \geq 0
$$

Por lo tanto $\frac{d}{d t}\left[e_{-}(t) e(t)\right]=0, t \geq 0$.

En consecuencia

$$
e_{-}(t) e(t)=e_{-}(0) e(0)=I, \quad t \geq 0
$$

Es claro que $Q_{-}(t)=Q(-t)$ luego, (IV.8.2) nos lleva a $e_{-}(t)=e(-t)$ para $t \in \mathbb{R}$. En virtud de (IV.8.6) obtenemos

$$
e(-t)=e(t)^{-1}, \quad t \geq 0 .
$$

Por métodos elementales se concluye (b). 
Para demostrar (a), observemos que la parte (b) nos permite extender (IV.8.1) para todo $t \in \mathbb{R}$, i.e.,

$$
\frac{d e(t)}{d t}=(\alpha \cdot \nabla+B) e(t)=e(t)(\alpha \cdot \nabla+B), \quad t \in \mathbb{R} .
$$

Ahora, el operador diferencial $(\alpha \cdot \nabla+B)$ no incluye derivadas respecto al tiempo $t$ y por ésto la suavidad es evidente. Si $g$ es una solución de (IV.8.3), entonces $\frac{d}{d t}\left(g e^{-1}\right)=0$ y se concluye que $g(t)=e(t)$ para $t \in \mathbb{R}$.

Para finalizar con (c), fijemos $j \in \mathbb{N}$. Si $\ell \in \mathbb{N}$ es arbitrario, el corolario IV.4, el teorema IV.5 y la proposición IV.7 implican

$$
\begin{gathered}
\sup _{|t| \leq j}\left\|Q(t / m)^{k} \phi\right\|_{\ell} \leq M_{1}^{\prime \prime}(j, \ell, n, B)\|\phi\|_{\ell+n+1,} \\
\sup _{|t| \leq j}\|e(t / m) \phi\|_{\ell} \leq M_{1}^{n}(j, \ell, n, B)\|\phi\|_{\ell+n+1}, \quad \text { y } \\
\sup _{|t| \leq 1 / n}\left\|D^{2} Q(s) \phi\right\|_{\ell} \leq M_{3}^{n}(n, \ell, B)\|\phi\|_{\ell+3 n+5} .
\end{gathered}
$$

Por otro lado, la fórmula de Taylor nos dá

$[Q(s)-e(s)] \phi(x)=s^{2} \int_{0}^{1}(1-\theta)\left[D^{2} Q(\theta s)-e(\theta s)(\alpha \cdot \nabla+B)^{2}\right] \phi(x) d \theta$.

De este modo y tomando en cuenta que

$$
\begin{aligned}
\sup _{|\ell| \leq j}\left\|e(\tau)(\alpha \cdot \nabla+B)^{2} \phi\right\|_{\ell} & \leq M_{1}^{\prime \prime}(j, \ell, n, B)\left\|(\alpha \cdot \nabla+B)^{2} \phi\right\|_{\ell+n+1} \\
& \leq M_{1}^{\prime \prime}(j, \ell, n, B)\left(1+2^{2 \ell+n+2}\right)\|\phi\|_{\ell+n+3}
\end{aligned}
$$

entonces, para $m \geq n j$ obtenemos

$$
\begin{aligned}
\sup _{|t| \leq j} \|\left[Q(t / m)-e(t / m) \mid \phi \|_{\ell}\right. & \leq \text { const. }(j, \ell, n, B)\|\phi\|_{\ell+3 n+5}(t / m)^{2} \\
& =\mathcal{O}\left(t^{2} / m^{2}\right)\|\phi\|_{\ell+3 n+5} .
\end{aligned}
$$

Aplicando el lema IV. 6 a los operadores

$$
U_{m}=Q(t / m), \quad V_{m}=e(t / m) ; \quad|t| \leq j, \quad m \in \mathbb{N},
$$


se llega a

$$
\begin{aligned}
\sup _{|t| \leq j}\left\|\left[Q(t / m)_{*}^{m}-e(t)\right] \phi\right\|_{\ell} & =\left\|\left[U_{m}^{m}-V_{m}^{m}\right] \phi\right\|_{\ell}= \\
& =\mathcal{O}\left(j^{2} / m\right)\|\phi\|_{\ell+5 n+7} \underset{m \rightarrow \infty}{\longrightarrow} 0 .
\end{aligned}
$$

Ahora resta probar el segundo límite: Para cada $m \in \mathbb{N}$,

$$
\begin{gathered}
\frac{d}{d t} Q(t / m)^{m}-\frac{d}{d t} e(t)=\frac{d}{d t} Q(t / m)^{m}-e(t / m)^{m}(\alpha \cdot \nabla+B) \\
=\sum_{k=1}^{m}\left[Q(t / m)^{k-1}[D Q](t / m) Q(t / m)^{m-k}-e(t / m)^{k-1}(\alpha \cdot \nabla+B) e(t / m)^{m-k}\right] \\
=\sum_{k=1}^{m}\left[U_{m}^{k-1}[D Q](t / m) U_{m}^{m-k}-V_{m}^{k-1}(\alpha \cdot \nabla+B) V_{m}^{m-k}\right] .
\end{gathered}
$$

Cada término de esta suma es igual a

$$
\begin{gathered}
{\left[U_{m}^{k-1}-V_{m}^{k-1}\right] D Q(t / m) U_{m}^{m-k}+V_{m}^{k-1}[(D Q)(t / m)-(\alpha \cdot \nabla+B)] U_{m}^{m-k}} \\
+V_{m}^{k-1}(\alpha \cdot \nabla+B)\left[U_{m}^{m-k}-V_{m}^{m-k}\right] .
\end{gathered}
$$

Por las relaciones (IV.8.7) y (IV.8.8),

$$
\left\|\left[U_{m}-V_{m}\right] \phi\right\|_{\ell}=\mathcal{O}\left(t^{2} / m^{2}\right)\|\phi\|_{\ell+3 n+5},
$$

entonces el lema asegura que

$$
\left\|\left[U_{m}^{r}-V_{m}^{r}\right] \phi\right\|_{\ell}=\mathcal{O}\left(t^{2} / m^{2}\right)\|\phi\|_{\ell+5 n+7}, \text { para } r=1,2, \ldots, m .
$$

Por otro lado, el polinomio de Taylor implica

$$
\|[D Q(t / m)-(\alpha \cdot \nabla+B)] \phi\|_{\ell}=\mathcal{O}(t / m)\|\phi\|_{\ell+5 n+7},
$$

por lo linto, para alguna constante adecuada $a(n)$ independiente de $\ell$, se deberí tener

$$
\begin{aligned}
\sup _{|\ell| \leq j}\left\|\left[\frac{d}{d t} Q\left(\frac{t}{m}\right)^{m}-\frac{d}{d t} e(t)\right] \phi\right\|_{\ell}=\frac{1}{m} \sum_{k=1}^{m}\left[(k-1) \mathcal{O}\left(j^{2} / m^{2}\right)\|\phi\|_{\ell+a(n)}\right. \\
\left.+\mathcal{O}(j / m)\|\phi\|_{\ell+a(n)}+(m-k) \mathcal{O}\left(j^{2} / m^{2}\right)\|\phi\|_{\ell+a(n)}\right]= \\
=\mathcal{O}\left(\frac{j+j^{2}}{m}\right)\|\phi\|_{\ell^{+} a(n)} \underset{m \rightarrow \infty}{\longrightarrow} 0 .
\end{aligned}
$$




\section{APENDICE A}

1. Si $K$ es un subconjunto compacto de $\mathbb{R}^{n}$, escribimos

$$
C^{\infty}(K)^{N}=\left\{\phi \in C^{\infty}\left(\mathbb{R}^{n}\right)^{N} \mid \text { soporte }(\phi) \subset K\right\} .
$$

Dado $r>0$, sea

$$
B_{r}=\left\{y \in \mathbb{R}^{n} \mid\|y\| \leq r\right\} .
$$

Si $\phi \in C^{\infty}\left(B_{r}\right)^{N}$, entonces para cada multíndice a se tiene

$$
(i z)^{a} \hat{\phi}(z)=(2 \pi)^{-n / 2} \int_{\mathbb{R}^{n}}\left(\partial^{a} \phi\right)(y) e^{-i(z, y)} d y, \quad z \in \mathbb{R}^{n} .
$$

Por lo tanto, para cualesquiera $|a| \leq m$ y $z \in \mathbb{R}^{n}$,

$$
\left|z^{a}\right|\|\hat{\phi}(z)\| \leq \operatorname{Vol}\left(B_{r}\right) \sup _{|y| \leq r}\left\|\partial^{a} \phi(y)\right\| \leq \operatorname{Vol}\left(B_{1}\right) r^{n} \sup _{\substack{\| n|\leq r\\| b \mid \leq m}}\left\|\partial^{b} \phi(y)\right\| .
$$

Se sigue que para todo $z \in \mathbb{R}^{n}$,

$$
\begin{aligned}
& (1+\|\mathfrak{z}\|)^{m}\|\hat{\phi}(\mathfrak{z})\| \leq \\
& \sum_{k=0}^{m}\left(\begin{array}{c}
m \\
k
\end{array}\right) \sum_{|\mathbf{a}|=k}\left(\begin{array}{c}
k \\
a_{1}
\end{array}\right)\left(\begin{array}{c}
k-a_{1} \\
a_{2}
\end{array}\right) \cdots\left(\begin{array}{c}
k-\left(a_{1}+\cdots+a_{n-1}\right) \\
a_{n}
\end{array}\right)\left|z^{a}\right|\|\hat{\phi}(\mathfrak{z})\| \\
& \leq(1+n)^{m} \operatorname{Vol}\left(B_{1}\right) r^{n} \sup _{\substack{|y| \leq r \\
|\mathbf{b}| \leq m}}\left\|\partial^{\mathbf{b}} \phi(y)\right\|
\end{aligned}
$$

Así,

$$
\|\hat{\phi}(z)\| \leq \frac{c_{1}(n, m)}{(1+\|z\|)^{m}} r^{n} \sup _{\substack{n, y \| \leq r \\|b| \leq m}}\left\|\partial^{b} \phi(y)\right\|, \quad z \in \mathbb{R}^{n},
$$

donde $c_{1}(n, m)=(1+n)^{m} \operatorname{Vol}\left(B_{1}\right)$. En particular

$$
\|\hat{\phi}(3)\| \leq \frac{c_{1}(n, m)}{\left(1+\left\|_{3}\right\|\right)^{m}} r^{n}\|\phi\|_{m} \quad \text { para } \phi \in C^{\infty}\left(B_{r}\right)^{N} \text {. y } m \geq r
$$


2. Como consecuencia inmediata de (A.1) obtenemos

$$
\begin{aligned}
\|\hat{\phi}\|_{m, 1} & :=\int_{\mathbb{R}^{n}}\left(1+{ }^{\prime}\|\mathfrak{z}\|\right)^{m}\|\hat{\phi}(\mathfrak{z})\| d \mathfrak{z} \\
& \leq\left(c_{1}(n, m+n+1) \int_{\mathbb{R}^{n}} \frac{(1+\|z\|)^{m} d_{\mathfrak{z}}}{(1+\|z\|)^{m+n+1}}\right) r^{n} \sup _{\substack{\| v|\leq r\\
| b_{1} \leq m+n+1}}\left\|\partial^{b} \phi(y)\right\|,
\end{aligned}
$$

i.e.,

$$
\|\hat{\phi}\|_{m, 1} \leq c_{2}(n, m) r^{n} \sup _{\substack{\| v|\leq r\\| \mathbf{b} \mid \leq m+n+1}}\left\|\partial^{\mathbf{b}} \phi(y)\right\|_{;} \quad \phi \in C^{\infty}\left(B_{r}\right)^{N},
$$

donde $c_{2}(n, m)=c_{1}(n, m+n+1) \int_{\mathbb{R}^{n}} \frac{(1+\|z\|)^{m} d z}{(1+\|z\|)^{m+n+1}}$.

En particular

$$
\|\hat{\phi}\|_{m, 1} \leq c_{2}(n, m) r^{n}\|\phi\|_{m+n+1} \text { para } \phi \in C^{\infty}\left(B_{r}\right)^{N} \text { y } m \geq \dot{r} .
$$

3. Sean $A_{1} \in C^{\infty}\left(\mathbb{R}^{n}, \mathcal{M}\left(m_{1}, m_{2} ; \mathbb{C}\right)\right)$ y $A_{2} \in C^{\infty}\left(\mathbb{R}^{n}, \mathcal{M}\left(m_{2}, m_{3} ; \mathbb{C}\right)\right)$. Si a es un multiíndice, la fórmula de Leibniz nos da

$$
\partial^{\mathrm{a}}\left(A_{1} A_{2}\right)=\sum_{\mathbf{b} \leq \mathbf{a}}\left(\begin{array}{l}
a_{1} \\
b_{1}
\end{array}\right) \cdots\left(\begin{array}{l}
a_{n} \\
b_{n}
\end{array}\right)\left(\partial^{b} A_{1}\right)\left(\partial^{a-b} A_{2}\right) .
$$

De aquí,

$$
\left\|A_{1} A_{2}\right\|_{m} \leq 2^{m}\left\|A_{1}\right\|_{m}\left\|A_{2}\right\|_{m} .
$$

Combinando (A.4) y (A.5) obtenemos

$$
\left\|(f A)^{\wedge}\right\|_{m, 1} \leq c_{2}(n, m) 2^{m+n+1} r^{n}\|f\|_{m+n+1}\|A\|_{m+n+1},
$$

donde $f \in C^{\infty}\left(B_{r}\right)$ y $A \in C^{\infty}\left(\mathbb{R}^{n}, \mathcal{M}\left(n_{1}, n_{2} ; \mathbb{C}\right)\right)$.

\section{APENDICE B}


1. Sea $X$ un espacio de Fréchet con seminormas \|\|$_{1} \leq\|\|_{2} \leq \cdots \leq$ \|\|$_{k} \leq\|\|_{k+1} \leq \cdots$. Una familia. $\mathcal{A} \subset X$ es acotada si y solo si

$$
M_{\ell}=\sup _{u \in \mathcal{A}}\|u\|_{\ell}<\infty \text { para } \ell=1,2, \ldots
$$

La seminorma correspondiente de un operador acotado $T: X \rightarrow X$ es dada por

$$
\|T\|_{\ell, \mathcal{A}}:=\sup _{u \in \mathcal{A}}\|T u\|_{\ell}, \quad \mathcal{A} \subset X \text { acotado. }
$$

Como se acostumbra, $\mathcal{B}(X)$ denota el espacio de operadores acotados en $X$ dotado cơn la topología generada por la familia de seminormas \| \|es. En esta topología, la convergencia de una sucesión $\left\{U_{m}\right\} \subset \mathcal{B}(X)$ se denotará

$$
U=\lim _{m} U_{m} \text {. }
$$

En particular la derivada de una familia uniparamétrica $\{L(t)\}$ está definida por

$$
D L(t)=\lim _{\lambda \rightarrow 0} \frac{L(t+\lambda)-L(t)}{\lambda} \in \mathcal{B}(X)
$$

Suponiendo que $U \in C^{k}([-\epsilon, \epsilon], \mathcal{B}(X))$, la fórmula de Taylor asegura que

$$
\begin{aligned}
U(t) u & =\sum_{j=0}^{k-1}\left(t-t_{0}\right)^{j} \frac{D^{j} U\left(t_{0}\right)}{j !} u+ \\
& +\frac{\left(t-t_{0}\right)^{k}}{(k-1) !} \int_{0}^{1}(1-\theta)^{k-1} D^{k} U\left(t_{0}+\theta\left(t-t_{0}\right)\right) u d \theta, u \in X
\end{aligned}
$$

De ello se sigue que si $V \in C^{k}([-\epsilon, \epsilon], \mathcal{B}(X))$ y $a, b \in \mathbb{N}$ son fijos y satisfacen

$$
\begin{aligned}
D^{j} U(0)=D^{j} V(0) & , \text { para } 1 \leq j \leq(k-1) \text { y si además } \\
& \sup _{|s| \leq \epsilon}\left\|D^{k} U(s) u\right\|_{\ell} \leq M_{1}\|u\|_{\ell+a} \\
& \sup _{|s| \leq \epsilon}\left\|D^{k} V(s) u\right\|_{\ell} \leq M_{2}\|u\|_{\ell+b}
\end{aligned}
$$


entonces para cada $i=1, \ldots,(k-1)$ y cada $u \in X$,

$$
\sup _{|\tau| \leq \epsilon}\left\|D^{i}[U-V](\tau) u\right\|_{\ell}=\mathcal{O}\left(\left(\tau-t_{0}\right)^{k-i}\right)\|u\|_{\ell+c}, \quad \ell=1,2, \ldots,
$$

donde $c=\max \{a, b\}$.

Decimos que $X$ tiene la propiedad de Heine-Borel si para cada sucesión acotada $\left\{U_{m}\right\} \subset X$ podemos encontrar una subsucesión convergente $\left\{U_{m_{n}}\right\}_{n}$, i.e., hay un único $u \in X$ tal que

$$
\lim _{n}\left\|U_{m_{n}}-u\right\|_{\ell}=0, \quad \text { para } \ell=1,2, \ldots
$$

Como ejemplos de espacios de Fréchet con la propiedad de Heine-Borel tenemos $\mathbb{R}^{N}, \mathcal{E}\left(\mathbb{R}^{n}\right)^{N}$, el espacio de Shwartz $\mathcal{S}\left(\mathbb{R}^{n}\right)^{N}$ y

$$
W_{p}\left(\mathbb{R}^{n}\right)=\left\{f \in \mathbb{C}^{\infty}\left(\mathbb{R}^{n}\right): D^{k} f \in L^{p}\left(\mathbb{R}^{n}\right), k=0,1, \ldots\right\} .
$$

2. Teorema. Sean $X$ un espacio de Fréchet con la propiedad de HeineBorel y $V \in C^{0}([-\epsilon, \epsilon], \mathcal{B}(X))$. Supongamos que existen una sucesión $\left\{U_{m}\right\}_{m=1}^{+\infty} \subset C^{2}([-\epsilon, \epsilon], \mathcal{B}(X))$ y funciones $f_{\ell}, g_{\ell}: X \rightarrow \mathbb{R}^{+}, \ell=1,2, \ldots$ tales que:
(a) $\lim _{m}\left[\sup _{|\tau| \leq \varepsilon}\left\|U_{m}(\tau) u-V(\tau) u\right\|_{\ell}\right]=0, \quad \ell \in \mathbb{N}$ y $u \in X$
(b) $\sup _{|\tau| \leq \epsilon}\left\|D^{2} U_{m}(\tau) u\right\|_{\ell} \leq f_{\ell}(u) ; \quad \ell \in \mathbb{N}$ y $u \in X$
(c) Existe $\left|t_{0}\right|<\epsilon$ con la propiedad

$$
\left\|D U_{m}\left(t_{0}\right) u\right\|_{\ell} \leq g_{\ell}(u) ; \ell \in \mathbb{N} \text { y } u \in X .
$$

Entonces $V \in C^{1}((-\epsilon, \epsilon), \mathcal{B}(X))$.

Demostración. Fijemos $u \in X$. Aplicando la condición (b) y la desigualdad del Valor Medio obtenemos

$$
\left\|D U_{m}(\tau) u-D U_{m}(s) u\right\|_{\ell} \leq|\tau-s| f_{\ell}(u) ; \quad s, \tau_{!} \in[-\epsilon, \epsilon] .
$$


Se sigue que $\left\{D U_{m}(\cdot) u\right\} \subset C^{0}([-\epsilon, \epsilon], X)$ es una familia equicontinua. Tomando $s=t_{0}$ en la desigualdad anterior, la hipótesis (b) y la desigualdad del triángulo nos dan

$$
\left\|D U_{m}(\tau) u\right\|_{\ell} \leq g_{\ell}(u)+\left|\tau-t_{0}\right| f_{\ell}(u), \quad|\tau|<\epsilon .
$$

Por lo tanto, para cada $|\tau|<\epsilon$, el conjunto $\mathcal{H}=\left\{D U_{m}(\tau) u\right\}_{m}$ es acotado en $X$. Puesto que $X$ tiene la propiedad de Heine-Borel, la $X$ cerradura de $\mathcal{H}$ es compacto. Por el Teorema de Arzelá-Ascoli existen una subsucesión $\left\{D U_{m}(\cdot) u\right\}$ y $h_{u} \in C^{0}([-\epsilon, \epsilon], X)$ tales que

$$
\lim _{k \rightarrow+\infty}\left[\sup _{|\xi| \leq \epsilon}\left\|D U_{m_{k}}(\xi) u-h_{u}(\xi)\right\|_{\ell}\right]=0 ; \quad \ell=1,2,3, \ldots
$$

Para cada $s, \tau \in[-\epsilon, \epsilon]$ se tiene:

$$
\begin{aligned}
& V(s) u-V(\tau) u-(s-\tau) h_{u}(\tau)=\left[V(s) u-U_{m_{k}}(s) u\right]+ \\
& +\left[U_{m_{k}}(\tau) u-V(\tau) u\right]+\left[U_{m_{k}}(s) u-U_{m_{k}}(\tau) u-(s-\tau) h_{u}(\tau)\right] .
\end{aligned}
$$

Por la fórmula de Taylor,

$$
\begin{aligned}
U_{m_{k}}(s) u-U_{m_{k}}(\tau) u= & (s-\tau) D U_{m_{k}}(\tau) u \\
& +(s-\tau)^{2} \int_{0}^{1}(1-\theta) D^{2} U_{m_{k}}(s+\theta(\tau-s)) u d \theta
\end{aligned}
$$

De esta manera, (B.6), (B.7) y (b) implican

$$
\begin{aligned}
& \left\|V(s) u-V(\tau) u-(s-\tau) h_{u}(\tau)\right\|_{\ell} \leq 2 \sup _{|\xi| \leq \varepsilon}\left\|U_{m_{k}}(\xi) u-V(\xi) u\right\|_{\ell} \\
& +|s-\tau| \sup _{|\xi| \leq \epsilon}\left\|D U_{m_{k}}(\xi) u-h_{u}(\xi)\right\|_{\ell}+\frac{|s-\tau|^{2}}{2} f_{\ell}(u) .
\end{aligned}
$$

Tomando lim en ambos miembros de esta desigualdad, la hipótesis (a) y la relación (B.5) nos conducen a

$$
\left\|V(s) u-V(\tau) u-(s-\tau) h_{u}(\tau)\right\|_{l} \leq \frac{|s-\tau|^{2}}{2} h_{l}(u)
$$


y obtenemos

$$
\left\|\frac{V(s) u-V(\tau) u}{s-\tau}-h_{u}(\tau)\right\|_{\ell} \leq \frac{|s-\tau|}{2} f_{\ell}(u) ; \quad s, \tau \in[-\epsilon, \epsilon] .
$$

Así, para cada $u \in X$ existe $h_{u} \in C^{0}([-\epsilon, \epsilon], X)$ tal que

$$
h_{u}(\tau) \lim _{\bar{X} \rightarrow \tau} \frac{[V(s)-V(\tau)]}{s-\tau} u ; \quad|\tau|<\epsilon
$$

De esta manera podemos contruir la aplicación

$$
H:(-\epsilon, \epsilon) \times X \rightarrow X, \quad H(\tau, u)=h_{u}(\tau) .
$$

Si $|\tau|<\epsilon$ es fijo, el Teorema de Banach-Steinhaus aplicado a la familia de operadores $\left\{\Lambda_{s, \tau}=\frac{V(s)-V(\tau)}{s-\tau}: s \neq \tau\right\}$ junto con (B.10) nos permiten mostrar que $H(\tau, \cdot) \in \mathcal{B}(X)$. De este razonamiento se sigue también que hay constantes $C_{\ell}>0$ y $a(\ell)>0$ tales que

$$
\sup _{|s|<\varepsilon}\left\|\Lambda_{\mathbf{s}, \tau} u-H(\tau, u)\right\|_{\ell} \leq C_{\ell}\|u\|_{a(\ell)}
$$

Para ver que $V \in C^{1}((-\epsilon, \epsilon), \mathcal{B}(X))$ debemos mostrar

$$
\left.\begin{array}{l}
H(\tau, \cdot)=\lim _{\beta \rightarrow \tau} \frac{V(s)-V(\tau)}{s-\tau}=\lim _{s \rightarrow \tau} \Lambda_{s, \tau} \\
H(\tau, \cdot)=\lim _{s \rightarrow \tau} H(s, \cdot) .
\end{array}\right\}
$$

En efecto, sean $\mathcal{A} \subset X$ acotado y $\delta>0$. Si $\overline{\mathcal{A}}$ denota la $X$-cerradura de $\mathcal{A}$, entonces $\overline{\mathcal{A}}$ es un espacio métrico compacto y, por lo tanto, para cada $\ell \in \mathbb{N}$ hay un número finito de puntos $u_{1}, u_{2}, \ldots, u_{m_{\ell}} \in \overline{\mathcal{A}}$ con la propiedad

$$
\min _{1 \leq j \leq m_{\ell}}\left\|\omega-u_{j}\right\|_{\ell}<\frac{\delta}{2\left(1+C_{\ell}\right)}, \quad \omega \in \bar{A} .
$$

De este modo, para cada $\omega \in \overline{\mathcal{A}}$ escogemos un $u_{j}$ adecuado y aplicamos las relaciones (B.9) y (B.11) para obtener

$$
\left\|\Lambda_{\ell, \tau} \omega-H(\tau, \omega)\right\|_{\ell} \leq\left\|\Lambda_{\ell, \tau}\left(\omega-u_{j}\right)-H\left(\tau, \omega-u_{j}\right)\right\|_{\ell}+\left\|\dot{\Lambda}_{s, \tau} u_{j}-H\left(\tau, u_{j}\right)\right\|_{\ell}
$$




$$
\leq C_{\ell}\left\|\omega-u_{j}\right\|_{a(\ell)}+\frac{|s-\tau|}{2} f_{\ell}\left(u_{j}\right)<\frac{\delta}{2}+\frac{|s-\tau|}{2} f_{\ell}\left(u_{j}\right) .
$$

Escogiendo $M=\max \left\{f_{\ell}\left(u_{j}\right) ; \quad 1 \leq j \leq m_{\ell}\right\}$ y $|s-\tau|<\frac{\delta}{1+M}$, tomando en cuenta que $\Lambda_{\mathrm{s}, \tau}=\Lambda_{\tau, \boldsymbol{s}}$ para $s \neq \tau$, la desigualdad anterior implica

$$
\sup _{\omega \in \mathcal{A}}\left\|\Lambda_{s, \tau} \omega-H(\tau, \omega)\right\|_{\ell} \leq \frac{\delta}{2}+\frac{|s-\tau|}{2} M<\delta
$$

y

$$
\begin{aligned}
\sup _{\omega \in \mathcal{A}}\|H(s, \omega)-H(\tau, \omega)\|_{\ell} & \leq \sup _{\omega \in \mathcal{A}}\left[\left\|H(s, \omega)-\Lambda_{\tau, \boldsymbol{A}} \omega\right\|_{\ell}+\left\|\Lambda_{\boldsymbol{s}, \tau} \omega-H(\tau, \omega)\right\|_{\ell}\right] \\
& \leq \delta+|s-\tau| M<2 \delta .
\end{aligned}
$$

Esto demuestra (B.12) y concluye la prueba del teorema. 


\section{Referencias}

1. a) Berenstein, C., Taylor, B.A.; "A New look at interpolation theory for entire functions of one variable", Advances in Mathematics 33 (1979), 109-143. b) Berenstein, C., Gay, Yger.

c) Berestein, C., Zalcman.

2. Brown, L., Schreiber B.M., Taylor B.A.; "Spectral Synthesis and the Pompeiu problem", Ann. Inst. Fourier (3) 1973.

3. Corona Gulmaro.

4. a) Chargoy Jesús

b)

c)

d)

5. Ehrenpreis L.; "Fourier Analysis in Several Complex Variables", WileyInterscience, New York, 1970.

6. a) Folland Gerald B; "Introduction to Partial Differential Equations". Mathematical Notes, Princeton University Press, 1976.

b) Folland Gerald, Stein E.M.: "Hardy Spaces on Homogeneous Groups", Math. Notes, Princeton Univ. Press, 1982.

7. Gaveau B, Schulman.

8. Gelfand-Shilov; "Generalized Functiond I-IIP", Academic Press, 1964.

9. Gurevich.; "Counterexamples to a problem of L. Schwartz", Functional Anal. Appl. 9 (1975) pp. 116-120.

10. a) Helgason Sigudur "The Randon Transform", Birkhäuser, 1980.

b) Helgason Sigudur "Topics in Harmonic Analysis on Homogeneous Spaces", 1981. 
11. Hewitt E., Ross K.; "Abstract Harmonic Analysis", Springer Verlag, 1970.

12. Hörmander Lars; "The Analysis of Partial Diff. Operators, I-IV" Springer Verlag, 1983.

13. John Fritz; "Partial Diff. Equations", Springer-Verlag, 1982.

14. Kostantinov.

15. Levin B.J.; "Zeros of Entrie Functions", Translations of Math. Monographs, Vol.V, AMS, 1964.

16. Mikhailov V.P.; "Partial Diff. Equations", MIR, 1982.

17. Naimark M.A.; "Linear Diff. Operators I, IP", Ungar, 1968.

18. Nelson.

19. Quezada.

20. a) Rudin Walter; "Real and Complex Analysis", Mc Graw-Hill.

b) Rudin Walter; "Functional Analysis", Mc Graw-Hill.

21. a) Scwartz L.; "Théorie générale des functions moyenns periodics", Ann of Math. (2) 48, 1947.

b) Schwartz L.; "Théorie des Distributions", Hermann, 1960.

22. Warner (i; "Harmonic Analysis on Semisimple Lie Groups", SpringerVerlag, 1972.

23. a) Wawrzyńczyk Antoni "Special Functions and Group Representation Theory", P.W.N. Warzawa, 1984.

b) Wawrzyńczyk Antoni "Spectral Analysis and Synthesis on Symmetric Spaces", Journal of Math. Analysis and Applications.

c)

24. Yosida-Kôsaku; "Functional Analysis", Springer-Verlag, 1978. 


\section{. КАДЕМИЯ НАУК СОIОЗА ССР \\ РЕДАКЦИЯ ЖУРНАЛА «MATEMATИЧЕСКИЕ ЗАMETКИ» ИЗДАНИЕ ГЛАВНОЯ РЕДАКЦИИ ФИЗИКО-МАТЕМАТИЧЕСКОИ ЛИТЕРАТУРЫ ИЗДАТЕЛЬСТВА СНАУКА*}

117071, Москва В-71, Ленинскй проспект, д. 15.

Телефон 234-08-26 No

Ha Ne

$\Gamma$

December 26, 1993, MOSCOW

Dear Professors J.CHARGOY and R.QUEZADA,

We are pleased to inform you that your paper "Solutions of the Weyl and Dirac equations by means of spheric averages" is accepted for publication by Editorial Board of "Mathematical Notices". After translation in Russian the paper will appear in the nearest volume of the magazine. English translation will be published by Plenum Press Company (London) in 1994.

Scincerely yours,

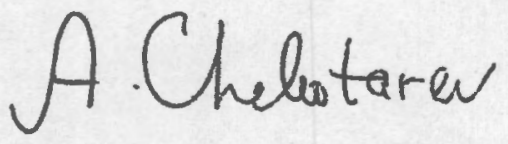
Alexander Chebotarev Executive Secretary 


\section{Sometido a Mathematical Notes, Mosan, 1993.}

\section{APPROXIMATIONS TO THE SOLUTION OF THE WEYL AND THE DIRAC EQUATIONS BY MEANS OF SPHERICAL AVERAGES.}

\section{J. Chargoy \& R: Qứezada ${ }^{1}$.}

1. According to a representation formula given in [5] and [2], the solution of the Dirac equation can be written as the expectation of a random variable, which is defined in terms of the unitary group associated with the Weyl equation. Our main aim in the present work is to express the unitary group associated with the Weyl equation, as the limit of suitable defined spherical averages.

For any smooth enough initial function $\phi(x), x \in \mathbb{R}^{n}$, we define the spherical average

$$
[T(t) \phi](x)=\int_{S^{n-1}} d \mu(v) \phi(x+n t v)
$$

where $\mu$ is the complex matrix-valued measure on $S^{n-1}$ defined by $d \mu(v)=$ $(I+\alpha \cdot v) d s_{n-1}(u)$, with $\alpha \cdot v=\sum_{j=1}^{n} \alpha_{j} v_{j}, \alpha_{1}, \ldots, \alpha_{n}$ being $N \times N$-complex matrices (the Dirac matrices), $\left(v=v_{1}, \ldots, v_{n}\right) \in S^{n-1}$ and $d s_{n-1}(v)$ is the normalized Lebesgue measure on $S^{n-1}$. We shall prove that the solution of the Weyl equation with initial condition $\phi(x)$, is the limit in $\left[L^{2}\left(\mathbb{R}^{n}\right)\right]^{N}$ of the sequence $[T(t / k)]^{k} \phi(x)$. Combining this approximations for the solution of the Weyl equation with the probabilistic formula given in [5], we obtain that the solution of the Dirac equation can be written as the expectation of the limit of spherical averages. The representations obtained in this manner can be considered as path integrals for the Weyl and the Dirac equations, i.e., the solution of these equations is written as an integral over an infinitedimensional space of trajectories in $S^{n-1}$, defined by means of the limit of integrals over finite-dimensional spaces which could be interpreted as spaces of poligonal trajectories.

A similar scheme of approximation was considered firstly in [1], where spherical averages similar to ours were used. The speed of the involved trajectories, which equals $n$ is our approach, is not important to estimate the

\footnotetext{
${ }^{1}$ Departamento de Matemáticas, Universidad Autónoma Metropolitana-Iatapalapa, Av, Michoacán y la Purísima, Col. Vicentina, 09340, Iztapalapa D.F. MEXICO.
} 
norm of the operator $T(t)$, but to get that $\left.\frac{d T}{d t}\right|_{t=0}$ equals the Weyl hamiltonian.

The anticommuting variables involve in the Dirac equation can be represented in severat different manners. In this paper we use mainly the more traditional representation of these variables by means of complex matrices, it is more appropriate to estimate a priori the norm of our operator $T(t)$. Nevertheless in the last section, we describe a scalar representation of $T(t)$, which is based on a representation of the Dirac matrices as scalar operators which act as superposition of shifts of arguments and phases [3].

2. The Dirac equation is a partial differential equation of the form

$$
-i \partial_{\ell} \psi(t, x)=\left[\sum_{j=1}^{n} \alpha_{j}\left(-i \partial_{x_{j}}+e A_{j}(x)\right)+e V+m \beta\right] \psi(t, x)
$$

where $\psi$ is a wave function belonging to a suitable Hilbert space $\mathbb{H I}, A_{j}$ $(j=1,2, \ldots, n)$ and $V$ are real-valued functions (the potentials); $\alpha_{j}(j=$ $1,2, \ldots, n)$ and $\alpha_{n+1}=\beta$ are anticommuting variables satisfying

$$
\alpha_{j} \alpha_{k}+\alpha_{k} \alpha_{j}=\delta_{j k}, \quad \text { for } j, k=1,2, \ldots, n \text {. }
$$

The equation is written in units so that $c=\hbar=1, m$ is the rest mass and $e$ is the charge.

The anticommuting variables $\alpha_{j}$ can be represented by means of complex matrices of a certain size $N \times N$, and the situable Hilbert space $\mathbb{H}$ can be choosen as the space $\left[L^{2}\left(\mathbb{R}^{n}\right)\right]^{N}=\left\{\phi=\left(\phi_{1}, \phi_{2}, \ldots, \phi_{N}\right): \phi_{j} \in L^{2}\left(\mathbb{R}^{n}\right)_{2}, \leq\right.$ $j \leq N\}$, provided with the inner product

$$
(\phi, \psi)=\sum_{k=1}^{N} \int_{\mathbb{R}^{n}} \phi_{k}(x) \bar{\psi}_{k}(x) d x
$$

For the sake of simplicity we will consider in this paper only the free Dirac equation, i.e., we shall assume that $V(x)=0$ and $A_{j}(x)=0$ for $x \in \mathbb{R}^{n}$ and $j=1,2, \ldots, n$. This restriction is not essential, non-zero potentials can be included, for example, by defining spherical averages which depend on them. 
In [5] it was proved that the solution of the free Dirac equation can be written in the form

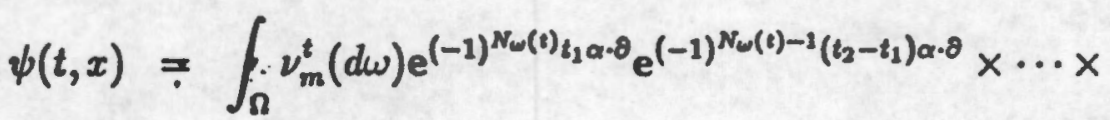

$$
\begin{aligned}
& x \mathrm{e}^{\left(t-t_{N_{0}}\right) a \cdot \theta} \phi(x) \text {. }
\end{aligned}
$$

where $\alpha \cdot \partial=\sum_{j=1}^{n} \alpha_{j} \partial_{s_{j}},(\Omega, \mathbb{F}, P)$ is the probability space associated with a counting Poisson process $N_{\omega}(t)$ with intensity $m$ (the rest mass), $0<t_{1}<$ $\cdots<t_{N_{\omega}}<t$ are the jump times of this process and $\nu_{n}^{t}$ is the $N \times N$-complex matrix valued measure on $\Omega$ so that $d \nu_{m}^{t}=e^{m t}(i \beta)^{N_{\omega}(t)} d P$.

Each one of the factors involved in this representation equals the unitary group associated with the Weyl equation

$$
\partial_{t} \eta(t, x)=(\alpha \cdot \partial) \eta(t, x)
$$

3. We shall prove in this section that the solution of the Weyl equation with initial condition $\phi \in\left[C_{0}^{\infty}\left(\mathbb{R}^{n}\right)\right]^{N}$ can be approximated in $\left[L^{2}\left(\mathbb{R}^{n}\right)\right]^{N}$ by the sequence $[T(t / k)]^{k} \phi(x)$, with $T(t)$ the operator defined by (1.1). By taking derivative with respect to $t$ in (1.1) we have

$$
\begin{aligned}
\frac{d}{d t}[T(t) \phi](x) & =\int_{s^{n-1}}(I+\alpha \cdot v) \frac{d}{d t}(\phi(x+n t v)) d s_{n-1}(v) \\
& =n \int_{S^{n-1}}(I+\alpha \cdot v) v \cdot \partial \phi(x+n t v) d s_{n-1}(v)
\end{aligned}
$$

$\phi \in\left[C_{0}^{\infty}\left(\mathbb{R}^{n}\right)\right]^{N}$. Now it is easy to see that

$$
\int_{S^{n-1}} v_{j} d s_{n-1}(v)=0 \text { and } \int_{S^{n-1}} v_{j} v_{k} d s_{n-1}(v)=\frac{\delta_{j k}}{n}, \text { for } j, k=1,2, \ldots, n \text {. }
$$


So in $t=0$ we obtain $T(0)=I$ and

$$
\begin{aligned}
\frac{d}{d t}[T(t) \phi](x) & =n \sum_{j=1}^{n}\left(\int_{S^{n-1}} v_{j} d s_{n-1}(v)\right) \partial_{x_{j}} \phi(x) \\
& +n \sum_{j \neq k}\left(\int_{S^{n-1}} v_{j} v_{k} d s_{n-1}(v)\right) \alpha_{j} \partial_{x_{k}} \phi(x) \\
& +n \sum_{j=1}^{n}\left(\int_{S^{n-1}} v_{j}^{2} d s_{n-1}(v)\right) \alpha_{j} \partial_{x_{j}} \phi(x) \\
& =\alpha \cdot \partial \phi(x), \quad \phi \in\left[C_{0}^{\infty}\left(\mathbb{R}^{n}\right)\right]^{N}
\end{aligned}
$$

The same is true for any $\phi \in \mathcal{D}(\alpha \cdot \partial)=\left[W_{2,1}\left(\mathbb{R}^{n}\right)\right]^{N}$, where $W_{2,1}\left(\mathbb{R}^{n}\right)$ is the Sobolev space of order 1 . So we can conclude that

$$
\left.\frac{d T}{d t}\right|_{t=0}=\alpha \cdot \partial \text { on } \mathcal{D}(\alpha \cdot \partial) \text {. }
$$

In order to prove that the sequence $[T(t / k)]^{k} \phi(x)$ converges to the solution of the Weyl equation we need the following

Lemma 3.1. For any $\tau>0$, the operator $T(\tau)$ defined by (1.1) is a contraction, i.e., $\|T(\tau)\|_{\mathcal{B}(\mathbb{H I})} \leq 1$.

We will prove this assertion in section 5 .

Theorem 3.2. For each $\phi \in\left[C_{0}^{\infty}\left(\mathbb{R}^{n}\right)\right]^{N}$ we have that

$$
\lim _{k \rightarrow \infty}[T(t / k)]^{k} \phi(x)=\mathrm{e}^{t \alpha \cdot \theta} \phi(x) .
$$

Proof. We will follow the proof given by Nelson for the Trotter product formula (see [4]).

Given $\phi \in \mathcal{D}(\alpha \cdot \partial)=\left[W_{2,1}\left(\mathbb{R}^{n}\right)\right]^{N}$ we have

$$
\begin{aligned}
\lim _{t \rightarrow 0} \frac{1}{t}\left(T(t)-e^{t \alpha \cdot \theta}\right) \phi & =\lim _{t \rightarrow 0}\left[\frac{1}{t}(T(t) \phi-\phi)-\frac{1}{t}\left(e^{t \alpha \cdot \theta} \phi-\phi\right)\right] \\
& =\alpha \cdot \partial \phi-\alpha \cdot \partial \phi=0 .
\end{aligned}
$$


It follows that $\left\{\frac{1}{t}\left(T(t)-\mathrm{e}^{t a \cdot \theta}\right) \phi: t>0\right\}$ is a bounded subset of $\mathbb{H}$. The principle of uniform boundedness applied to the Banach spaces $\mathbb{H}$ and $D(\alpha \cdot \partial)$ ensures the existence of a constant $c$ such that

$$
\sup _{t>0}\left\|\frac{1}{t}\left(T(t)-\mathrm{e}^{t \alpha \cdot \theta}\right) \phi\right\|_{\mathbb{H}} \leq C\|\phi\|_{2,1}, \quad \phi \in \mathcal{D}(\alpha \cdot \partial)
$$

where \|\|$_{2,1}$ denotes the norm in $\left[W_{2,1}\left(\mathbb{R}^{n}\right)\right]^{N}$.

That is to say, the family of operators

$$
\frac{1}{t}\left(T(t)-e^{t \alpha \cdot \theta}\right): \mathcal{D}(\alpha \cdot \partial) \rightarrow \mathbb{H}, \quad t>0
$$

is a uniformly bounded set of operators which tends strongly to zero when $t \rightarrow 0$. A simple argument permits us to conclude that $\left\|\frac{1}{t}\left(T(t)-e^{t_{\alpha} \cdot \theta}\right) \phi\right\|_{\mathbb{H}} \rightarrow$ 0 , as $t \rightarrow 0$, uniformly on compact subsets of $D(\alpha \cdot \partial)$. By observing that, for every $t>0$ and $\phi \in\left[C_{0}^{\infty}\left(\mathbb{R}^{n}\right)\right]^{N}$, the map $:[0, t] \rightarrow \mathcal{D}(\alpha \cdot \partial), s \mapsto \mathrm{e}^{s \alpha \cdot \theta}$, is continuous, we see that the set $\left\{\mathrm{e}^{o \alpha \cdot \theta}: 0 \leq s \leq t\right\}$ is a compact subset of $\mathcal{D}(\alpha \cdot \partial)$. Therefore given $\epsilon>0$, there is $k_{0} \in \mathbb{N}$ such that

$$
\sup _{\substack{0<s \leq t \\ k \geq k_{0}}}\left\|\frac{t}{k}\left[T(t / k)-\mathrm{e}^{\frac{t}{k} \alpha \cdot \theta}\right] \mathrm{e}^{s \alpha \cdot \theta} \phi\right\|_{\mathbb{H}}<\epsilon .
$$

On the other hand we have

$$
[T(t / k)]^{k}-\mathrm{e}^{t \alpha \cdot \theta}=\sum_{j=0}^{k-1}[T(t / k)]^{j}\left(T(t / k)-\mathrm{e}^{\frac{k}{k \alpha \cdot \theta}}\right) \mathrm{e}^{\frac{k-j^{j}}{k} t_{\alpha} \cdot \theta} .
$$

Because of Lemma 3.1 and the relations (3.6), (3.7), we obtain the following estimate:

$$
\begin{aligned}
\left\|\left([T(t / k)]^{k}-e^{t \alpha \cdot \theta}\right) \phi\right\|_{\mathbb{H}} \leq \sum_{j=0}^{k=1}\left\|\left(T(t / k)-\mathrm{e}^{\frac{t}{k} \alpha \cdot \theta}\right) \mathrm{e}^{\frac{k-i^{k}}{k} \alpha \cdot \theta} \phi\right\|_{\mathbb{H}} \\
\leq \sum_{j=0}^{k-1} \frac{t}{k}\left\|\frac{1}{(t / k)}\left(T(t / k)-\mathrm{e}^{t a \cdot \theta}\right) \mathrm{e}^{\frac{(k-j)}{k} t \alpha \cdot \theta} \phi\right\|_{\mathbb{H}} \\
<\sum_{j=0}^{k-1} \frac{t}{k} \epsilon=t \epsilon, \quad k \geq k_{0}
\end{aligned}
$$


so the theorem holds.

4. Now we shall giye the corresponding approximations for the solution of the Dirac equation. For $k \in \mathbb{N}$ and $\phi \in\left[C_{0}^{\infty}\left(\mathbb{R}^{n}\right)\right]^{N}$ let us define the functions

$$
\begin{array}{ll}
\phi_{k}(t, \dot{x}, \omega) & =\left[T\left(t_{1} / k\right)\right]^{k}\left[T\left(\frac{t_{2}-t_{1}}{k}\right)\right]^{k} \cdots\left[T\left(\frac{t-t_{N_{\omega_{k}}}}{k}\right)\right]^{k} \phi(x) \quad \text { and } \\
\psi_{k}(t, x) & =\int_{\Omega} \nu_{m}^{t}(d \omega) \phi_{k}(t, x, \omega)
\end{array}
$$

where $\omega \in \Omega$ and $0<t_{1}<t_{2}<\cdots<t_{N_{\omega}}<t$ are the jump times of the process $N_{\omega}(t)$.

By repeating the arguments given in the proof of Thm. 3.2, we can see that for each $\omega \in \Omega$.

$$
\begin{aligned}
\lim _{k} \phi_{k}(t, x, \omega) & =\lim _{k}\left(\left[T\left(t_{1} / k\right)\right]^{k} \cdots\left[T\left(\frac{t-t_{N_{\omega}}}{k}\right)\right]^{k} \phi(x)\right) \\
& =\mathrm{e}^{(-1)^{N_{\omega}(t) t_{1} \alpha \cdot \partial} \cdots \mathrm{e}^{\left(t-t_{N_{\omega}}\right) \alpha \cdot \theta} \phi(x) .}
\end{aligned}
$$

By virtue of Lemma 3.1 we get

$$
\left\|\left[T\left(t_{1} / k\right)\right]^{k} \cdots\left[T\left(\frac{t-t_{N_{\omega}}}{k}\right)\right]^{k}\right\|_{\mathcal{B}(\mathbb{H})} \leq 1, \quad k \in \mathbb{N} .
$$

Since

$$
\left|\int_{\Omega} \nu_{m}^{t}(d \omega)\right| \leq \int_{\Omega} \mathrm{e}^{m t}\left|(i \beta)^{N_{\omega}(t)}\right| P(d \omega)=\mathrm{e}^{m t},
$$

then the function $g(\omega)=1$ is $\nu_{m}^{t}$-integrable. By applying Dominated Convergence Theorem, the representation (2.3) and Fubini's Theorem, it follows that the sequence $\psi_{k}(t, x)$ converges in $\left[L^{2}\left(\mathbb{R}^{n}\right)\right]^{N}$ to the solution of the Dirac equation with initial condition $\phi(x)$.

\section{Proof of Lemma 3.1}

Because of the Plancherel Theorem, the Fourier transform, $\wedge$, is an isometry and we will employ this result to calculate the norm of the operator $T(\tau)$ for $\tau>0$. We have

$$
\left((T(\tau) \phi)^{\wedge}(p)=\left[\int_{S^{n-1}}(I+\dot{\alpha} \cdot v) e^{i n \tau p-v} d s_{n-1}(v)\right] \hat{\phi}(P) .\right.
$$


By defining

$$
\begin{aligned}
& I_{n}(n \tau p)=I_{N \times N} \int_{S^{n-1}} e^{i n \tau p \cdot v} d s_{n-1}(v), \text { and } \\
& \vdots \vdots \\
& Q_{n}(\tau, p)=\int_{S^{n-1}}(I+\alpha \cdot v) e^{i n \tau p \cdot v} d s_{n-1}(v),
\end{aligned}
$$

we can see that

$$
Q_{n}(\tau, p)=I_{n}(n \tau p)+\frac{1}{i n \tau} \alpha \cdot \nabla_{p} I_{n}(n \tau p) \in \mathcal{B}\left(C^{n}\right), \tau>0
$$

It is easy to check that $I_{n}$ is invariant under rotations, so we can write $I_{n}(n \tau p)=g_{n}(n \tau|p|) I_{N \times N}$ where $g_{n}$ is an even smooth function of one real variable. Furthermore

$$
\Delta_{p} I_{n}(n \tau p)=-n^{2} \tau^{2} I_{n}(n \tau p), \quad p \in \mathbb{R}^{n} .
$$

It follows that $g_{n}$ satisfies the ordinary differential equation,

$$
g_{n}^{\prime \prime}+\frac{(n-1)}{r} g_{n}^{\prime}+g_{n}=0, \quad r>0, \quad g_{n}(0)=1
$$

Here, by definition, | | denotes the usual euclidean norm on $\mathbf{C}^{n}$, and $g_{n}^{\prime}=$ $d / d r\left(g_{n}\right)$.

Thus we get

$$
Q_{n}(\tau, p)=g_{n}(n \tau|p|) I_{N \times N}+\frac{\alpha \cdot p}{i|p|} g_{n}^{\prime}(n \tau|p|)
$$

A straightforward computation shows that for every $u \in \mathbb{C}^{n},|u|=1$, we have

$$
\left|Q_{n}(\tau, p) u\right|^{2}=g_{n}^{2}(n \tau|p|)+\left(g_{n}^{\prime}\right)^{2}(n \tau|p|) \text {. }
$$

Now let us define $g(s)=g_{n}^{2}(s)+\left(g_{n}^{\prime}\right)^{2}(s), s \geq 0$. Because of the relation (5.5) we obtain

$$
g^{\prime}(s)=2 g_{n}^{\prime}(s)\left[g_{n}(s)+g_{n}^{\prime \prime}(s)\right]=-\frac{2(n-1)}{s}\left[g_{n}^{\prime}(s)\right]^{2} \leq 0, \quad s>0, \quad n \geq 2,
$$


i.e., $g$ is a monotone non-increasing function. Hence $g(s) \leq g(0)=1, s \geq 0$. Consequently

$$
\sup _{p \in \mathbb{R}^{n}, \uparrow>0}\left\|Q_{n}(\tau, p)\right\|_{\mathcal{B}\left(\mathbb{C}^{n}\right)}^{2}=\sup _{\substack{|| \mid=1 \\ p \in \mathbb{R}^{n}, \rightarrow>0}}\left|Q_{n}(\tau, p) u\right|^{2} \leq g(0)=1 .
$$

In particular

$$
\| T(\tau) \phi]\left.^{\wedge}(p)\right|^{2}=\left|Q_{n}(\tau, p) \hat{\phi}(p)\right|^{2} \leq|\hat{\phi}(p)|^{2} \quad p \in \mathbb{R}^{n}, \quad \phi \in \mathbb{H} .
$$

After an integration we conclude that for any $\tau>0$,

$$
\|T(\tau) \phi\|_{\mathbb{H}}=\left\|[T(\tau) \phi]^{\wedge}\right\|_{\mathbb{H}} \leq\|\hat{\phi}\|_{\mathbb{H}}=\|\phi\|_{\mathbb{H}}, \quad \phi \in \mathbb{H},
$$

and the proof is complete.

6. To close this paper we describe a scalar representation of $T(t)$ by introducing shifts of arguments and phases. We shall consider the abelian group $\mathbb{Z}_{2} \times \mathbb{Z}_{2}$ and the bijective map $\xi:\{1,2,3,4\} \longrightarrow \mathbb{Z}_{2} \times \mathbb{Z}_{2}$ given by $\xi(1)=(0,0)$, $\xi(2)=(0,1), \xi(3)=(1,0), \xi(4)=(1,1)$.

We denote by $\mathcal{H}$ the orthogonal direct sum $\oplus_{1}^{4} L^{2}\left(\mathbb{R}^{3}\right)$ and by $G$ the abelian group $\mathbb{R}^{3} \times \mathbb{Z}_{2} \times \mathbb{Z}_{2}$. The Haar invariant measure $\mu$ on $G$ is given by

$$
\int_{G} \varphi d \mu=\frac{1}{4} \sum_{j=1}^{4} \int_{\mathbb{R}^{3}} \varphi(x, \xi(j)) d x .
$$

For $\psi=\left(\psi_{1}, \psi_{2}, \psi_{3}, \psi_{4}\right) \in \mathcal{H}$, the formula

$$
U \psi(x, \xi(j))=\psi_{j}(x), \quad j=1,2,3,4
$$

induces a unitary isomorphism $U: \mathcal{H} \rightarrow L^{2}(G)$. Write $S=\left\{0, \frac{1}{2},-\frac{1}{2}, 1\right\}$ and let $\mathcal{S}$ denote the interval $[0,1)$ with the sum module 1 , i.e.,

$$
x_{1} \dot{+} x_{2}=\left\{\begin{array}{ll}
x_{1}+x_{2} & \text { if } 0 \leq x_{1}+x_{2}<1 \\
1-x_{1}-x_{2} & \text { if } x_{1}+x_{2} \geq 1
\end{array} .\right.
$$

For $g=\left(x, z_{1}, z_{2}\right) \in G$, we shall consider the maps $a, b: G \times \mathbb{Z}_{2} \times \mathbb{Z}_{2} \rightarrow \mathbb{Z}_{2}$, $s: G \times \mathbb{Z}_{2} \times \mathbb{Z}_{2} \rightarrow \mathcal{S}$ given in the following table: 


$:$\begin{tabular}{|c|c|c|c|c|}
\hline$\xi$ & $(0,0)$ & $(0,1)$ & $(1,0)$ & $(1,1)$ \\
\hline$a(g, \xi)$ & 1 & 1 & 1 & 0 \\
\hline$b(g, \xi)$ & 1 & 1 & 0 & 0 \\
\hline$s(g, \xi)$ & 0 & $z_{1}-1 / 2$ & $z_{2}$ & $z_{1}$ \\
\hline
\end{tabular}

and we define

$$
\begin{array}{lll}
f: G \times \mathbb{Z}_{2} \times \mathbb{Z}_{2} \rightarrow G \times \mathbb{Z}_{2} \times \mathbb{Z}_{2} & , f(g, \xi)=(0, a(g, \xi)) \\
F: G \times \mathbb{Z}_{2} \times \mathbb{Z}_{2} \rightarrow G \times \mathcal{S}, & F(g, \xi)=(f(g, \xi), s(g, \xi))
\end{array}
$$

The equivalent representation of the Dirac matrices $\left\{\alpha_{j}\right\}_{j=1}^{4}$ is given by

$$
\hat{\alpha}_{j}=U \alpha_{j} U^{*} \in \mathcal{B}\left(L^{2}(G)\right), \quad j=1,2,3,4,
$$

(see [3]), which, acts as a superposition of shifts of arguments and phases:

$$
\left(\hat{\alpha}_{j} \varphi \mathrm{e}^{i \pi \sigma}\right)(g)=\varphi\left(g \dot{+} f(g, \xi(j)) \mathrm{e}^{i \pi(\sigma \dot{+} s(g, \xi(j)))},\right.
$$

where $\dot{+}$ denotes the corresponding group operation in $G$ or $S$. By considering the canonical lifting

$$
\Im: L^{2}(G) \rightarrow L^{2}(G \times S) ;(\Im \varphi)(g, \sigma)=\varphi(g) \mathrm{e}^{i \pi \sigma}
$$

and the action of the Dirac matrices as a restriction to the submanifold $\{(g, 0): g \in G\} \subset G \times S$ of shifts in $G \times S$, we get:

$$
\left(\hat{\alpha}_{j} \varphi\right)(g)=\Im \varphi((g, 0) \dot{+} F(g, \xi(j))) .
$$

For every $(g, \sigma) \in G \times \mathcal{S}$ we define the corresponding shift

$$
D_{(g, \sigma)}: L^{2}(G \times \mathcal{S}) \rightarrow L^{2}(G \times \mathcal{S}), \quad D_{(g, \sigma)}: \phi(q)=\phi(q \dot{+}(g, \sigma)) .
$$

Given $\omega=\left(\omega_{1}, \omega_{2}, \omega_{3}\right) \in \mathbb{R}^{3}$ we write

$$
Q(\omega)=(3 \omega, 0,0,0) \in G \times \mathcal{S} \text { and } \omega(\xi)=\left(\omega_{1}, \dot{\omega}_{2}, \omega_{3}, 0\right) .
$$

Therefore

$$
\begin{aligned}
& U \psi(x+3 t v)=D_{Q(t v)} U \psi(x), \quad \text { and } \\
& v_{j}\left(\hat{\alpha}_{j} U \psi\right)(g)=v_{j} D_{F(g, \xi(j))} \Im U \psi(g, 0) .
\end{aligned}
$$


By writing $\phi=\Im U \psi$ we obtain the scalar representation of $T(t)$,

$$
\begin{aligned}
(U T(t) \psi)(g) & =\int_{S^{2} \times \mathbb{Z}_{2} \times \mathbb{Z}_{2}} D_{Q(t v)}\left[I+v(\xi) \cdot D_{F(g, \xi)] \phi(g, 0) d \mu(v, \xi)}\right. \\
& =\frac{1}{4} \sum_{j=1}^{1} \int_{S^{2}} D_{Q(t v)}\left[I+4 v(\xi(j)) \cdot D_{F(g, \xi)}\right] \phi(g, 0) d s_{n-1}(v) .
\end{aligned}
$$

Let us denote the direct product of a finite number of the normed integrals $\int_{S^{2} \times \mathbb{Z}_{2} \times \mathbb{Z}_{2}}$ as a mathematical expectation $E$, then

$$
[U T(t) \psi](g)=E D_{Q(t v)}\left(I+4 v(\xi) \cdot D_{F(g, \xi)}\right) \phi(g, 0)
$$

and

$$
\left[U T(t / N)^{N} \psi\right](g)=E D_{Q\left(\frac{t}{N} \sum_{k=1}^{N} v_{k}\right)} \prod_{k=1}^{N}\left(I+4 v\left(\xi_{k}\right) \cdot D_{F\left(g_{k-1}, \xi_{k}\right)}\right) \phi(g, 0),
$$

where $g_{0}=g \in G$ and $g_{k}=g_{k-1} \dot{+} F\left(g_{k-1}, \xi_{k}\right)$.

Acknowledgments: We are deeply grateful with Alexander Chebotarev for fruitful discussions during the preparation of this paper. 


\section{Literature Cited.}

1. Corona, G.; "A one parameter family of approximations of the solution of the initial Cauchy problem for the Dirac equation", J. Phys. A: Math. Gen. '22, 2341, (1989).

2. B. Gaveau and L.S. Schulman; "Grassmann-valued processes for the Weyl and the Dirac equations", Phys. Rev. D, $\underline{36}, 1135,(1987)$.

3. Kontantinov, A.A.; Maslov, V.P.; Chebotarev, A.M.; "Probabilistic representation of solutions of the Cauchy problem for the Schrödinger, Pauli and Dirac equations", Russian Math. Surveys 45.6, 1-26, (1988).

4. Nelson E.; "Feynman integrals and the Schrödinger equation, of J. Math. Phys. $\underline{5}, 332$, (1964).

5. Quezada, R.; "Path integral for Dirac equation in momentum space", Univ. Iagellonicae, Acta Math. Vol. 29, (1990). 